Nonlinear Axial Stiffness Characteristics of Axisymmetric Bolted Joints

$$
\text { by }
$$

Ian R. Grosse

Dissertation submitted to the Faculty of the

Virginia Polytechnic Institute and State University

in partial fulfillment of the requirements for the degree of

Doctor of Philosophy

in

Mechanical Engineering

APPROVED:

L. D. Mitchell

C. E. Knight

N. S. Eiss

H. H. Mabie

J. N. Reddy

May 28, 1987

Blacksburg, Virginia 


\title{
Nonlinear Axial Stiffness Characteristics of Axisymmetric Bolted Joints
}

\author{
by \\ Ian R. Grosse \\ L. D. Mitchell \\ Mechanical Engineering
}

(ABSTRACT)

A critical assessment of the current design theory for bolted joints which is based on a linear, one-dimensional stiffness analysis is presented. A detailed nonlinear finite element analysis of a bolted joint conforming to ANSI standards was performed. The finite element results are presented in the classical bolted joint diagram and compared with the linear theory. The results revealed that the joint stiffness is highly dependent on the magnitude of the applied load. The joint stiffness changes continuously from extremely high for small applied loads to extremely low for large applied loads, contrary to the constant joint stiffness of the linear theory. The linear theory is shown to be extremely inadequate in characterizing the joint stiffness. The significance of the results in terms of the failure of bolted joints is discussed. Straight-forward analytical procedures are proposed for establishing estimates of the nonlinear stiffness description and the associate bolt loading in fatigue environments. The linear theory should be discarded and the more accurate nonlinear joint description be used. These results also provide the finite element community an improved model for the interconnection of substructures.

The two-dimensional, axisymmetric finite element model includes bilinear gap elements to model the interfaces. Special orthotropic elements were used to model the bolt/nut thread interaction. A free-body-diagram approach was taken by applying loads to the outer diameter of the joint model which correspond to internal, uniformly distributed 
line-shear and line-moment loads in the joint. A number of convergence studies were performed to validate the solution. 


\section{Acknowledgements}

I would like to thank my colleagues, the faculty, and the staff in the Mechanical Engineering Department for their assistance and support during my graduate years at Virginia Polytechnic Institute and State University. I would particularly like to thank the clerical/secretarial staff of Eliose Lafon, Terri Edmonson, Virginia Heck, Lynne Sawyer, Willie Hylton, Luci Zipper, and Zelda Terry for their invaluable assistance over the years with my many requests. I would like to especially thank Mr. E. L. Sittler for his continued success over the years in providing departmental financial support.

Special thanks are extended to Dr. J. B. Jones, whose encouragement and assistance five years ago brought me to graduate school. I am indebted to Dr. C. E. Knight for his advise and support over the last five years, especially during my first year. His expertise in finite elements was a source of inspiration and invaluable to me. I would like to thank Dr. N. S. Eiss, Dr. H. H. Mabie, and Dr. J. N. Reddy for their assistance also.

The last faculty member I would like to acknowledge is my friend and advisor, Dr. Larry D. Mitchell, to whom I have the highest respect and owe the deepest thanks. Larry 
never ceased to amaze me over the years with his engineering expertise, his hard work, his teaching enthusiasm, and, most importantly, his unselfish concern for his advisees. I shall strive during my academic years to emulate the standard of excellence in work and character Larry Mitchell has shown me. Patti and I are both deeply grateful to the Mitchell family for letting us house sit for them during Larry's sabbatical.

I would like to thank my family and my wife's family for their love, support, and encouragement which has guided me throughout my years in school. Finally, and without a doubt, most importantly, I extend my deepest love and thanks to my lovely and loving wife Patti. Her love and understanding, friendship and caring, has made the last five years, despite the pressures of school, the best years of my life. It is to her that I dedicate this work. 


\section{Table of Contents}

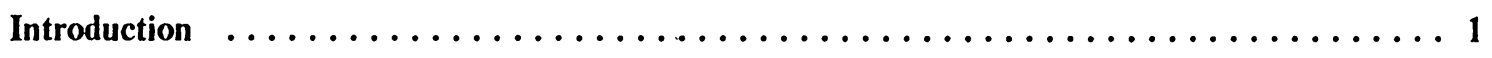

Literature Review $\ldots \ldots \ldots \ldots \ldots \ldots \ldots \ldots \ldots \ldots \ldots \ldots \ldots \ldots \ldots$

Finite Element Modeling of the Bolted Joint $\ldots \ldots \ldots \ldots \ldots \ldots \ldots \ldots \ldots \ldots \ldots \ldots \ldots \ldots$

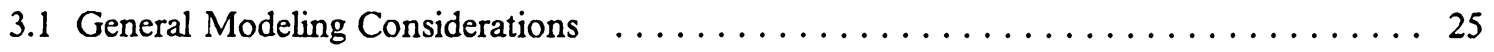

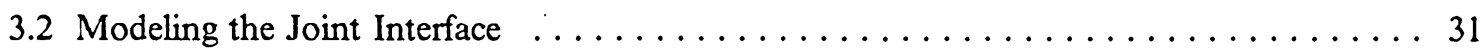

3.3 Modeling Bending in the Bolted Joint $\ldots \ldots \ldots \ldots \ldots \ldots \ldots \ldots \ldots \ldots \ldots$

3.4 Modeling the Effect of the Bolt/Nut Thread Interaction $\ldots \ldots \ldots \ldots \ldots$

3.5 Preloading the Bolted-Joint Finite Element Model $\ldots \ldots \ldots \ldots \ldots \ldots$

3.6 Loading the Bolted-Joint Finite Element Model . . . . . . . . . . . . . . 49

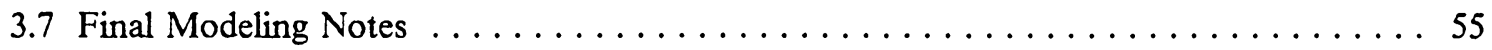

Convergence of the Bolted-Joint Finite Element Model $\ldots \ldots \ldots \ldots \ldots \ldots \ldots$

Results and Discussion $\ldots \ldots \ldots \ldots \ldots \ldots \ldots \ldots \ldots \ldots \ldots \ldots \ldots \ldots$

5.1 The Bolted-Joint under Preload $\ldots \ldots \ldots \ldots \ldots \ldots \ldots \ldots \ldots \ldots \ldots \ldots \ldots \ldots \ldots \ldots$ 
5.2 The Preloaded Bolted Joint under Applied Shear Loads $\ldots \ldots \ldots \ldots \ldots$

5.3 The Preloaded Bolted Joint under Applied Moment-Simulating Loads . . . . . . . 92

5.4 Sensitivity Studies for the Bolted-Joint Finite Element Model $\ldots \ldots \ldots \ldots \ldots$

5.4.1 The Effect of Load Step Size . . . . . . . . . . . . . . . 99

5.4.2 The Effect of the Thread Element Material Properties $\ldots \ldots \ldots \ldots \ldots \ldots \ldots 99$

5.4.3 The Effect of Interfacial Friction $\ldots \ldots \ldots \ldots \ldots \ldots \ldots \ldots \ldots \ldots \ldots \ldots \ldots \ldots$

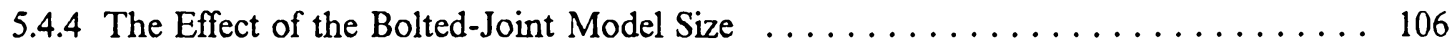

Design Application of the Nonlinear Bolted-Joint Theory . . . . . . . . . . . . . . 109

6.1 Outline For Calculating Additional Bolt Loads $\ldots \ldots \ldots \ldots \ldots \ldots \ldots$

Conclusions and Recommendations $\ldots \ldots \ldots \ldots \ldots \ldots \ldots \ldots \ldots \ldots \ldots \ldots \ldots \ldots$

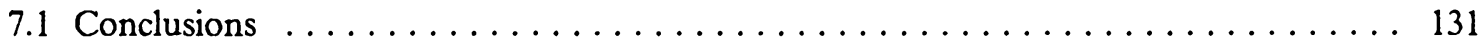

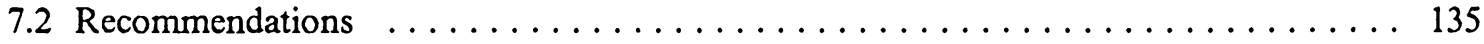

7.2.1 Recommendations for the Designer $\ldots \ldots \ldots \ldots \ldots \ldots \ldots \ldots \ldots \ldots \ldots$

7.2 .2 Recommendations for Future Research $\ldots \ldots \ldots \ldots \ldots \ldots \ldots \ldots \ldots$

References $\ldots \ldots \ldots \ldots \ldots \ldots \ldots \ldots \ldots \ldots \ldots \ldots \ldots \ldots \ldots \ldots \ldots \ldots \ldots \ldots$

Theoretical Radial Moment Equations $\ldots \ldots \ldots \ldots \ldots \ldots \ldots \ldots \ldots \ldots \ldots \ldots$

Theoretical Deflection and Rotation of Joint Member $\ldots \ldots \ldots \ldots \ldots \ldots \ldots \ldots \ldots$

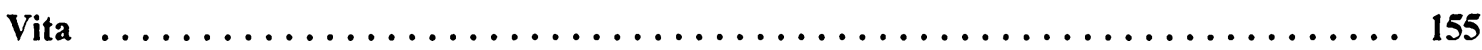

Table of Contents 


\section{List of Illustrations}

Figure 1. A Typical Bolted Joint Region $\ldots \ldots \ldots \ldots \ldots \ldots \ldots \ldots$

Figure 2. a) Experimental Setup b) Estimated Half-Face-Cone Angles (after Ito

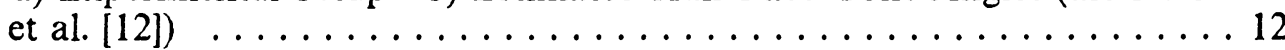

Figure 3. A Bolted-Joint Region and Spring Analogy for Intermediate Loading Planes ................................. 14

Figure 4. The Bolt-Loading Diagram $\ldots \ldots \ldots \ldots \ldots \ldots \ldots \ldots \ldots$

Figure 5. Bolted-Joint Finite Element Model of Ziada and Latif [22] ....... 18

Figure 6. Bolted Joint Configuration Tested by Chapman et al. (after Chapman et

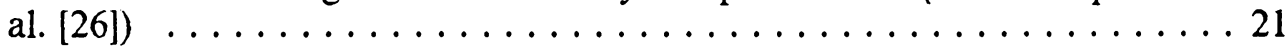

Figure 7. Experimental and Finite Element Model (after Kwiatkowski et al. [27]) 22

Figure 8. Nonlinear Bolt-Loading Diagram (after Kwiatkowski et al. [27]) . . . . 23

Figure 9. The Bolted-Joint Region Analyzed $\ldots \ldots \ldots \ldots \ldots \ldots \ldots$

Figure 10. An Axisymmetric Finite Element Model of A Bolted Joint Region . . . 29

Figure 11. Gap Element Load-Deflection Relationships $\ldots \ldots \ldots \ldots \ldots \ldots$

Figure 12. The QM4 and QM6 Elements $\ldots \ldots \ldots \ldots \ldots \ldots \ldots \ldots \ldots$

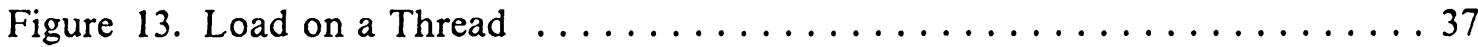

Figure 14. Bretl's FEM of Goodier's Test Specimen (after Bretl [25]) . . . . . . 40

Figure 15. This Author's FEM of Goodier's Test Specimen $\ldots \ldots \ldots \ldots \ldots 41$

Figure 16. Finite Element Deformation of Goodier's Specimen $\ldots \ldots \ldots \ldots \ldots 4$

Figure 17. Radial Expansion of the Nut (English units are used to preserve the ori-

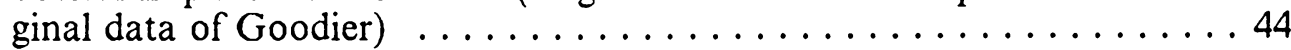


Figure 18. Illustration of Bolt Preload by Thermal-Strain Approach $\ldots \ldots \ldots 4$

Figure 19. Application of Loads to the Bolted-Joint Model. . . . . . . . . . . 50

Figure 20. FEM with Applied Stress Distribution to Simulate Positive Radial Bending Moment .......................... 52

Figure 21. a) Plate Geometry b) Radial Moment at $r=5 b$ as a function of outer plate radius a .......................... 54

Figure 22. The Bolted-Joint Finite Element Model: Convergence Study No. 1, Mesh No. $1 \ldots \ldots \ldots \ldots \ldots \ldots$

Figure 23. The Bolted-Joint Finite Element Model: Convergence Study No. 1, Mesh No. 2

Figure 24. The Bolted-Joint Finite Element Model: Convergence Study No. 1, Mesh No. 3

Figure 25. Applied Shear Load versus Bolt Strain: Convergence Study No. 1 . . . 63

Figure 26. The Bolted-Joint Finite Element Model: Convergence Study No. 2, Mesh No. 1

Figure 27. The Bolted-Joint Finite Element Model: Convergence Study No. 2, Mesh No. 2

Figure 28. The Bolted-Joint Finite Element Model: Convergence Study No. 2, Mesh No. 3

Figure 29. Applied Shear Load versus Bolt Strain: Convergence Study No. 2 . . 67

Figure 30. Zoomed Plot of Figure 29: Convergence Study No. $2 \ldots \ldots$. . . . . . 68

Figure 31. The Bolted-Joint Finite Element Model: Convergence Study No. 3, Mesh No. 1

Figure 32. The Bolted-Joint Finite Element Model: Convergence Study No. 3, Mesh

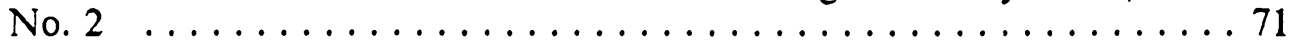

Figure 33. The Bolted-Joint Finite Element Model: Convergence Study No. 3, Mesh No. 3

Figure 34. Applied Shear Load versus Bolt Strain: Convergence Study No. 3 . . . 73

Figure 35. The Bolted-Joint Finite Element Model: Convergence Study No. 4, Mesh No. 1

Figure 36. The Bolted-Joint Finite Element Model: Convergence Study No. 4, Mesh No. 2 
Figure 37. The Bolted-Joint Finite Element Model: Convergence Study No. 4, Mesh

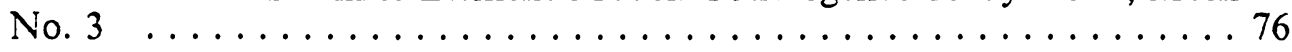

Figure 38. Applied Shear Load versus Bolt Strain: Convergence Study No. 4 . . . 77

Figure 39. Deformation of Joint Member Fixed at Inside Edge with Shear Load on Outside Edge . . . . . . . . . . . . . . . . . . . . . . . 79

Figure 40. Preload Deformation of the Bolted-Joint Model $\ldots \ldots \ldots \ldots 1$

Figure 41. Plate/Plate Interface Pressure Distribution Due to Preload . . . . . 83

Figure 42. Preload Axial Stress Distribution in the Joint Members . . . . . . . 84

Figure 43. Deformation of the Bolted Joint: $\mu=0.1, P=0.48 F_{i} \ldots \ldots 86$

Figure 44. Deformation of the Bolted Joint: $\mu=0.1, P=1.20 \mathrm{~F}_{\mathrm{i}} \ldots \ldots 87$

Figure 45. Deformation of the Bolted Joint: $\mu=0.1, P=-0.48 F_{i} \ldots \ldots 88$

Figure 46. Applied Shear Load versus Bolt Strain: $\mu=0.1 \ldots \ldots \ldots \ldots$

Figure 47. Deformation due to Applied Moment: $M=0.41 \mathrm{M}_{\mathrm{REF}} \ldots \ldots 93$

Figure 48. Deformation due to Applied Moment: $M=-0.41 M_{\text {REF }} \ldots \ldots 94$

Figure 49. Applied Moment versus Bolt Strain: $\mu=0.1 \ldots \ldots \ldots \ldots \ldots$

Figure 50. Applied Shear Load versus Bolt Strain for Combined Moment and Shear

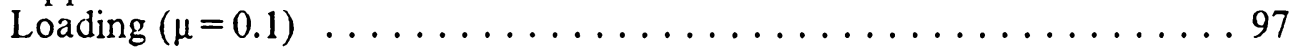

Figure 51. The Effect of Load Step Size: Applied Shear Load versus Bolt Strain

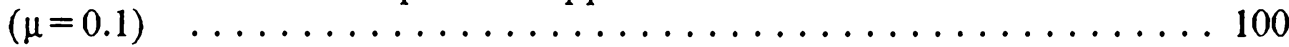

Figure 52. The Effect of the Thread Element Material Properties: Applied Shear Load versus Bolt Strain $(\mu=0.1) \quad \ldots \ldots \ldots \ldots \ldots \ldots 1$

Figure 53. The Effect of Interfacial Friction: Applied Shear Load versus Bolt Strain 104

Figure 54. The Effect of Interfacial Friction: Applied Shear Load versus Bolt Strain 105

Figure 55. The Effect of the Bolted Joint Model Size: Applied Shear Load versus Bolt Strain $(\mu=0.1) \ldots \ldots \ldots \ldots \ldots$

Figure 56. The Nonlinear Bolt Loading Diagram $(\mu=0.1) \ldots \ldots \ldots \ldots$

Figure 57. The Nonlinear Bolt Loading Diagram Relative to Preload $(\mu=0.1) \ldots 112$

Figure 58. Data Fits for Joint-Member Load Data Curve $(\mu=0.1) \ldots \ldots \ldots$

Figure 59. Data Fits for Joint-Member Load Data Curve $(\mu=0.2) \ldots \ldots$ 
Figure 60. Data Fits for Joint-Member Load Data Curve $(\mu=0.4) \ldots \ldots \ldots 119$

Figure 61. Data Fits for Joint-Member Load Data Curve $(\mu=0.8) \ldots \ldots \ldots 120$

Figure 62. The Effect of Friction on the Nonlinear Bolt Loading Diagram . . . 123 


\section{List of Tables}

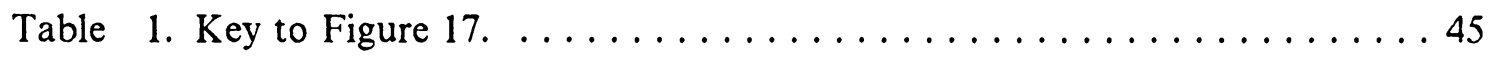

Table 2. Data Fit Coefficients of Joint-Member Load Curves . . . . . . . . 122

Table 3. Additional Bolt Loads for Various Applied Shear Loads . . . . . . . 125 


\section{Nomenclature}

\section{Roman}

$a$

$a_{i}$

A

$A_{i} \quad$ surface area associated with node $\mathrm{i}$

$A_{t} \quad$ tensile stress area of bolt

$A_{\Gamma} \quad$ circumferential area of outer joint member edge

b inner plate radius

$c \quad \cos \alpha$

C joint stiffness ratio, $=\frac{k_{b}}{k_{b}+k_{m}}$

$d$

$D_{H} \quad$ diameter of bolt thru hole

$D_{N} \quad$ diameter of nut

$E$

$F$

$F$

$F_{n}$

$F$

$F_{p}$

$F_{R}$

$F_{s}$

$F_{T}$

modulus of elasticity (subscripts denote direction)

tensile load carried by bolt

bolted-joint preload force

compressive load carried by bolt

normal interfacial force, normal thread force

proof load of bolt

resultant thread force

tangential interfacial force

tangential thread force 


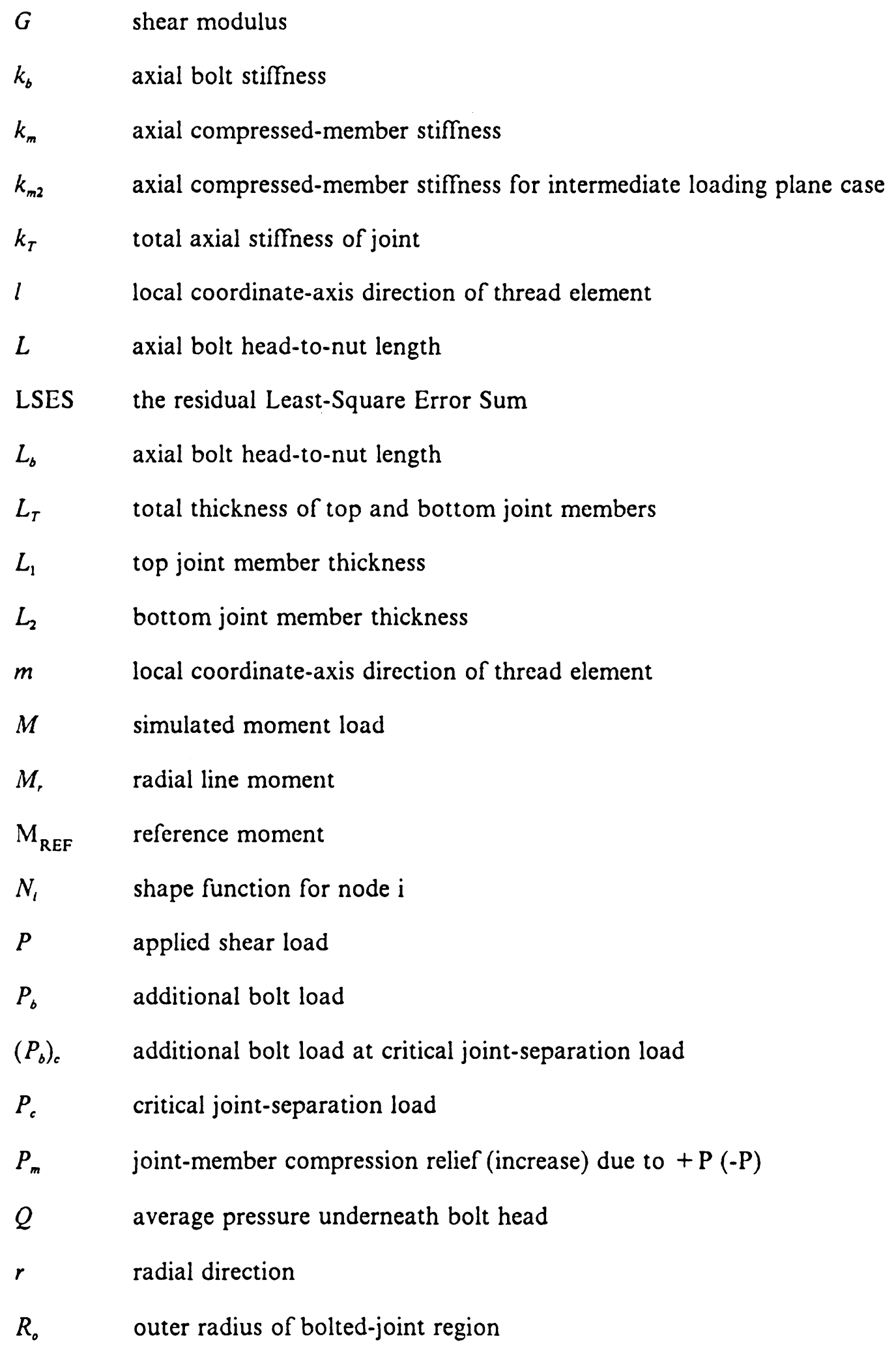


$s$

$t$

$u$

$u_{i}$

$\left(U_{s}\right)_{I} \quad$ tangential displacement of gap element's node I

$\left(U_{n}\right)_{I} \quad$ normal displacement of gap element's node I

$\boldsymbol{v}$

$v_{i}$

$x$

Greek

$\alpha$

$\sin \alpha$

plate thickness

displacement field in element $\mathrm{x}$ direction

nodal displacement in element $\mathrm{x}$ direction

displacement field in element y direction

nodal displacement in element y direction analysis

global coordinate-axis direction- the bolt-axial direction axisymmetric analysis

angle of inclination of special thread elements

axial coefficient of thermal expansion

Unified half-thread angle $=30^{\circ}$

half-face-cone angle of conical frustrum

axial bolt extension or contraction

denotes increment of variable that follows

change in bolt length relative to preload bolt length

average axial bolt strain relative to preload bolt strain

global coordinate-axis direction- radial direction for axisymmetric

global coordinate-axis direction- the hoop-stress direction for

average axial bolt strain relative to preload bolt strain at the 
critical joint-separation load

$\varepsilon$ average bolt strain

$\varepsilon_{i} \quad$ bolt preload strain

intermediate loading plane factor local element coordinate-axis direction

$\theta$

$\lambda \quad=\frac{\Delta \varepsilon}{\Delta \varepsilon_{c}}$

$\mu$

interfacial coefficient of friction

$v$

Poisson's ratio

$\xi \quad$ local element coordinate-axis direction

$\sigma$

normal stress

$\sigma_{i}$

axial bolt stress due to preload $=\frac{F_{i}}{A_{t}}$

$\tau$

shear stress

Subscripts Frequently Used

b denotes bolt-related variable

c

denotes critical joint-separation related variable

i

denotes either nodal variable or preload-related variable

m

denotes joint-member-related variable

r

denotes radial direction

$\mathrm{x}$

denotes $\mathrm{x}$ or radial direction

$\mathrm{y}$

denotes axial direction

$\theta$

denotes hoop-stress direction 


\section{Chapter 1}

\section{Introduction}

Bolted joints often play a critical role in mechanical systems. Failure of a joint can cause catastrophic failure of a structure or machine, such as the collapse of the skywalk in the St. Louis Hyatt Regency which resulted in a number of fatalities. Another example is the collapse of a ten-ton overhead monorail electric hoist used in a rolling mill which killed the operator [1]. ${ }^{1}$ In addition, in many cases the stiffnesses of the bolted-joint connections within a system play a large role in influencing the operating behavior of the system as a whole. For example, the fundamental vibration frequency of a machine bolted to a foundation is highly dependent on the stiffnesses of the bolt/foundation joint regions. Yet, analysis of a bolted-joint region is quite difficult in general. Current analytical methods of analyzing bolted-joint regions consist of invoking simplifying assumptions of either assuming a rigid joint connection (i.e. no joint) or the use of simplified linear models.

1 Numbers in brackets refer to References at end of this dissertation. 
However, joint regions are highly nonlinear systems. This is not generally recognized by the structural analysis community. The nonlinearity of jointed connections is due to the fact that the stiffnesses of the joint members in compression are proportional to the interface contact area between the joint members. The interface contact area depends on the joint preload and on the external loads carried by the joint. Consequently, joint member stiffnesses and the overall joint region stiffness are functions of the external loads carried by the joint.

There are several other factors contributing to the difficulty of developing analytical joint models. Experimental data must be available to accurately predict the shape and size of the compressed member region which is critical in determining the joint stiffness. The geometric complexities of the bolted-joint region, combined with deformations due to preload, make closed-form analytical solutions of the governing equations of elasticity virtually impossible. In particular, the geometry of the bolt and nut threading and their interaction are too complicated to handle analytically. The problem is further complicated by the existence of friction between the bolt-nut threads, between the bolt head and the compressed joint member, between the nut and the other compressed joint member, and between the joint members themselves along the joint interface.

In light of the above discussion, accurate models of bolted-joint regions can only be obtained computationally or experimentally. It is the objective of this dissertation to determine the axial stiffness of bolted-joint regions through finite element analysis and to incorporate these results into a new design methodology. The new design methodology will enable the designer to more accurately calculate the loads that are carried by the bolt and thereby improve the design of bolted joints. 
Finite element analysis of bolted-joint regions, although complicated in itself, is considerably quicker and more versatile than experimentation. Furthermore, existing experimental data can be incorporated into the analysis to improve the accuracy of the model.

The nonlinearity of the bolted-joint region means an iterative finite element analysis is needed. Since the computer time required for each iteration is roughly proportional to the square of the mean bandwidth of the structure stiffness matrix, it is important to keep the stiffness matrix of the joint region as banded as possible. In general, threedimensional finite element models of solids have associated structure stiffness matrices with large bandwidths. Hence, an important assumption in this work is the axisymmetric assumption which allows modeling of the solid joint region with a detailed two-dimensional finite element model with a relatively small bandwidth on the stiffness matrix. A corresponding three-dimensional finite element model with the same number degrees of freedom would have a much coarser mesh and a much larger bandwidth on the stiffness matrix.

The axisymmetric assumption, however, introduces some approximations and restrictions into the finite element analysis. The obvious geometric approximations are that both the bolt head and nut are cornerless. Another geometric approximation due to the assumption of axisymmetry is the neglecting of the thread helix angle inherent in the special axisymmetric thread elements used here. The most important point to note, though, is that axisymmetric, nonlinear finite element models of the bolted-joint region are restricted to axisymmetric loading. Usually, nonaxisymmetric loading conditions can be handled in an axisymmetric finite element model by expanding the nonaxisymmetric load distribution as a finite Fourier series in terms of the angle $\theta$. Each term of the series yields a separate solution which are superimposed to yield the solution to the 
nonaxisymmetric load. However, the principle of superposition is not valid for nonlinear systems. Hence, nonaxisymmetric loading conditions for the bolted-joint region can only be dealt with in a general three-dimensional finite element analysis which is beyond the scope of this work. Yet, there are many applications in which loads on a bolted joint can be approximated as axisymmetric. For example, bolt loads in bolted connections of structures connected to base plates undergoing vertical motion can be approximated as axisymmetric in the absence of rocking motion. Also, loads carried by individual bolts within bolt patterns of tensile bolted connections may be approximated as axisymmetric. In general, the more bolts used in a bolted connection, the more realistic it is to assume that loads transmitted to an individual bolted-joint region are axisymmetric.

It is hoped that this work will provide the first important step in the development of a bolted-joint region finite element. In general, this element will be nonlinear, but it could be linearized about known operating points. Such an element would allow finite element analysts to model an entire bolted-joint region within a structure with a single finite element. The alternative is to discretize the bolted-joint regions within a structure by hundreds of finite elements. If the structure has more than just a few bolted connections, such an approach would exceed the computational resources presently available. As more work is done in this area, it is conceivable that a library of bolted-joint region elements can be developed corresponding to bolt and nut configurations governed by ANSI standards. The finite element analyst could then select the appropriate joint elements from the element library during the model creation stage. 
The finite element analyses presented in this dissertation were carried out an IBM 4341-2 computer using ANSYS ${ }^{\circledR}, 2$ a proprietary engineering analysis computer program developed by Swanson Analysis Systems, Incorporated. The author has assumed that the reader has a basic familiarity with the finite element method. If not, the reader may wish to consult some of the many textbooks on the subject, such as texts by Cook [2], Reddy [3], Segerlind [4], Bathe and Wilson [5] and Zienkiewicz [6]. Some theoretical background will be given as needed for specific topics in finite element modeling relevant to the bolted-joint problem and the use of ANSYS. Cook's finite element notation and bolted-joint design notation of Shigley and Mitchell [7] have been adopted.

2 ANSYS is a registered trademark of Swanson Analysis Systems, Inc.. The notation ANSYS will be used for all subsequent references. 


\section{Chapter 2}

\section{Literature Review}

A typical bolted-joint region is shown in Figure 1. The $\mathrm{x}$ direction is the horizontal direction, the radial direction in an axisymmetric analysis. The y direction is taken as the axial direction of the bolt. For an axisymmetric analysis, the $\mathrm{z}$ direction is the $\theta$ or hoop stress direction.

The classical approach for obtaining the axial joint stiffness region is based on a onedimensional spring analogy. When the external load is applied as shown in Figure 1, the spring analogy has the bolt axial stiffness between the bolt head and nut acting in parallel with the axial stiffness of the compressed members between the the bolt head and nut. The external load $\mathrm{P}$ is then divided into relieving the bolted-member's compression and increasing the bolt's tensile load according to the relative stiffnesses of the bolted member and the bolt 


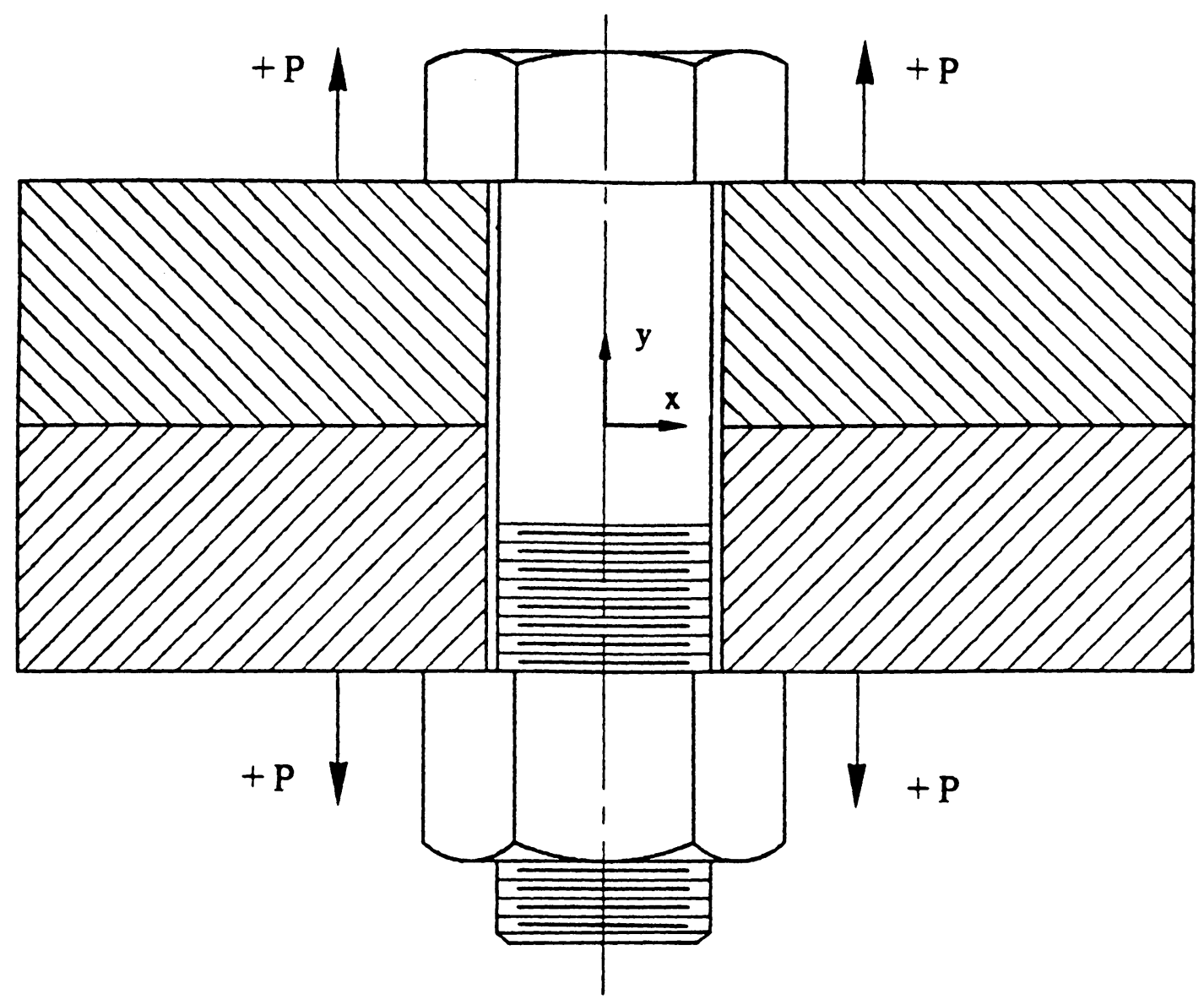

Figure 1. A Typical Bolted Joint Region 


$$
\begin{array}{rlrl}
P & =P_{b}+P_{m} & & \\
P_{b} & =C P, & & 0 \leq P \leq P_{c} \\
P_{m} & =(1-C) P, & & 0 \leq P \leq P_{c} \\
P_{b} & =P & & P>P_{c} \\
P_{m} & =0 & P & >P_{c}
\end{array}
$$

where $P_{c}$ is the critical load that causes separation and $\mathrm{C}$ is the the joint stiffness ratio defined by

$$
C \equiv \frac{k_{b}}{k_{b}+k_{m}}
$$

The total axial joint stiffness, $k_{T}$, is the sum of the bolt and the compressed-member stiffnesses

$$
k_{T}=k_{b}+k_{m}
$$

The tensile load carried by the bolt, $F_{b}$, and the total compressive load carried by the joint members, $F_{m}$, are

$$
\begin{gathered}
F_{b}=F_{i}+P_{b} \\
F_{m}=F_{i}-P_{m}
\end{gathered}
$$

$P_{c}$ occurs when $F_{m}=0$ and is easily obtained from Eqs. (2.4) and (2.1) as

$$
P_{c}=\frac{F_{i}}{(1-C)}
$$


Ideally, one would like to reduce the portion of the external load carried by the bolt since both static and fatigue failures generally occur in the bolt. From Eqs. (2.1) and (2.2), one notes that if $k_{m}>>k_{b}$, then $P_{b}$ approaches zero. Hence, it is advantageous to use stiff joint members with a relatively flexible bolt to prevent bolt failure.

The load equations presented above contain only two parameters that need to be determined: the axial stiffness of the bolt, $k_{b}$, and the axial compressed-member stiffness, $k_{m}$. The axial stiffness of the bolt, $k_{b}$, is easily obtained.

$$
k_{b}=\frac{E}{\sum_{i=1}^{n} \frac{l_{i}}{A_{i}}}
$$

where $\mathrm{n}$ is the number of sections of the bolt with different effective diameters and where $A_{i}$ and $l_{i}$ are the sectional area and length of the $\mathrm{i}^{\text {th }}$ section. The thread tensile stress area is used to obtain the threaded section's effective diameter. For short bolts (usually categorized as having a grip length less than three shank diameters), analysts ([8],[9]) recommend that the elasticity of the bolt head and nut be considered in determining $k_{b}$. Bickford [8] recommends increasing the length of sections $l_{i}$ in Eq. (2.6) adjacent to the bolt head and nut by one half the bolt head and nut heights, respectively.

The difficulty with the classical approach lies in determining $k_{m}$. To obtain this stiffness, analysts generally make the following approximations:

- assume a volumetric shape of the compressed region of the joint members

- assume that $\sigma_{y}$ is uniform across any horizontal plane within the compressed region

- assume other stress components are small compared to $\sigma_{y}$

- assume the shape of the compression region is not affected by external loads 
With these assumptions one can then integrate the the differential strain $d \varepsilon_{y}$ due to an axial unit load through the thickness of the joint members. Inverting the result yields the stiffness of the compressed-member stiffness.

The one-dimensional spring analogy was first applied by Rotscher [10] in 1927. Rotscher assumed a double-conical-frustrum shape for the compressed region of the joint members. He then replaced the frustrum shape with an equivalent cylinder of equal sectional area and calculated the stiffness of the joint member. Birger [11] integrated directly the differential strain $d \varepsilon_{y}$ for Rotscher's model to obtain the well-known formula for the stiffness of the compressed region for the case of two joint members of equal thickness

$$
k_{m}=\frac{\pi D_{H} E \tan \gamma}{2 \ln \left[\frac{\left(D_{N}+L_{T} \tan \gamma-D_{H}\right)}{\left(D_{N}+L_{T} \tan \gamma+D_{H}\right)} \frac{\left(D_{N}+D_{H}\right)}{\left(D_{N}-D_{H}\right)}\right]}
$$

Birger also used a single frustrum model for the case of a single joint member bolted to a foundation with a blind hole. Neglecting the elasticity of the blind-hole member, Birger obtained the single member stiffness as

$$
k_{m}=\frac{\pi D_{H} E \tan \gamma}{\ln \left[\frac{\left(D_{N}+2 L \tan \gamma-D_{H}\right)}{\left(D_{N}+2 L \tan \gamma+D_{H}\right)} \frac{\left(D_{N}+D_{H}\right)}{\left(D_{N}-D_{H}\right)}\right]}
$$

where $L$ is the thickness of the single joint member considered here.

In both Eqs. (2.7) and (2.8) the stiffness of the joint members is a nonlinear function of $\gamma$, the half-face-cone angle of the frustrum or double frustrum model. Various values of $\gamma$ have been proposed, ranging from 20 degrees to 45 degrees. Ito et al. [12] experimentally measured the interface pressure distributions and interface contact areas for 
various single bolted-joint proportions. The authors used the measured outer diameter of the interface contact area to obtain an estimate of the half-face-cone angle $\gamma$ used in the above theory. Figure 2 shows the authors experimental setup and the half-face-cone angles obtained for four flange thicknesses and for three flange materials- semi-hard steel, brass, and an aluminum alloy.

Other shapes have been proposed to model the compressed joint area. Samonov [13] introduced both a single and double paraboloid of revolution to model the compression region for the blind-hole problem and the two joint member problem. He then integrated the differential strain to obtain similar formulas for the compressed member stiffnesses. Samonov's approach required an assumed maximum diameter of the paraboloid.

Motosh [14] presents an excellent summary of the joint member stiffnesses obtained by using a uniform $\sigma_{y}$ stress distribution assumption. Motosh then improved the classical theory by introducing a polynomial representation of $\sigma_{y}$ as

$$
\sigma_{y}=A r^{4}+B r^{3}+C^{r} 2+D r+E
$$

where the coefficients $\mathrm{A}, \mathrm{B}, \mathrm{C}, \mathrm{D}$, and $\mathrm{E}$ are functions of $\mathrm{y}$. Thus, $\sigma_{y}$ is a function of both the joint radius, $r$, and the axial direction, $y$. Motosh determined the coefficients by assuming boundary conditions on the stress distribution that agreed with experimentally observed stress distributions. However, he still assumed that the other stress components were small compared to $\sigma_{y}$. Nethertheless, his results compared favorably with experimental data taken by Fritsche [15]. 


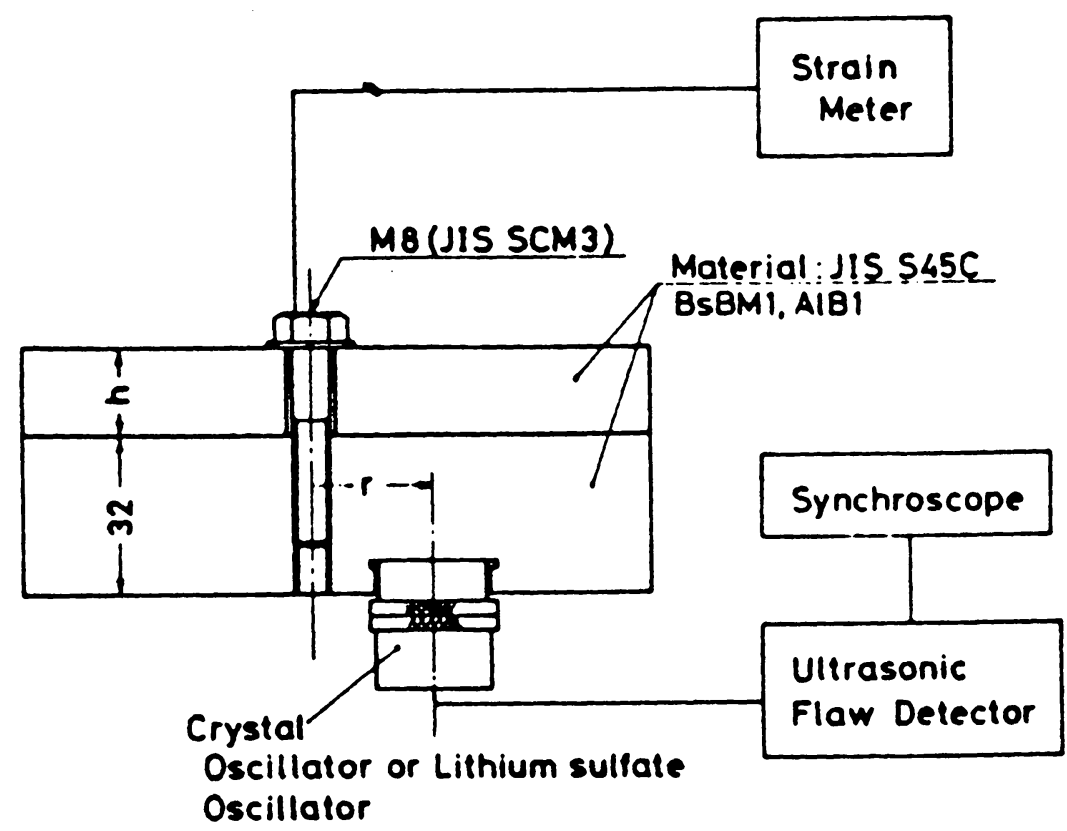

Fig. 1 Scheme of the apparatus

a)

Table 2 Example of measured values of the half angle of pressure cone (ground joint surfaces)

\begin{tabular}{|c|c|c|c|}
\hline \multirow{2}{*}{$n \mathrm{~mm}$} & \multicolumn{3}{|c|}{$\alpha$ deg } \\
\cline { 2 - 4 } & \multicolumn{3}{|c|}{ Flange material } \\
\cline { 2 - 4 } & $S 45 C$ & $B_{5} B M 1$ & $A 1 B 1$ \\
\hline 8 & 55 & 73 & 73 \\
\hline 16 & 36 & 59 & 59 \\
\hline 24 & 30 & 47 & 47 \\
\hline 32 & 24 & 39 & 39 \\
\hline
\end{tabular}

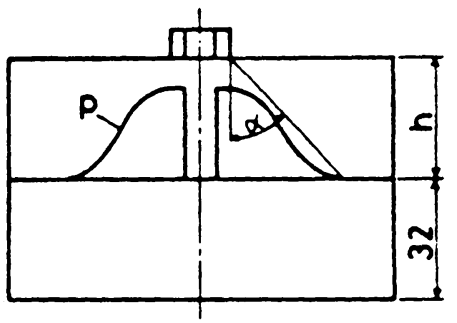

b)

Figure 2. a) Experimental Setup b) Estimated Half-Face-Cone Angles (after Ito et al. [12]) 
Samonov [13] was the first to modify the conventional theory to account for the influence of the location of the external load has on the joint member stiffness. He used a more complicated spring analogy to derive the bolt load and joint member load equations for the blind-hole member problem. Sharp [9] extended this analogy to the case of two joint members bolted together. As seen in Figure 3, the external load is assumed to be applied on some horizontal loading plane a distance $d$ from the interfaces of the joint members. Using the notation of Figure 3 and the spring analogy, the following load equations can be easily derived,

$$
\begin{aligned}
P_{b} & =\zeta C P \\
P_{m} & =(1-\zeta C) P \\
P_{c} & =\frac{F_{i}}{(1-\zeta C)}
\end{aligned}
$$

where $\mathrm{C}$ is as defined in Eq. (2.2) and $\zeta=k_{m} / k_{m 2}$. Note that $\zeta=1$ corresponds to loading under the bolt head and nut (see Figure 1 on page 7), and $\zeta=0$ corresponds to the axial loading applied directly at the joint interface. It can also be seen from Figure 3 and Eq. (2.10) that applying the axial load directly to the interface results in all of the external load being absorbed by compression relief in the joint member. None of the external load is transferred to the bolt. Thus, until the compression is removed in the joint, this theory states that the joint is infinitely stiff.

It is generally assumed that $\zeta$ can be approximated by $d / L$, where $d$ is the distance from the joint interface to the horizontal loading plane and $L$ is the thickness of one of the joint members. However, since loads generally do not enter a bolted-joint region on a horizontal plane, the location of this hypothetical loading plane is not known apriori. Bickford [8] claims that computations based on assuming values for $P_{b}, P_{m}$ and $P_{c}$ half- 


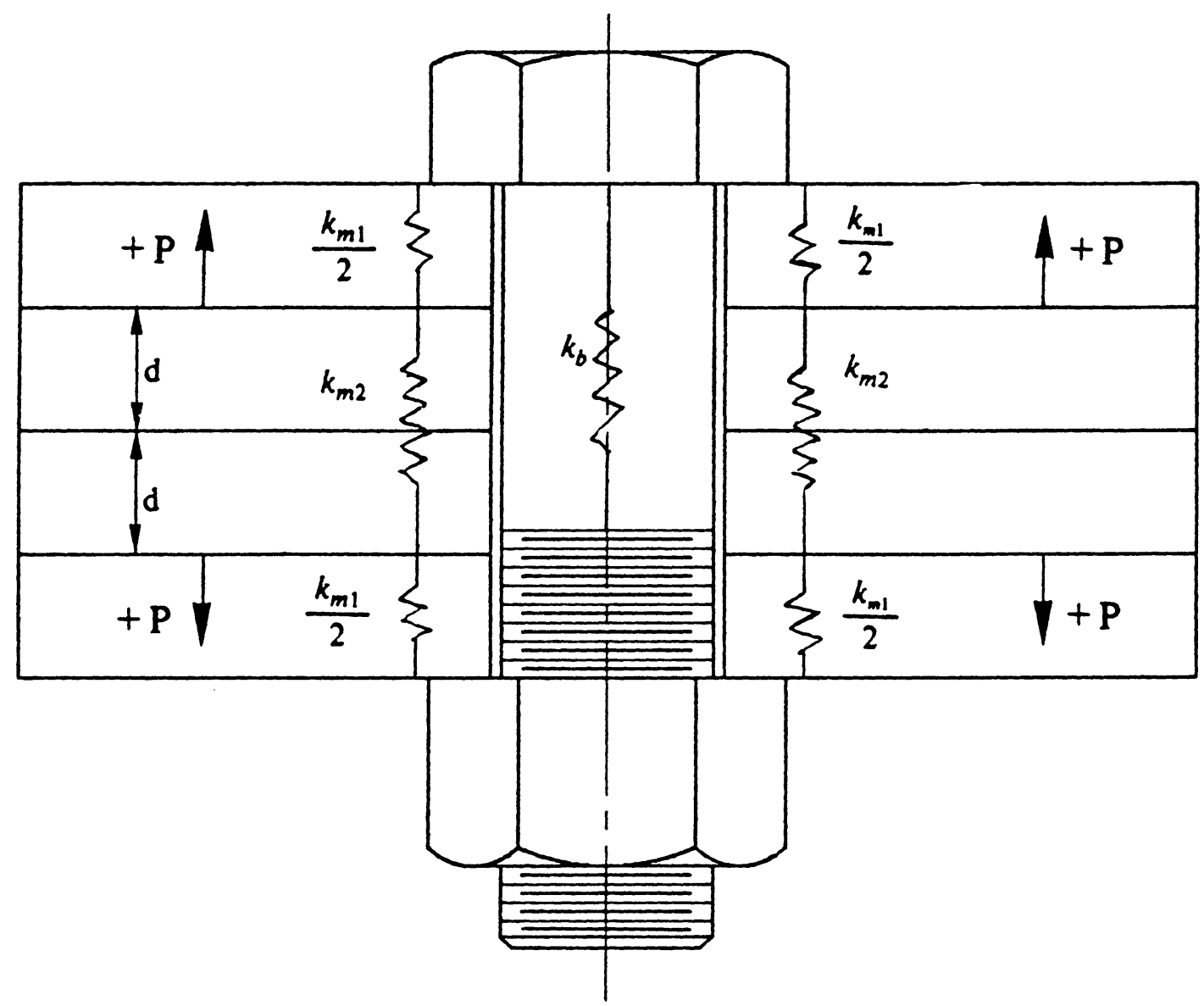

Figure 3. A Bolted Joint Region and Spring Analogy for Intcrmediate Loading Planes 
way between the values obtained for $\zeta=0$ and $\zeta=1$ "will be accurate enough for virtually all applications."

The above theory has been incorporated into the popular "Bolt-Loading Diagram" shown in Figure 4 by Radzimovsky [16], Phelan [17], Faires [18], Samonov [13], Meyer and Strelow [19], and others. The diagram provides a graphical approach for calculating the loads carried by the bolt and the joint member. The dashed load lines adjacent to the solid bolt and joint-member load lines are the modified load lines proposed by Samonov [13] and Meyer and Strelow [19] for the case of an intermediate loading plane. To use the diagram, the external tensile load $\mathrm{P}$ is scaled as a vector using the ordinate scale. This vector is then fitted vertically between the bolt load curve and the joint member load curve as shown. One can then read directly from the ordinate scale the tensile load carried by the bolt and the compressive load carried by the joint members. Also shown in Figure 4 are the time-varying loads in the bolt and compressed member if the external load $\mathrm{P}$ were a sinusoidal time-varying load with mean value of $+\mathrm{P} / 2$ and alternating component of $\mathrm{P} / 2$. It is easy to see from the diagram that a steeper joint member load curve (i.e. stiffer joint members) results in a smaller portion of the external load taken by the bolt.

In addition to the classical approach to analyzing bolted joints, finite element analysis has also been used to study the bolted-joint problem. Gould and Mikic [20] modeled the compressed member region of a bolted joint using axisymmetric, linear elastic plates with a through hole. The bolt and nut were excluded from the mathematical model. Compression in the plates was introduced by applied pressure distributions on the surfaces of the plates corresponding to the locations of the bolt head and nut. The authors determined pressure distributions and the radii at which the plates separate for different 

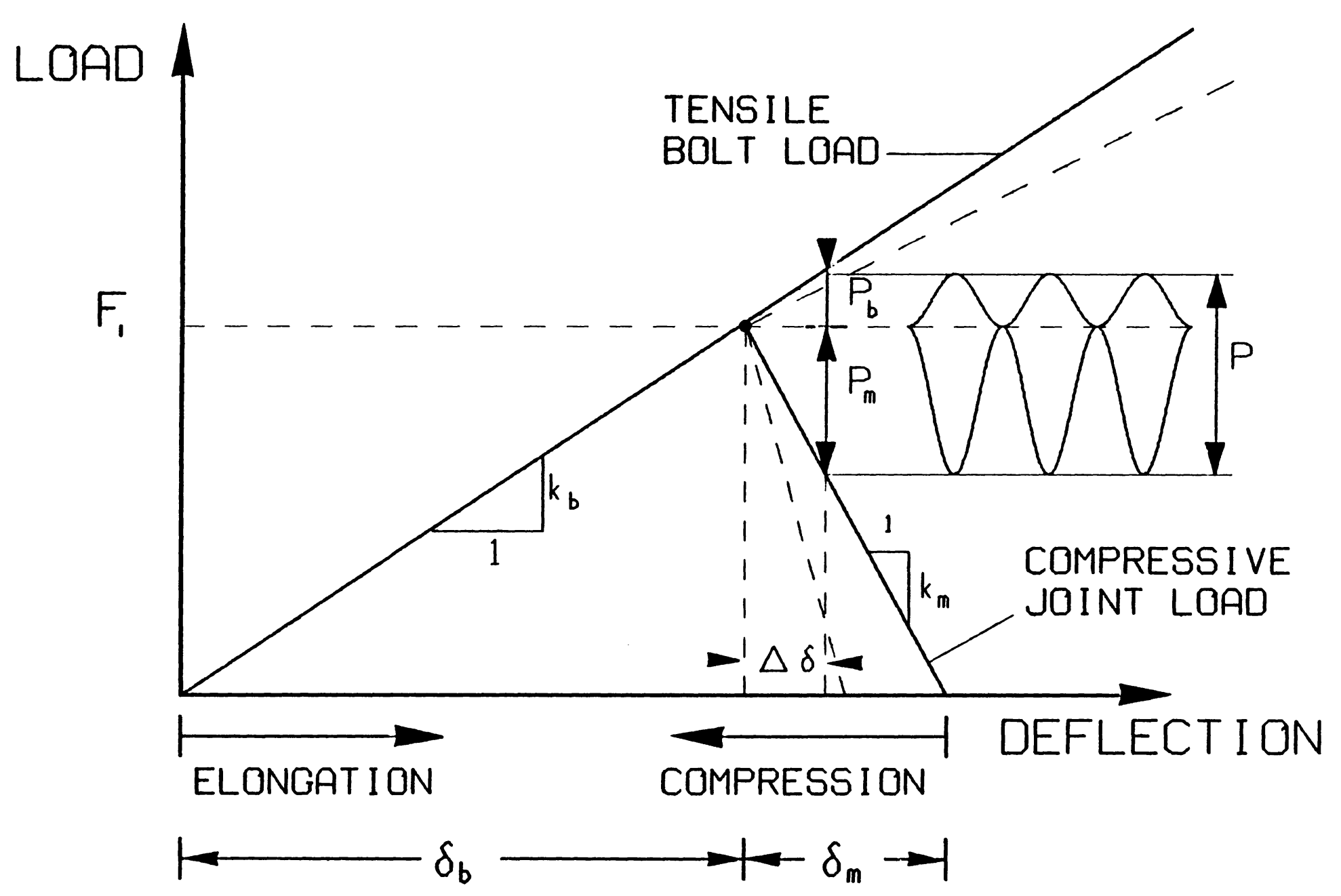
plate thickness ratios, $\frac{L_{1}}{L_{2}}$. This model did not allow any sliding at the joint interface. Maruyama et al. [21] applied the finite element technique to the bolted joint to obtain the pressure distribution on the bolt head bearing surface member interface and to obtain the axial joint stiffness under preload. The bolt head, a portion of the bolt shank, and a single joint member were modeled with two-dimensional, axisymmetric triangular elements. Friction at the bolt head/plate interface was disregarded.

Ziada and Latif [22] modeled both joint (plate) members and the bolt and nut with two-dimensional, axisymmetric finite element models. Figure 5 shows one of their models. The plate/plate, bolt head/plate, and nut/plate interfaces were modeled with two-dimensional gap elements which may maintain or break physical contact and slide relative to each other. An unusually high coefficient of friction of 0.75 was used for the interface elements. The bolt preload was developed by introducing axial displacements at the bolt end. No external loads were applied to the model The authors studied the effect of the plate thickness ratio on the joint loads and stress distribution. Later, Ziada and Latif ([23], [24]) studied the effect of the outer joint diameter on the load and stress distribution. They also calculated the joint stiffness from the finite element model deformations. However, they modeled the bolt and nut as a single structural member as if the nut were rigidly fixed to the bolt. No attempt was made to model the effect of the bolt/nut threading interaction. In addition, the authors did not address the basic nonlinearity of the bolted-joint system; i.e., how external axial loads influence the joint stiffness.

Bretl [25] studied the interaction of the bolt/nut threading and introduced special axisymmetric elements to model its effect. A modified version of these elements were incorporated into the finite element models used here. Therefore, Betl's work will be 


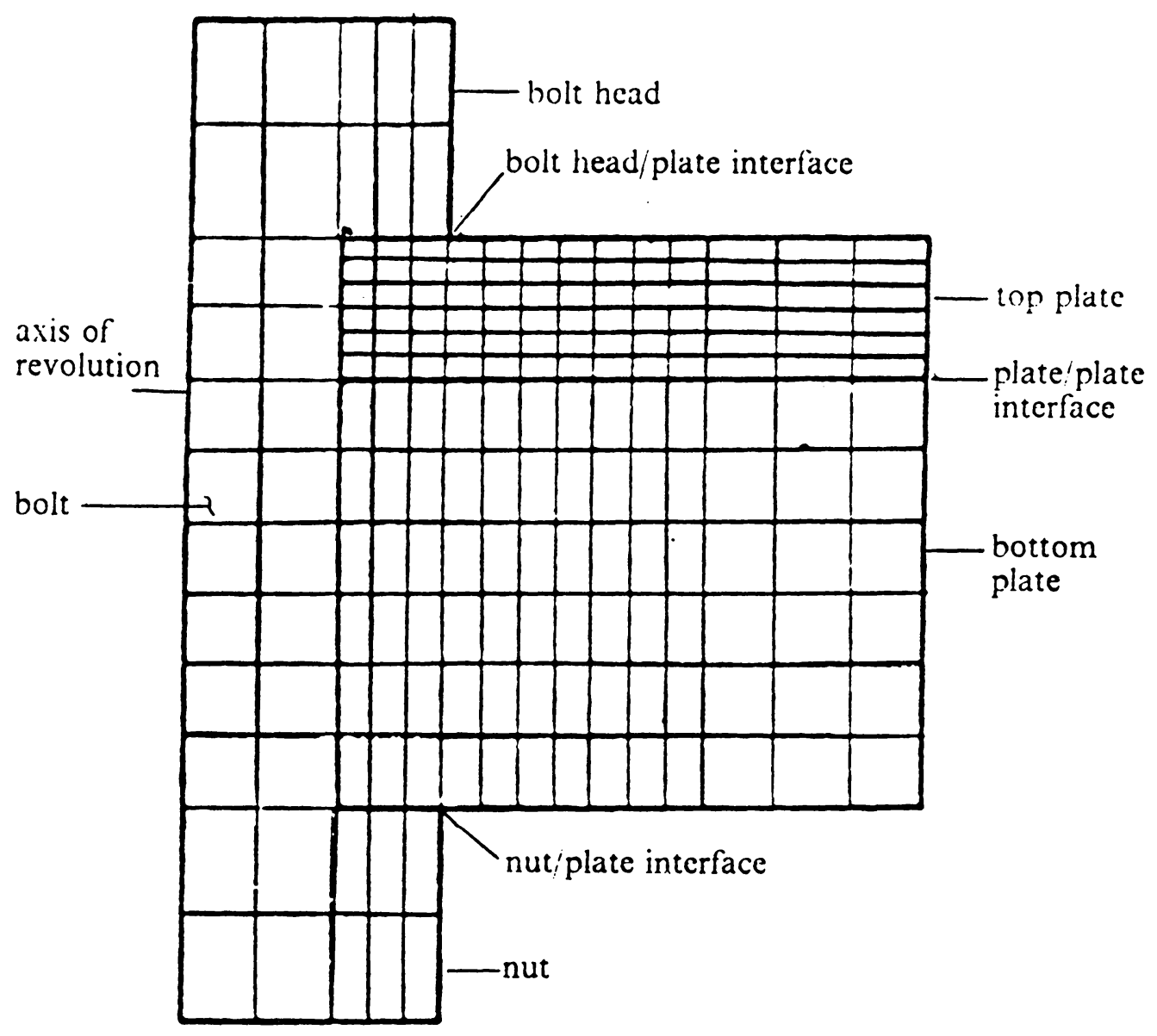

Figure 5. Bolted-Joint Finite Element Model of Ziada and Latif [22] 
addressed in detail in Chapter 3. These "thread" elements are an important contibution to the modeling of bolted-joint regions. They model the effect of the thread interaction without modeling the detailed thread profile. Modeling the thread geometry, as well as the remaining bolted-joint region, would result in an extremely large finite element model. Of course, by modeling the effect and not the thread geometry itself, one can not obtain accurate stress information in the thread region. But this does not present a problem since our principle objective is to characterize the stiffness of the bolted-joint region.

In the above references the basic nonlinearity of the bolted-joint problem- the fact that the stiffness of the compressed joint member is dependent on the external load- was not considered. In the classical approach this linearization of the problem is evident in the constant-slope joint member load curves of Figure 4 on page 16. The slope represents the stiffness of the compressed joint members $k_{m}$. In addition, the classical approach does not consider the possibility that compressive external loads, instead of tensile external loads, may be applied to the joint in practice. Meyer and Strelow [19] point out that for the purposes of design, the classical bolted-joint diagram should be modified to reflect the change in the joint member stiffness as external loads are applied. The authors note that the stiffness of the joint varies for both tensile and compressive applied loads and, in fact, present on a classical bolted-joint diagram a nonlinear joint member load curve for both tensile and compressive applied loads. The curve does not appear to be based on any data or calculations but rather on intuition. The authors point to the need for further work in this area to expand on this concept.

Chapman et al. [26] empirically determined the response of a bolt to axial external loading after tightening. The authors tested a metric grade 12.9 bolt with a minimum 
tensile yield stress and load of $1080 \mathrm{MPa}$ and $62500 \mathrm{~N}$, respectively. Figure 6 shows the joint configuration tested by the authors. The external tensile load was applied to the joint through tensile rods threaded into each plate. If one believes Reimer et al.'s nonlinear bolted-member stiffness, one is surprised that the authors report a linear relationship between the portion of the external load carried by the bolt and the external load. One would expect the fraction of the external load carried by the bolt to increase with external load as the joint separates.

Recently, important experimental and finite element data has been published by Kwiatkowski et al. [27] which, in fact, reveal a highly nonlinear bolted-joint system. Figure 7 shows a sketch of their experimental model. The bolt was preloaded to approximately $90 \%$ of its proof load and an external tensile load was incrementally applied until the joint separated and then unloaded incrementally. Figure 7 also shows a sectional view of their three-dimensional finite element model. The model included only the upper half of the joint by assuming symmetry about the joint member interface plane. The effect of the bolt/nut thread interaction is not included in their work. In addition, the symmetry assumption about the joint interface plane ignores the significant geometric differences between the bolt head and nut. The authors took into account the elastic-plastic material properties using a visco-plastic formulation. Fictitious contact layers were used to model the friction effects and possible slips between joint elements. The authors present a nonlinear bolt loading diagram shown in Figure 8.

The literature on finite element modeling of bolted joint reveals three major shortcomings. First, analysts have neglected to include the effect of the bolt/nut thread interaction. This is surprising since Bretl's special "thread" elements were introduced nine years ago. Secondly, most of the work has been devoted to obtaining the deformation 


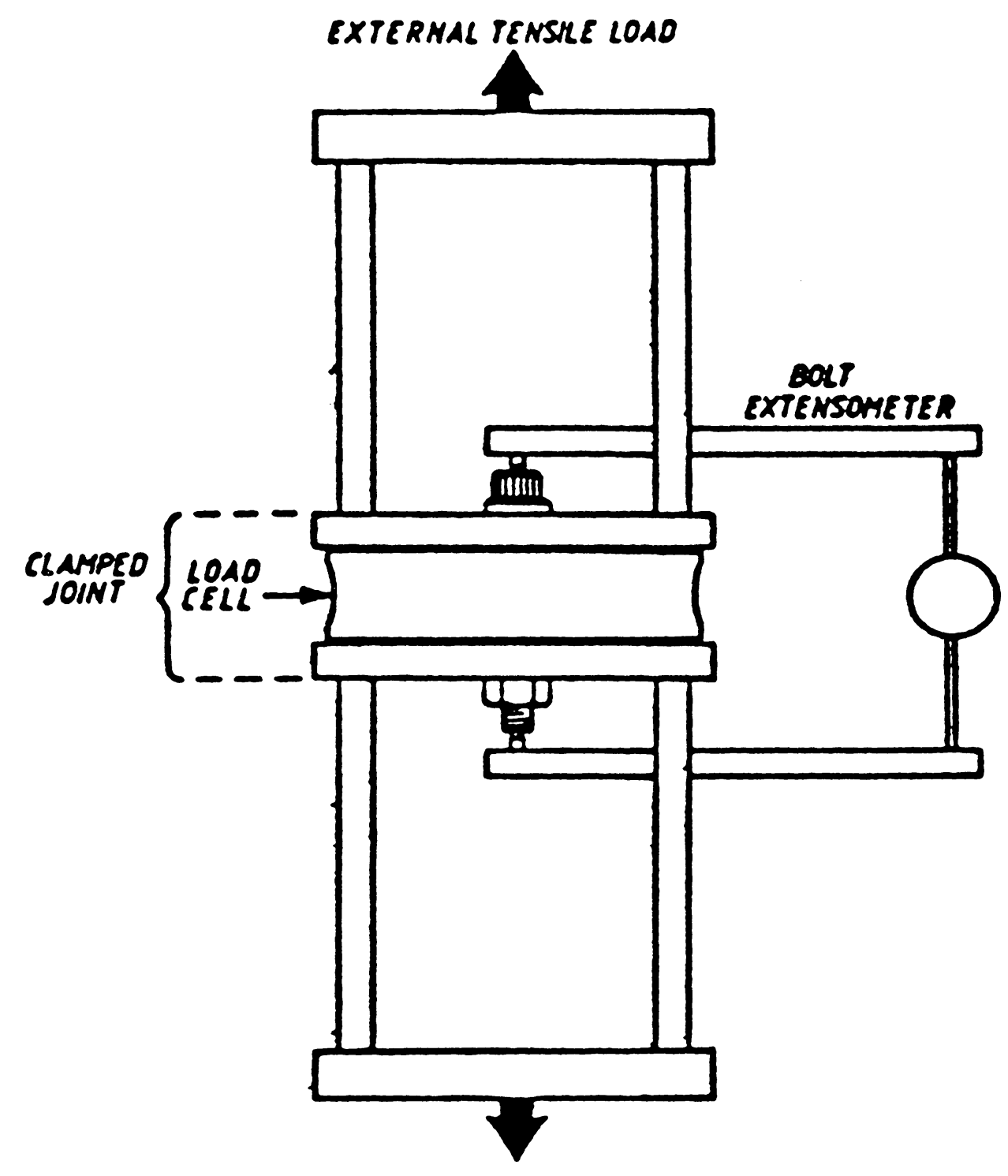

Figure 6. Bolted Joint Configuration Tested by Chapman et al. (after Chapman et al. [26]) 

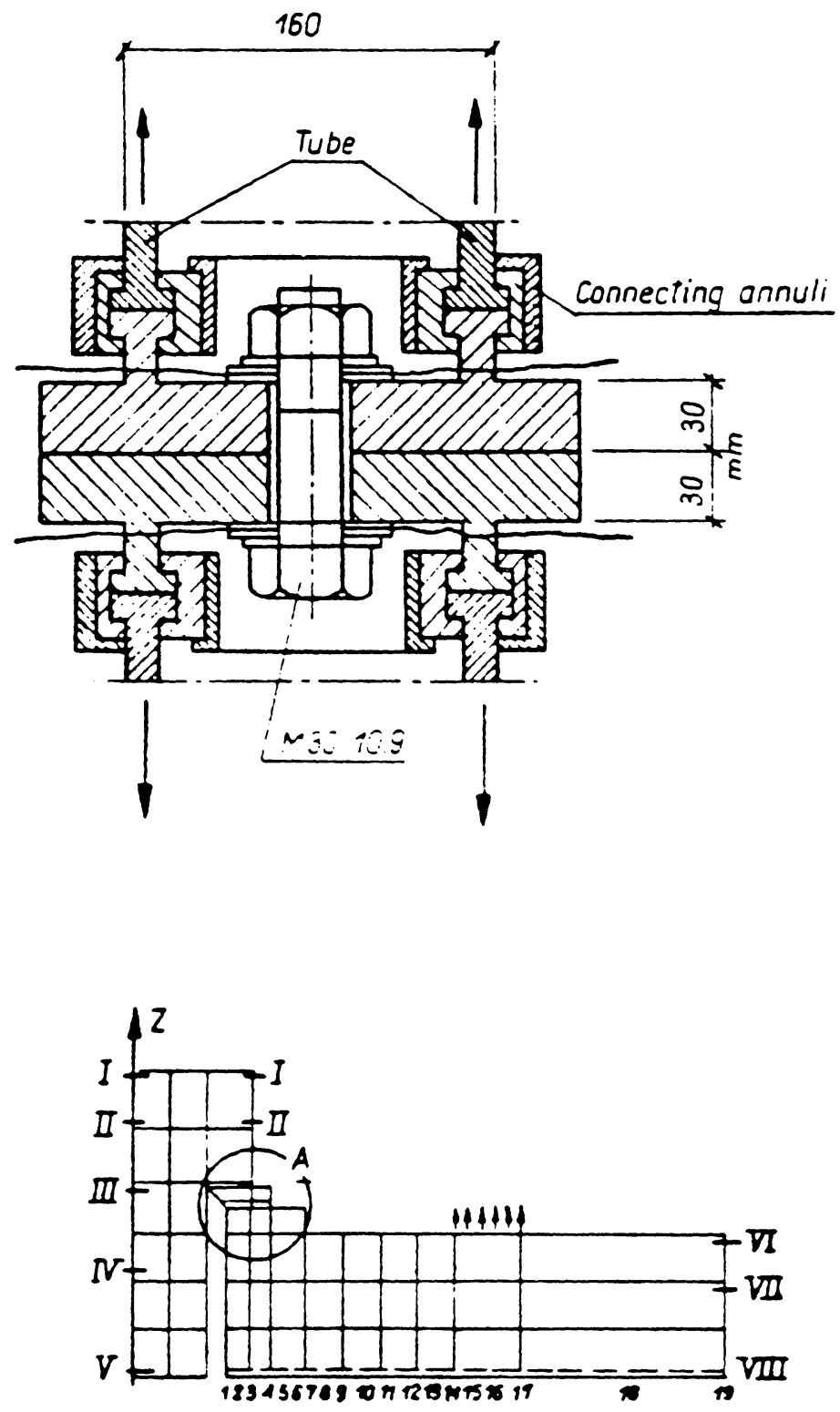

Figure 7. Experimental and Finite Element Model (after Kwiatkowski et al. [27]) 


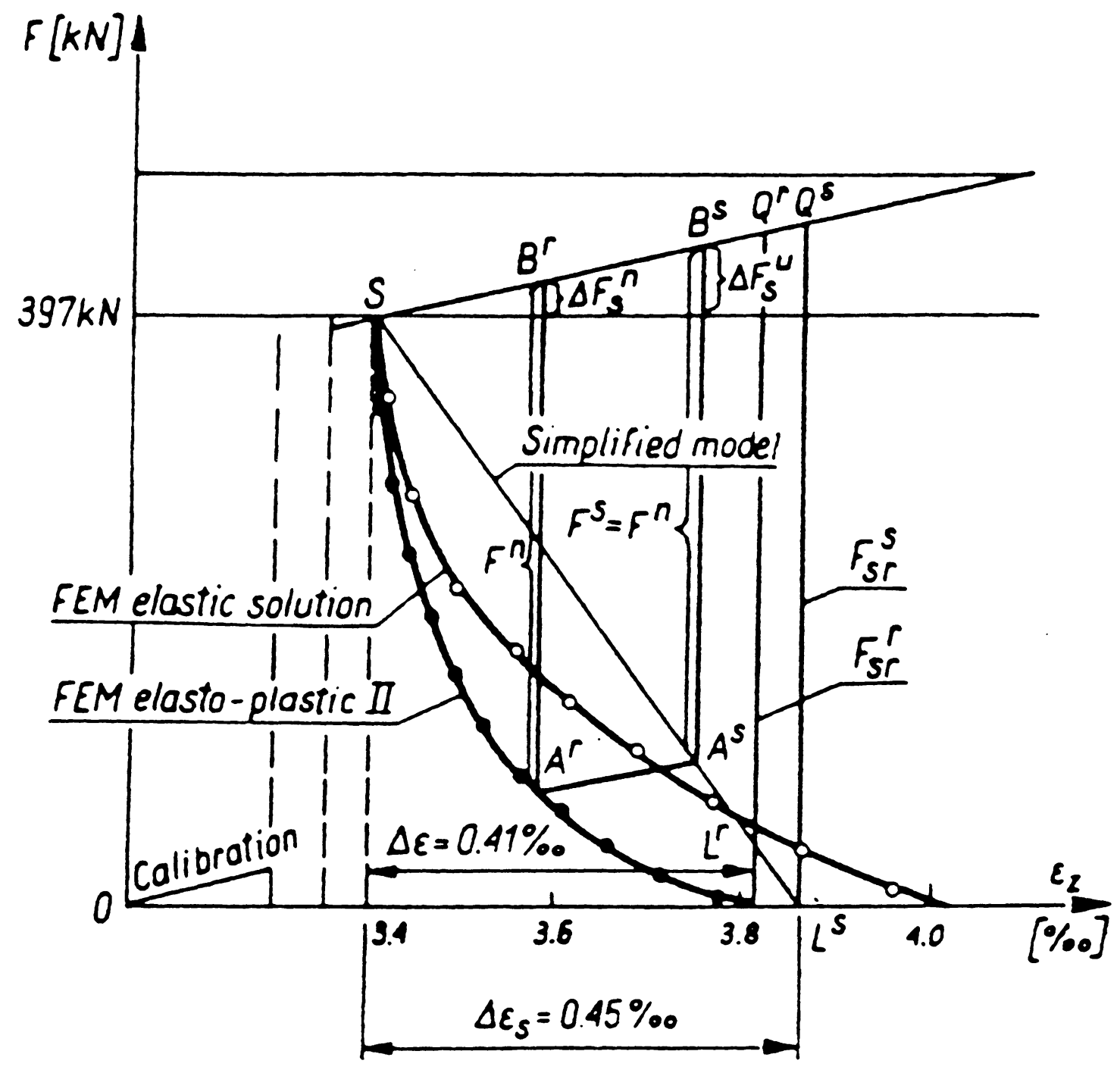

Figure 8. Nonlinear Bolt-Loading Diagram (after Kwiatkowski et al. [27]) 
and stress distribution in the joint in the preload state only. The influence of external axial loads on the joint stiffness has not been studied properly. Kwiatkowski et al. [27] did study this basic nonlinearity of the bolted joint, but they assumed symmetry about the joint interface plane. Thirdly, results have not been incorporated into a useful design methodology that would lead to the improvement of bolted-joint design. The work presented in the following chapters does not have these shortcomings. 


\section{Chapter 3}

\section{Finite Element Modeling of the Bolted Joint}

\subsection{General Modeling Considerations}

Bolted joints are always part of a larger structure or machine. To most accurately analyze bolted joints, one should model the entire system. The results of the system analysis are then used as boundary conditions in a finite element subregion analysis of the bolted joint. However, there are several reasons why this approach is not practical. First, the structure is highly application dependent. One cannot hope to model all types of structures containing bolted joints. Second, structures are generally much larger than the bolted joints they contain. Even if the shape, physical properties, boundary conditions, and loads imposed on the structure were known, computational analysis of the entire system, including a detailed analysis of every bolted joint, is generally not feasible. Finally, while there has been much work on the development of efficient computational tools for the analysis of major components of structures or machines, such as beams, 
plates, shells, and solids, there has been relatively little effort put forth in the analysis of bolted joints. This is particularly unfortunate because the failure of structures and machines often occurs in the joints. Hence, the objective is to accurately analyze the bolted joint without modeling the structure. Of course, all bolted-joint configurations cannot be analyzed, just as one cannot hope to model all structures containing bolted joints. One can, however, infer from the nondimensional results of a particular joint configuration a much greater understanding of bolted joints in general.

To analyze a particular bolted-joint configuration, we need to conceptually remove it from the structure. Therefore, one must define a bolted-joint region which must be large enough to contain the preload interface contact area and the compressed joint members. At the same time, the region must be small enough not to include any significant portion of the structure.

Once one has defined a bolted-joint region, the region is removed from the structure by applying external forces along the cut boundaries that correspond to the internal forces that exist in the structure at the cut-boundary location. In essence, the free-bodydiagram principle is invoked for the bolted-joint region. Hence, this approach has a physical basis that is applicable to a wide variety of applications. Note that this approach is a significant departure from the majority of the previous work reviewed in Chapter 2. The authors in these works assumed hypothetical external-loading locations, such as underneath the bolt head or as shown in Figure 3 on page 14. While there may be some applications in which external loads are applied directly underneath the bolt head and nut, no such application exists for the loading case of Figure 3 on page 14 . Such a hypothetical loading plane is a convenient contrivance that allows an analytical solution to be obtained based on the one-dimensional spring analogy and linear theory. 
It has no direct physical basis. Usually loads, other than the preload, enter bolted-joint regions as internal forces carried by the joint members. If one considers the joint members as essentially plates, then these internal forces are of the following type: shear loads, moment loads, and in-plane loads. Since the axial joint stiffness is our primary interest, in-plane loads will not be considered here.

In almost all engineering problems, a major challenge is to simplify the problem without significantly degrading solution accuracy. In finite element analysis, the first step is to decide on the necessary dimensional size of the finite element model. As mentioned in Chapter 1 , the bolted-joint region is modeled as a solid of revolution by using a twodimensional axisymmetric analysis. The centerline axis of the bolt is the axis of revolution. This axisymmetric assumption permits analysis of three-dimensional physical problems using two-dimensional mathematical analysis. The axisymmetric assumption introduces only minor geometric approximations, such as a cornerless bolt head and nut, but restricts loading conditions to be axisymmetric.

With the dimension of the analysis chosen, one seeks to further simplify the analysis by selecting which features of the bolted-joint region should be mathematically modeled and which features should not be. The components of the joint which are considered important are the bolt, nut, and both joint members contained in the bolted-joint region. Figure 9 shows the bolted-joint configuration studied in this work. A typical axisymmetric finite element model of this configuration is shown in Figure 10. The finite element model is kept as simple as possible by not modeling washers under the bolt head or nut.

Figure 10 shows that symmetry has not been imposed at the joint interface. The entire bolted-joint region has been modeled. Symmetry was not assumed because the joint is, 


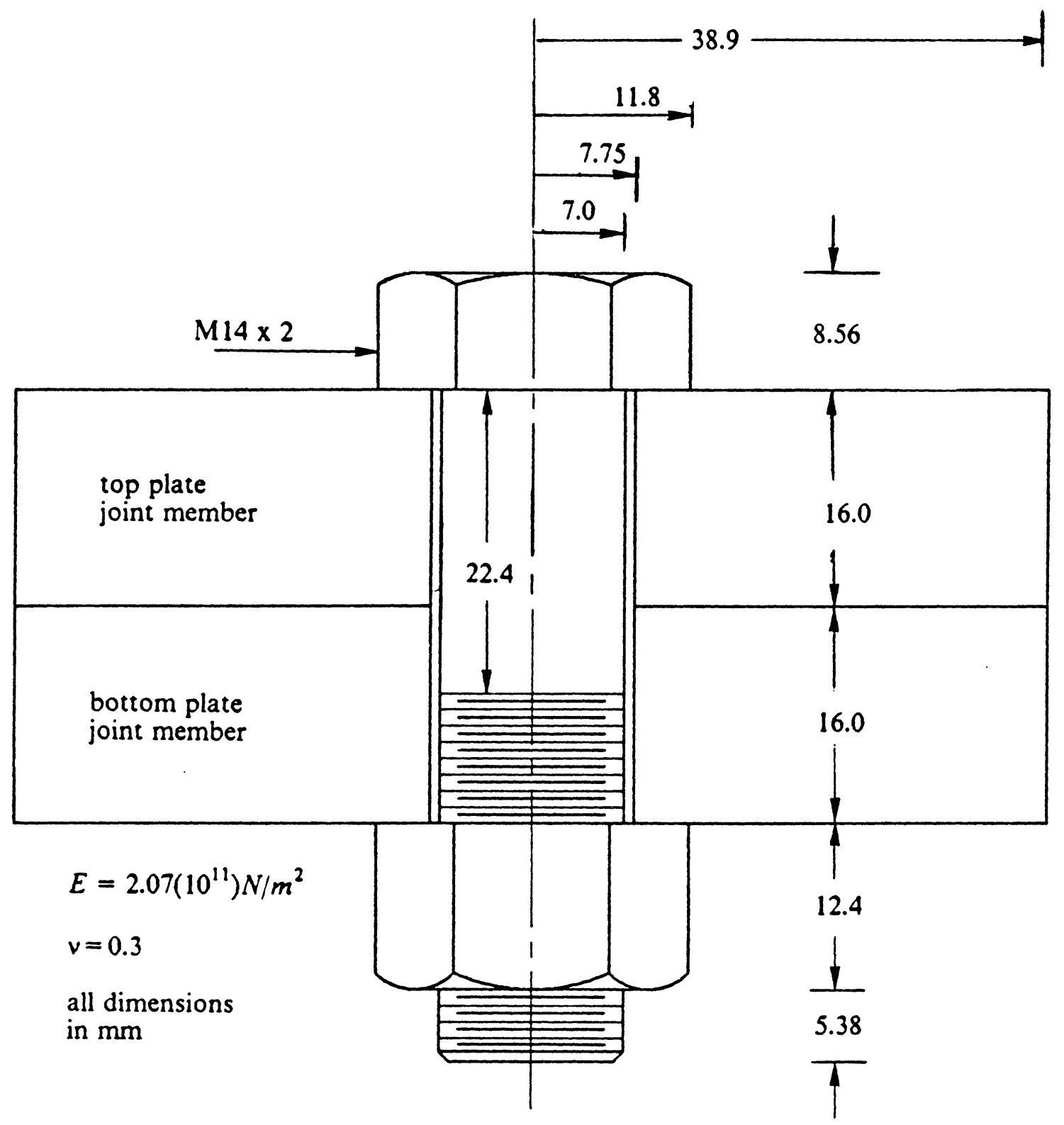

Figure 9. The Bolted-Joint Region Analyzed 


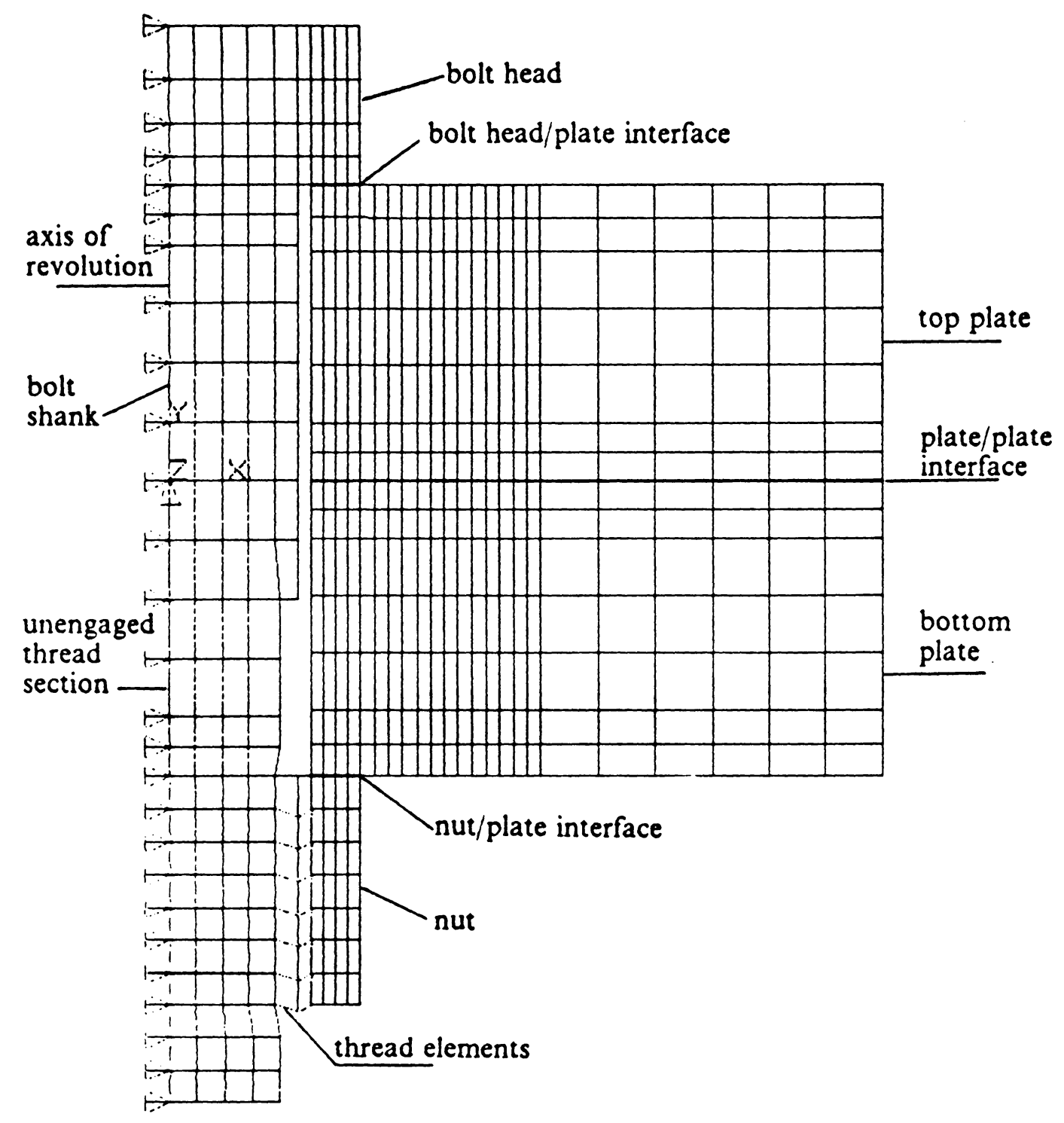

Figure 10. An Axisymmetric Finite Element Model of $A$ Bolted Joint Region 
in fact, not symmetric about the joint interface. For example, based on ANSI Standards ([28], [29]), a metric M14 hex nut, style 1, can have a thickness up to 1.5 times the thickness of the bolt head of a metric M14 hex bolt. Moreover, the nut is only loosely connected to the bolt via the bolt/nut threading, while the bolt head is an integral part of the bolt. One should not, therefore, expect the deformations of the bolt head and nut to be symmetric with respect to the joint interface plane.

Every attempt has been made to ensure that the bolted-joint model conforms to ANSI standards. The bolted joint modeled in Figure 10 on page 29 consists of a M14 x 2 metric hex bolt and nut, and two $16 \mathrm{~mm}$ thick plates with a normal clearance hole diameter of $15.5 \mathrm{~mm}$. The $32 \mathrm{~mm}$ joint thickness is based on ANSI Pipe Flange Standard B16.5 for Class PN20 Flange used with $32 \mathrm{~mm}$ nominal pipe size and M14 bolts. The outer diameter of the nut and the diameter of the bolt head are based on a $20.7 \mathrm{~mm}$ width across the flats found in ANSI Standards B18.2.4.1M-1979 and B18.2.3.5M-1979, respectively. Also found in these standards are the $8.56 \mathrm{~mm}$ bolt head height and 12.4 mm nut thickness shown in Figure 10 on page 29.

The bolt detail consists of the bolt head, shank, the unthreaded section of the bolt, the threaded engaged section, and the bolt stud. The diameter of the unthreaded bolt section and the bolt stub is calculated from the tensile stress area of the threads. The shank diameter is simply the nominal bolt diameter. Since an accurate stress analysis is not the objective, neither the bolt head/shank fillet or the actual thread profile geometry of the bolt or nut is modeled. However, the effect of the bolt/nut thread interaction has been modeled with an appropriate implementation of Bretl's [25] special orthotropic "thread" elements. These elements and their properties are discussed later in this chapter. 


\subsection{Modeling the Joint Interface}

The bolted-joint model of Figure 10 on page 29 has three important interfaces in which sliding or separation may occur: the bolt head/top plate interface, the nut/bottom plate interface, and the top plate/bottom plate interface. These interfaces are modeled using the two-dimensional, axisymmetric gap elements (STIFF12 elements in ANSYS). The two-noded element connects nodes across the interface or gap with a stiffness function that depends on the forces imposed on the element. A high stiffness is activated when a normal compressive force occurs in the element, corresponding to the situation of interface contact at that nodal location. The high stiffness allows transmission of the compressive force across the interface. A zero stiffness is associated with the element when a positive normal force occurs in the element, i.e. the interface separates. The positive normal force is not transmitted across the interface. As the mesh is refined in the interface region, more gap elements are used to join nodes across the interface and the interfacial pressure distribution becomes more accurate.

If the normal force is compressive, the element also has the ability to model Coulomb friction along the joint interface. The element acts as a linear spring in tangential direction with the magnitude of the tangential or friction force limited by the coefficient of friction $\mu$ times the magnitude of the compressive normal force $F_{n}$. The compressive normal stiffness is used as the tangential stiffness. If the magnitude of the tangential force, $F_{s}$, exceeds $\mu\left|F_{n}\right|$, sliding occurs and the element has zero stiffness in the tangential direction. Figure 11 shows the force-deflection relationships for this element. This element is the source of the nonlinearity in the finite element model. If any of the interface elements changes stiffness in either the normal or tangential direction, a new 

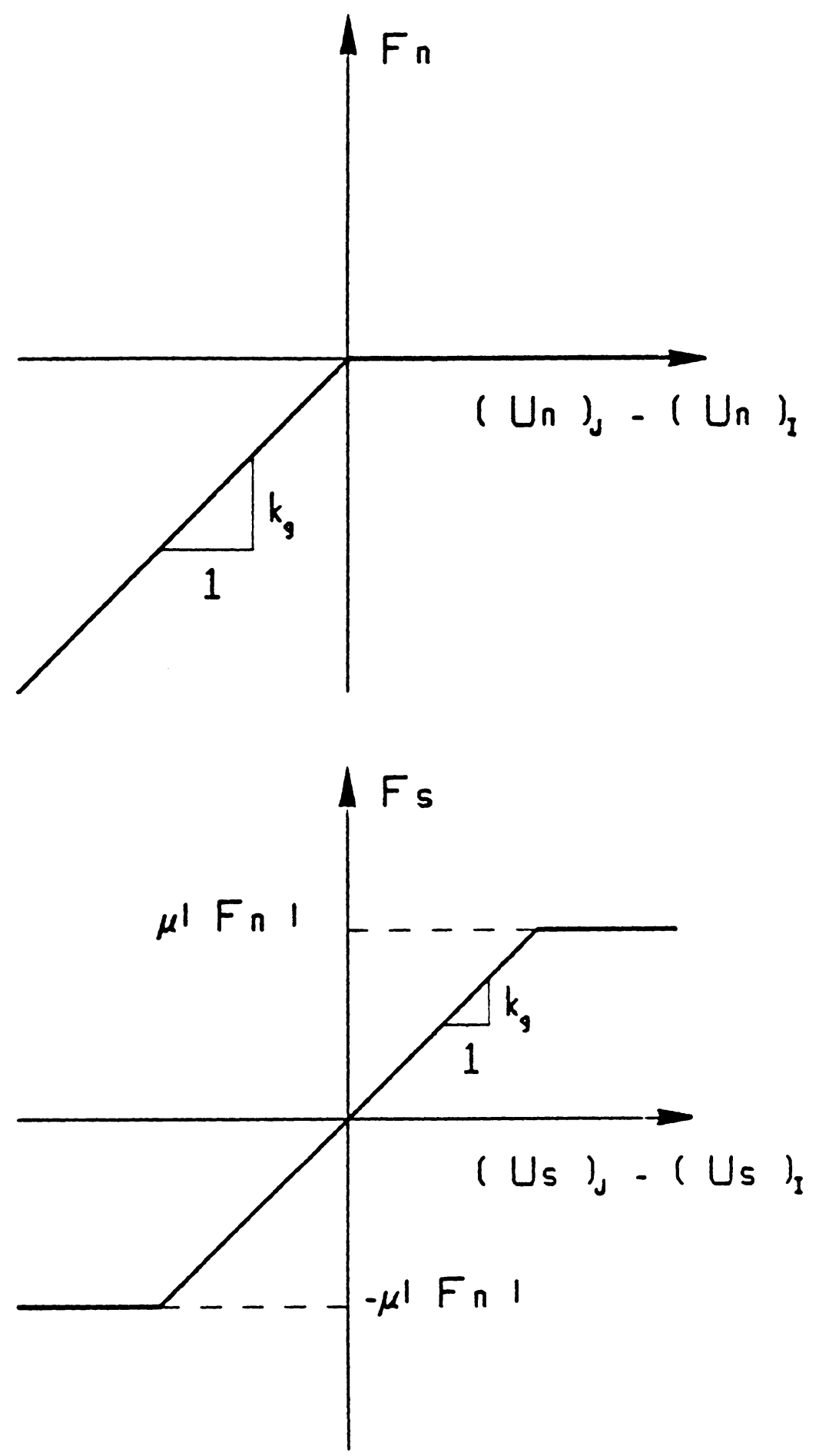

Figure 11. Gap Element Load-Deflection Relationships 
iteration is needed to reformulate the structural stiffness matrix, solve the system of equations, and calculate stresses. Convergence is not achieved in ANSYS unless there has been no changes in any of the gap element stiffnesses in two successive iterations.

The gap element requires two constants: the coefficient of friction $\mu$ and the gap element stiffness $k_{g}$. The coefficient of friction can be estimated from engineering knowledge of the problem. However, the gap element stiffness is somewhat artificial. The gap elements are not being used to model the stiffness of the interfacial microscopic asperities. Rather, the purpose of the gap elements are to transmit compressive and tangential loads across the interface without introducing macroscopic deformations between the interfacial nodes. Consequently, it is only necessary to set the gap element stiffnesses significantly high so as not to introduce these macroscopic deformations. This was accomplished by using the following formula to calculate the gap element stiffness $k_{g}^{i}$ for the $i^{\text {th }}$ interfacial node.

$$
k_{g}^{i}=10 \frac{E_{e} A_{i}}{L_{e}}
$$

$A_{l}$ is the area associated with the $i^{\text {th }}$ interfacial node. $E_{e}$ and $L_{e}$ are the elastic modulus and thickness in the normal direction of an adjacent planar element. Roughly speaking, Eq. (3.1) sets the gap element stiffness an order of magnitude larger than the adjacent element stiffness in the normal direction. 


\subsection{Modeling Bending in the Bolted Joint}

Because of the possibility of significant bending in the bolt head, nut, and joint members, higher-order incompatible elements, called QM6 elements (introduced by Wilson et al. [30] in 1973), are used instead of the standard QM4 planar element. Figure 12 illustrates the difference between the two elements. The shape functions of the QM6 element include two extra terms which allow the element to take on parabolic deformations along its edges. The coefficients, $a_{i}, i=1,2,3,4$, are internal element degrees of freedom. Four additional equations are generated by minimizing the strain energy of the element with respect to each $a_{i}$. These equations are then used to eliminate these internal degrees of freedom in terms of the nodal degrees of freedom, thereby reducing the $12 \times 12$ element stiffness matrix to the standard $8 \times 8$ element stiffness matrix size of a four-noded planar element. If the element is square, it can model a state of pure bending exactly. In contrast, the QM4 element is too stiff under bending due to a parasitic shear effect (see Ref. [2], pg 190). The QM6 element is incompatible; element edges may overlap or separate. Yet, despite the incompatibilities, the element satisfies convergence criterion if it has a parallelogram shape.

QM6 elements are used for all planar elements outside of the bolt shank radius. QM4 elements are used for elements inside the bolt shank radius for two reason. First, bending is not significant in this region. Second, Cook [2, pp 228-229] notes that the axisymmetric QM6 element can create spurious shear strains for elements close to the axis of revolution. Since it was not known whether ANSYS accounts for this potential problem, QM4 elements were used in this region to avoid possible numerical error. 

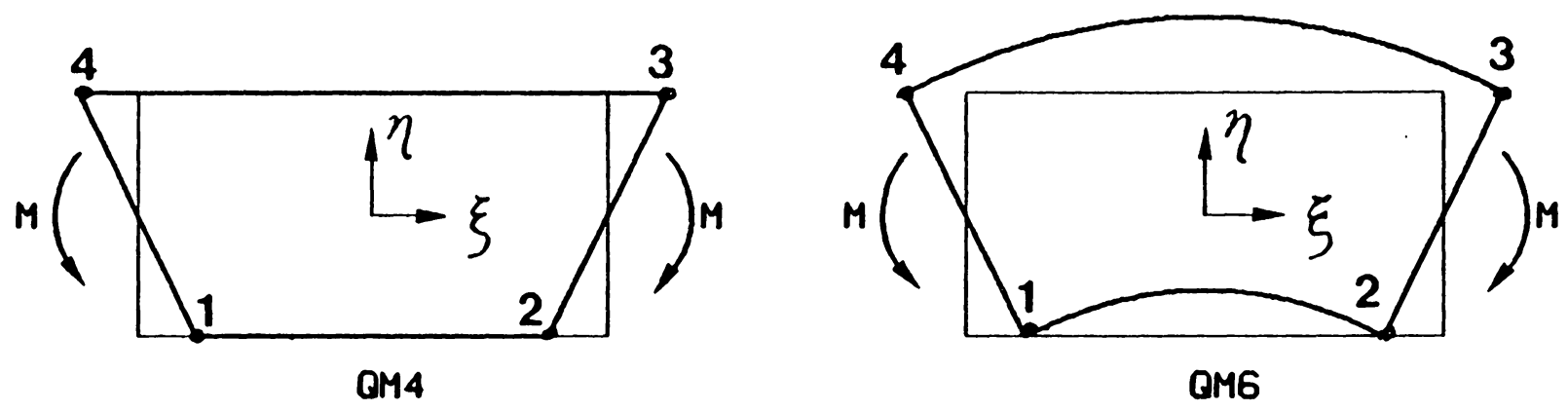

$$
\begin{aligned}
& u=\sum_{i=1}^{4} N_{i} u_{i} \\
& v=\sum_{i=1}^{4} N_{i} v_{i}
\end{aligned}
$$$$
u=\sum_{i=1}^{4} N_{i} u_{i}+\left(1-\xi^{2}\right) a_{1}+\left(1-\eta^{2}\right) a_{2}
$$$$
v=\sum_{i=1}^{4} N_{i} v_{i}+\left(1-\xi^{2}\right) a_{3}+\left(1-\eta^{2}\right) a_{3}
$$$$
N_{i}=\frac{1}{4}\left(1-\xi_{i} \xi\right)\left(1-\eta_{i} \eta\right)
$$$$
\left\{\eta_{i}\right\}=\left[\begin{array}{llll}
-1 & 1 & 1 & -1
\end{array}\right]^{T}
$$$$
\left\{\xi_{i}\right\}=\left[\begin{array}{llll}
-1 & -1 & 1 & 1
\end{array}\right]^{T}
$$$$
i=1,2,3,4-\text { nodal index }
$$ 


\subsection{Modeling the Effect of the Bolt/Nut Thread}

\section{Interaction}

The effect of the bolt/nut thread interaction was modeled without modeling the actual bolt and nut thread geometry by using special orthotropic elements developed by Bretl [25]. The author developed a special orthotropic material stiffness matrix for planar axisymmetric QM4 elements in the bolt/nut thread region which simulates the effect of the bolt/nut threading interaction. The main interaction effect which Bretl simulated with these elements was the radial expansion of the nut. This radial expansion is significant because the nut is only loosely attached to the bolt via friction in the bolt/nut threading.

Consider the load on a single thread as shown in Figure 13. Bretl first assumed that the $l$ and $m$ directions of Figure 13 are principal stress directions. The general axisymmetric orthotropic material stiffness matrix for the $\ln \theta$ coordinate system is given by

$$
\left[E_{l m \theta}=\frac{1}{D}\left[\begin{array}{cccc}
E_{l l}\left(1-v_{m \theta} v_{\theta m}\right) & E_{m m}\left(v_{l m}+v_{\theta m} v_{l \theta}\right) & E_{\theta \theta}\left(v_{l \theta}+v_{l m} v_{m \theta}\right) & 0 \\
E_{l l}\left(v_{m l}+v_{\theta l} v_{m \theta}\right) & E_{m m}\left(1-v_{l \theta} v_{\theta l}\right) & E_{\theta \theta}\left(v_{m \theta}+v_{m l} v_{l \theta}\right) & 0 \\
E_{l l}\left(v_{\theta l}+v_{m l} v_{\theta m}\right) & E_{m m}\left(v_{\theta m}+v_{l m} v_{\theta l}\right) & E_{\theta \theta}\left(1-v_{l m} v_{m l}\right) & 0 \\
0 & 0 & 0 & G_{l m}
\end{array}\right]\right.
$$

where $D=1-v_{l m} v_{m l}-v_{m \theta} v_{\theta m}-v_{\theta \theta} v_{\theta l}-2 v_{m l} v_{\theta m} v_{\theta \theta}$. Since $l$ and $m$ are taken as the principal stress directions, the shear stress $\tau_{l m}$ must be zero. Therefore, $G_{l m}$ is set to zero in the matrix $[E]_{l m \theta}$. To let the nut expand radially almost independent of the bolt, Bretl released most of the constraint between the nut and bolt by requiring the stress $\sigma_{l}$ to be 


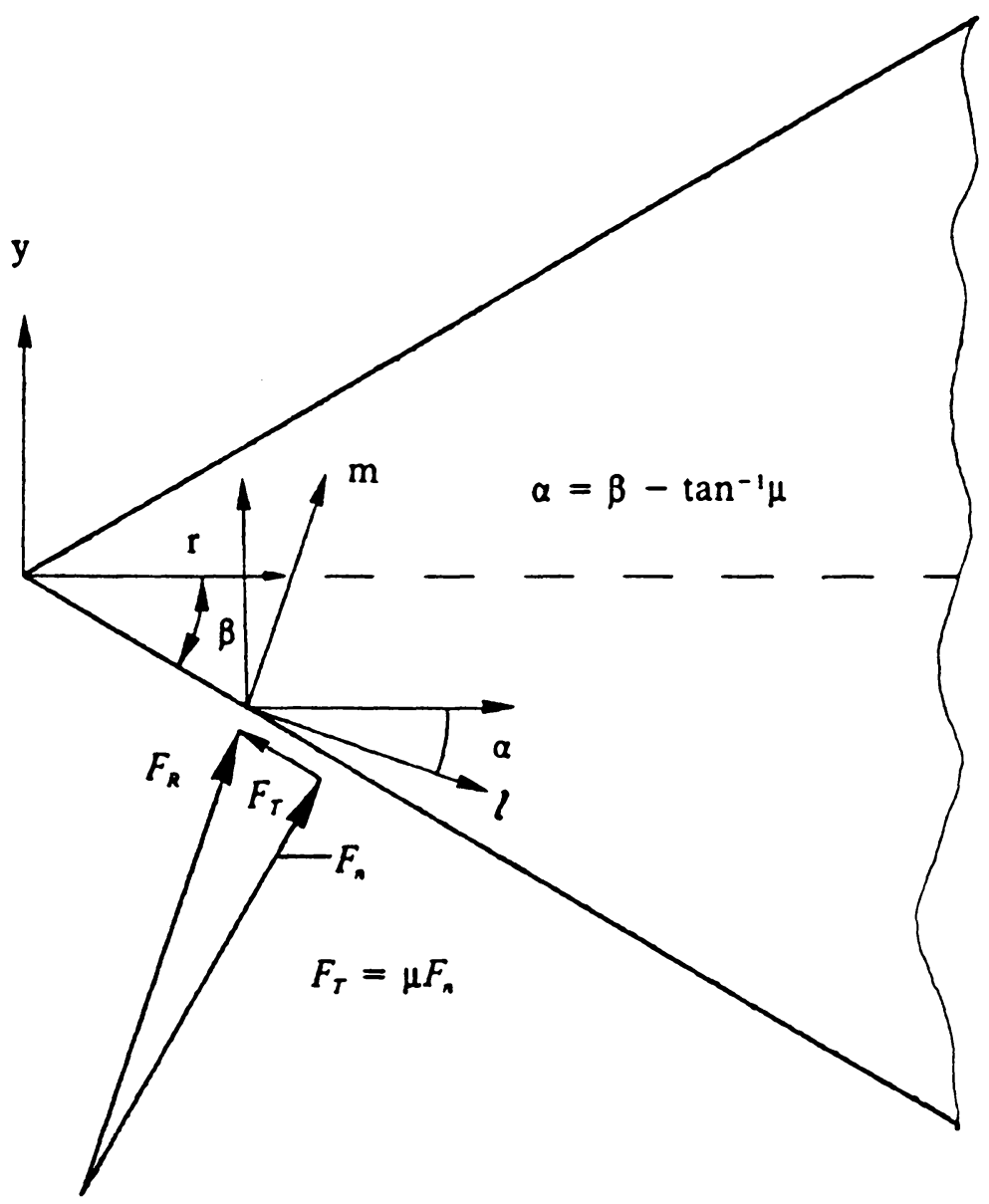

Figure 13. Load on a Thread 
small. Since the other stress components may be large, Bretl set the coupling terms $v_{l m}$ and $v_{\theta \theta}$ to zero, as well as $v_{m l}$ and $v_{\theta l}$ to ensure symmetry. Bretl then allowed normal isotropic conditions to exist in the $y \theta$ plane. Therefore, one has

$$
\begin{aligned}
E_{m m} & =E_{\theta \theta}=E \\
v_{m \theta} & =v_{\theta m}=v
\end{aligned}
$$

The orthotropic matrix of Eq. (3.2) reduces to

$$
[E]_{l m \theta}=\frac{1}{1-v^{2}}\left[\begin{array}{cccc}
E_{l l} & 0 & 0 & 0 \\
0 & E & v E & 0 \\
0 & v E & E & 0 \\
0 & 0 & 0 & 0
\end{array}\right]
$$

The orthotropic matrix $[E]_{l m \theta}$ of Eq. (3.4) is transformed to the $r y \theta$ coordinate system using the fourth-order tensor transformation

$$
[E]_{r y \theta}=[T]^{T}[E]_{l m \theta}[T]
$$

where

$$
[T]=\left[\begin{array}{cccc}
c^{2} & s^{2} & 0 & c s \\
s^{2} & c^{2} & 0 & -c s \\
0 & 0 & 1 & 0 \\
-2 c s & 2 c s & 0 & c^{2}-s^{2}
\end{array}\right]
$$

with $c=\cos \alpha$ and $s=\sin \alpha$. If this coordinate transformation is carried out, matrix $[E]_{r y \theta}$ is found to have shear coupling terms which give the matrix anisotropic characteristics. 
Bretl used the above material properties in special "thread" elements to construct a two-dimensional axisymmetric finite element model of a bolted-joint specimen from which Goodier [31] had obtained experimental data. The author found that by setting $E_{l l}$ to zero, the radial expansion of the nut in his finite element model was in excellent agreement with the experimental data of Goodier.

It is not clear how one can justify assuming the $l$ and $m$ directions of Figure 13 on page 37 to be principle stress directions. Brel offers no evidence to support this assumption. The necessity for making this assumption is not clear. It would seem more natural to formulate an orthotropic material stiffness matrix in a coordinate system with axis normal and tangential to the thread profile. The modulus of elasticity in the tangential direction would be much smaller than the normal isotropic modulus and a function of the thread coefficient of friction. The shear modulus in this system could be reduced to reflect the reduce shear strength of the thread region. The material matrix in this system could then be transformed to the $r y \theta$ global system. However, since Bretl did achieve good agreement with Goodier's experimental data, this author chose to use Bretl's special thread element instead of starting anew and formulating a new thread element.

Before these special "thread" elements were implemented here, it was necessary to verify the ability of ANSYS to handle these special elements. Therefore, a finite element model of Goodier's test specimen, similar to the model used by Bretl, was constructed. Bretl's and this author's models are shown in Figures 14 and 15. In both models axisymmetric QM6 elements were used to model the bolt core, nut wall, and supporting base. The models were preloaded to a $40.7 \mathrm{kpsi}(281 \mathrm{MPa})$ bolt stress by applied traction on the bolt shank. 


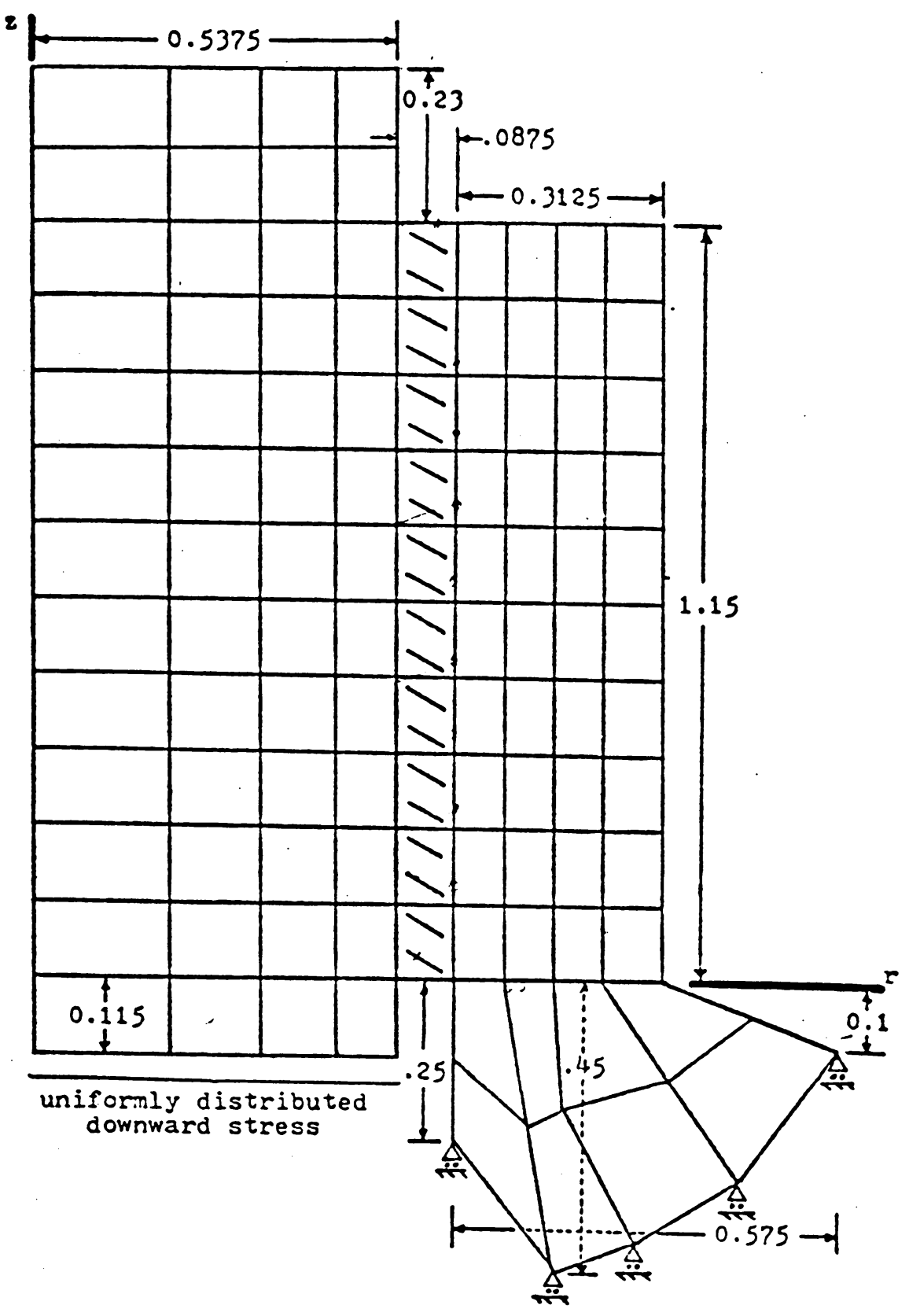

Figure 14. Bretl's FEM of Goodier's Test Specimen (after Bretl [25]) 


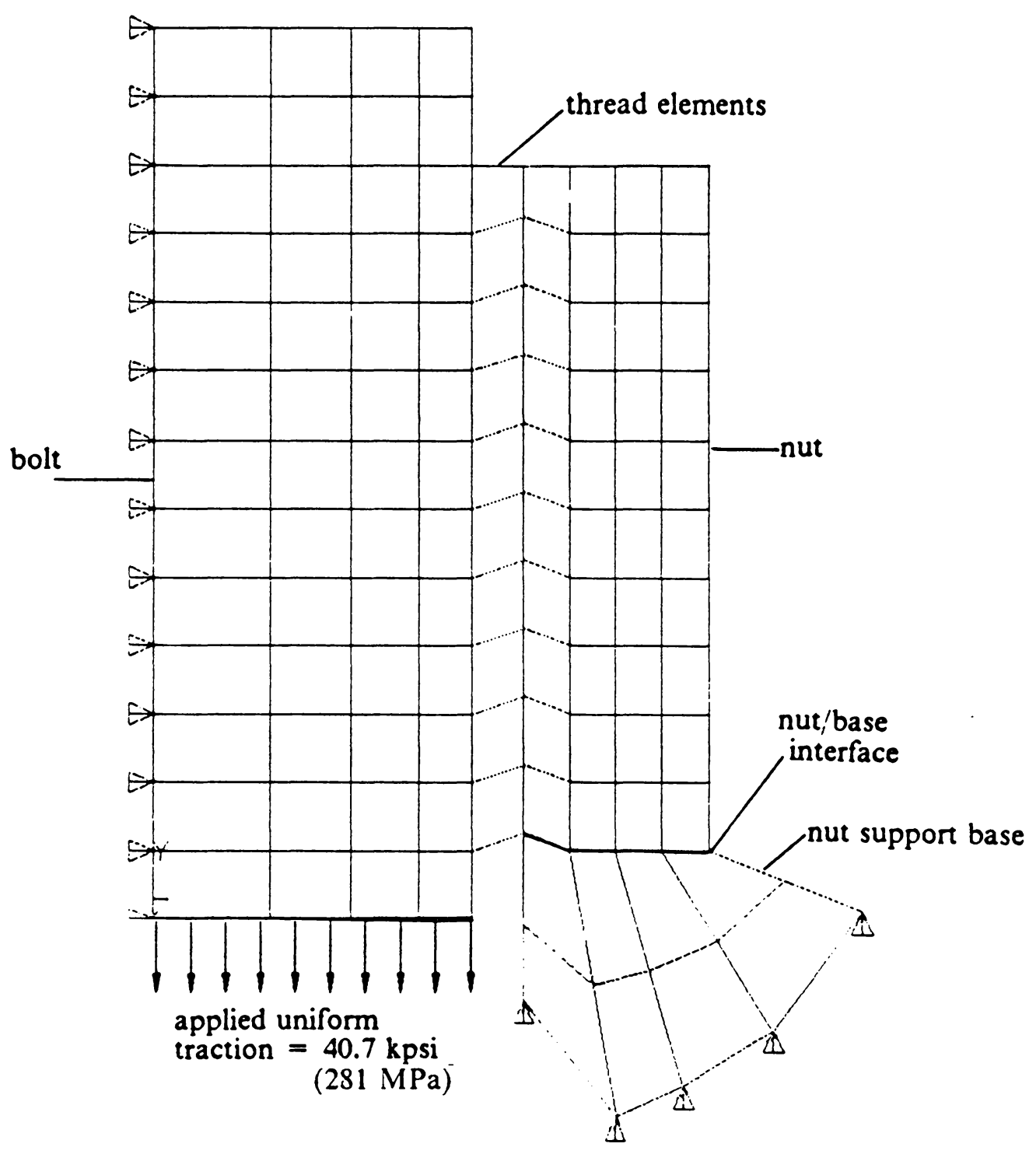

Figure 15. This Author's FEM of Goodier's Test Specimen 
There are several differences between the two models of Figures 14 and 15. To allow sliding with friction at the nut/base interface, Bretl modified the material stiffness matrix of the supporting base elements to allow radial expansion without inducing circumferential stress. He then simulated the friction forces at the nut/base interface by applying loads in the negative radial direction. The total magnitude of these loads was equal to the preload times the coefficient of friction. The model of Figure 15 used conventional material properties for the supporting elements and gap elements to model the nut/base interface.

The other differences between the two finite element models are in the thread element layer. Because ANSYS is limited to orthotropic material properties, the thread elements of Figure 15 cannot have the special thread-element material stiffness matrix $[E]_{r y}$ of Eq. (3.5). The problem was circumvented by inclining the thread elements in Figure 15 on page 41 by the angle $\alpha$ defined in Figure 13 on page 37. The thread elements were inclined $18.7^{\circ}$ based on a $60^{\circ}$ Unified thread angle and a coefficient of friction of 0.2. The matrix $[E]_{l m \theta}$ was then input into ANSYS using the $\ln \theta$ coordinate system of the thread elements. However, Bretl was able to set $E_{l l}=0$ in $[E]_{l m \theta}$. Due to an ANSYS limitation, a zero $E_{l l}$ modulus could not be specified. Instead, $E_{l l}$ was set two orders of magnitude less than $E_{m m}$ and $E_{\theta \theta}$. The transformation of $[E]_{m \theta}$ to $[E]_{r y \theta}$ is carried out within ANSYS.

The preload deformation of the test specimen predicted by a typical ANSYS model using these special "thread" elements is shown in Figure 16. Note the significant amount of radial nut expansion at the nut/base interface. Figure 17 compares the radial expansion of the outside nut surface predicted by various ANSYS finite element models with the experimental data of Goodier and the finite element results of Bretl. Table 1 indicates the differences between the ANSYS finite element models. Excellent matches of 


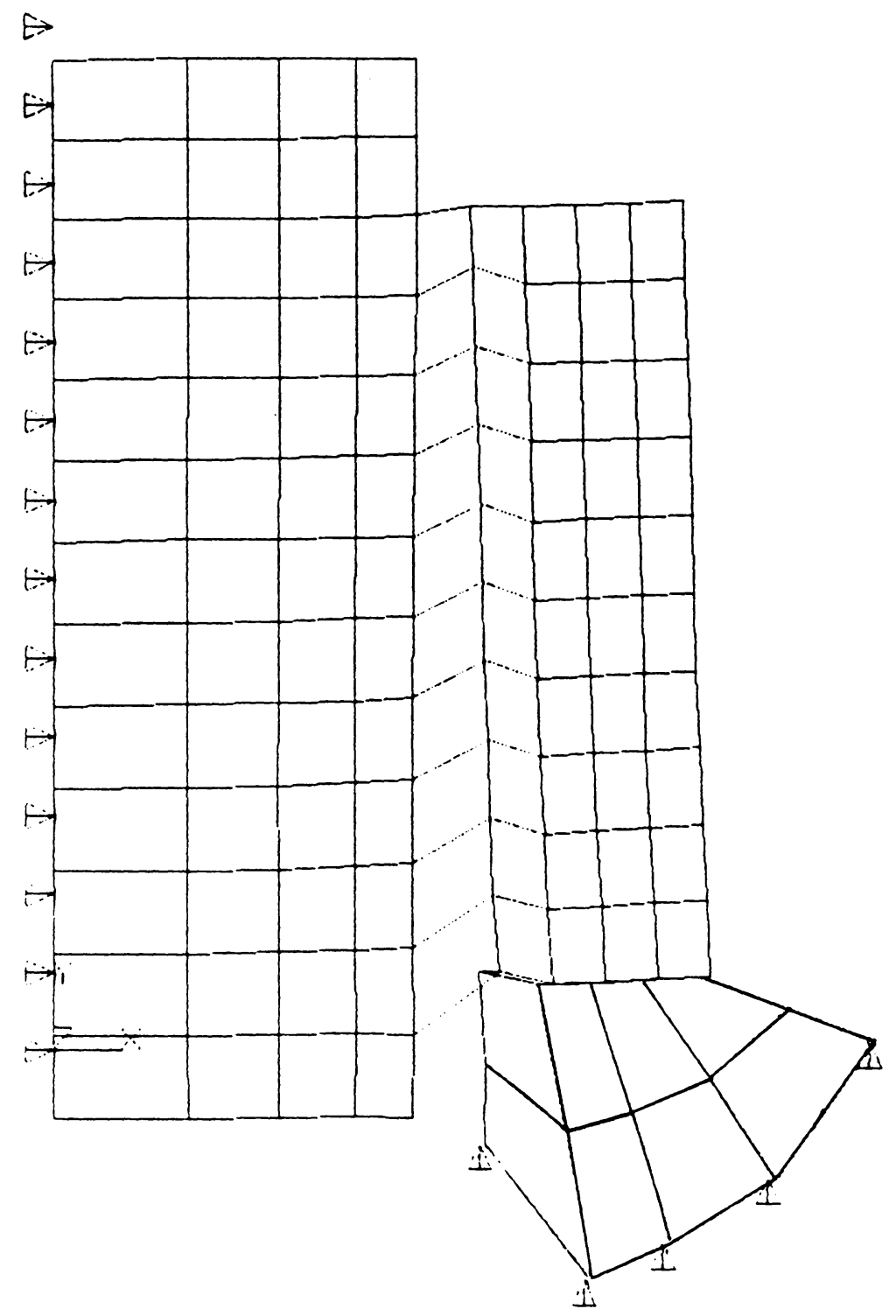

Figure 16. Finite Element Deformation of Goodier's Specimen 


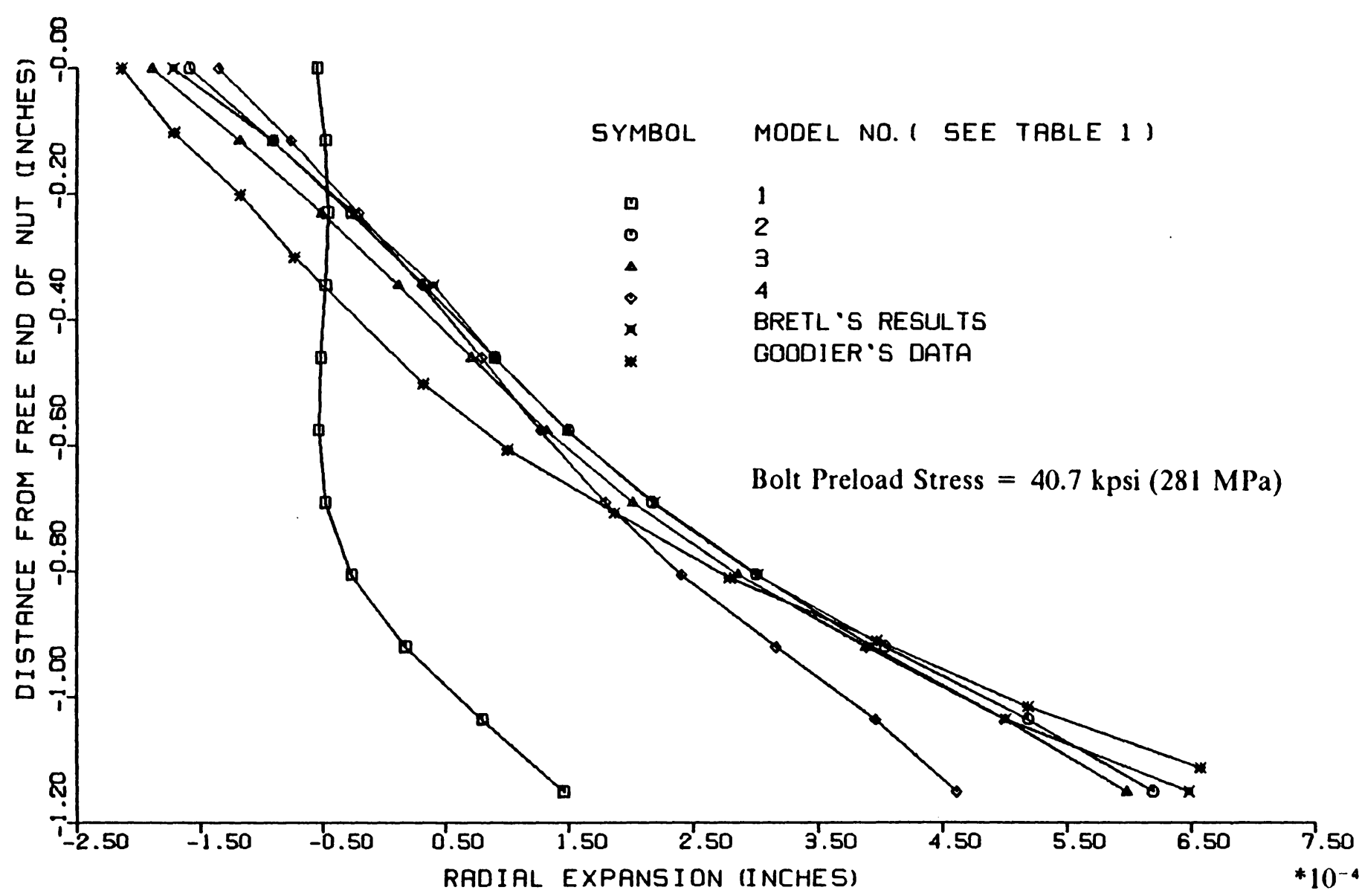

Figure 17. Radial Expansion of the Nut (English units are used to preserve original data of Goodier) 
Table 1. Key to Figure 17.

\begin{tabular}{|c|c|c|}
\hline $\begin{array}{c}\text { Model } \\
\text { No. }\end{array}$ & $\begin{array}{c}\text { Coefficient } \\
\text { of Friction }\end{array}$ & Thread Element Description \\
\hline 1 & 0.1 & normal isotropic QM6 \\
2 & 0.1 & special orthotropic QM4 \\
3 & 0.1 & special orthotropic QM6 \\
special orthotropic QM4 \\
\hline
\end{tabular}

*at the nut/support base interface with simulated

thread-element coefficient of friction $=0.2$ 
Goodier's data are achieved with models 2 and 3. Model 3 offers a slight improvement over model 2. Comparison of the results of model 1 wth the other finite element results and Goodier's experimental data reveals the importance of the orthotropic thread elements in terms of simulating the radial nut expansion. Figure 17 also verifies the implementation of the special thread elements in ANSYS.

\subsection{Preloading the Bolted-Joint Finite Element Model}

The thermal-strain approach was used to preload the bolted-joint finite element model of Figure 10 on page 29. The bolt elements between the bolt head and nut were given a nonzero coefficient of thermal expansion in the axial direction. A uniform temperature below the reference temperature is applied to the model. The resulting axial contraction of the bolt is resisted by the joint members, thereby inducing an axial tensile stress in the bolt. It is important to realize that the bolt preload contraction in the finite element model is not the same as the bolt preload extension in the actual joint under the same preload. Figure 18 illustrates this point. Inducing the bolt preload by cooling the bolt can be viewed as a two-step process. In the first step the bolt undergoes a free axial contraction by the amount $\Delta y_{1}$. No stresses are generated by the free axial contraction. At this point the finite element model simulates the actual bolt without any preload. Next, the bolt preload $F_{l}$ is applied by the reaction forces from the joint members resulting in a bolt preload extension of $\Delta y_{2}$. The result is an observed axial bolt contraction in the finite element model of $\Delta \mathrm{y}=\Delta \mathrm{y}_{1}-\Delta \mathrm{y}_{2}$ for a bolt preload of $F_{i}$. Yet, the bolt axial preload deformation simulated by the finite element model is $\Delta y_{2}$ and is given by 


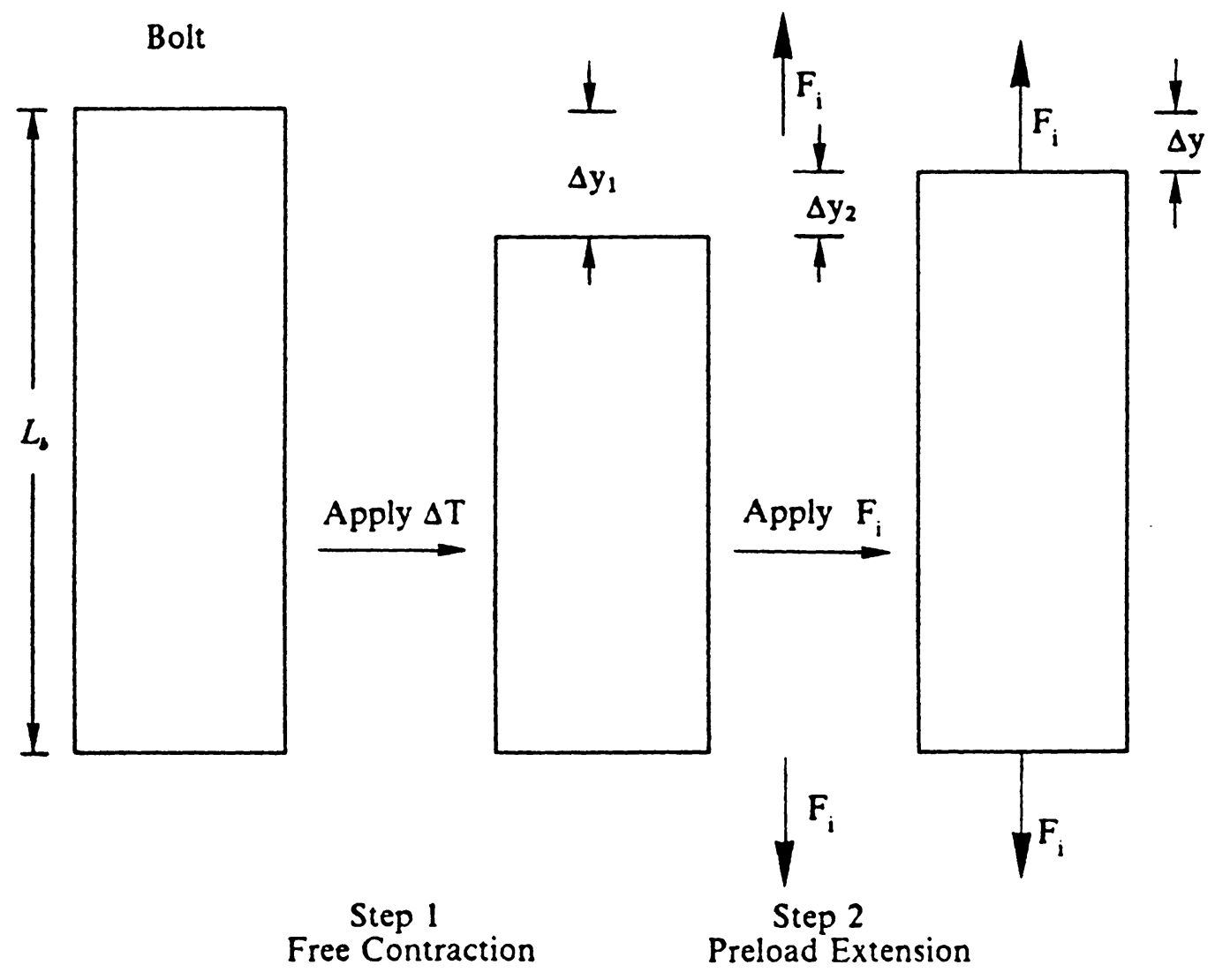

Figure 18. Illustration of Bolt Preload by Thermal-Strain Approach 


$$
\begin{aligned}
\Delta y_{2} & =\Delta y_{1}-\Delta y \\
& =L_{b} \alpha_{y}|\Delta T|-\Delta y
\end{aligned}
$$

where $\alpha_{y}$ is the axial-direction coefficient of thermal expansion.

A high preload in the joint is extremely important in preventing bolt failures. This is due to the fact that increasing the joint preload increases the size of the compressedmember region of the joint. The compressed-member stiffness increases due to the larger area of compression. It can be seen from Eqs. (2.1) and (2.2) that the result is a decrease in the additional load carried by the bolt, $P_{b}$. The recommended design preload depends on the type of loading and the grade of the bolt. For the case of axial static loading, Shigley and Mitchell [7] recommend that the preload be 60 to $90 \%$ of the bolt proof load. The bolt proof load, $F_{p}$, is given by the tensile-stress area $A_{t}$ times the the bolt proof strength $S_{p}$. In some applications preloads of $100 \%$ of the proof load have been recommended [32]. For the case of axial fatigue loading, Shigley and Mitchell recommend a bolt preload of

$$
F_{i}=F_{p}-n C P_{\max }=F_{b}-n P_{b}
$$

where $\mathrm{C}$ is the joint stiffness ratio defined by Eq. (2.2) and $\mathrm{n}$ is the factor of safety. The difficulty in using this equation to calculate the proper bolt preload is obtaining an accurate value for C. C is a function of $k_{m}$, which, in turn, is a function of the external load P.

The bolt preload stress, $\sigma_{i}$, is simply $\frac{F_{i}}{A_{t}}$. For the bolted-joint studied here, a bolt preload stress of approximately $342 \mathrm{Mpa}$ was generated in the finite element model using the thermal-strain approach. This value is $76 \%$ and $90 \%$ of the proof strength of metric 
bolt grades 8.9 and 5.8 , respectively. Achieving the desired preload usually required two converged finite element solutions for a given model to determine the required temperature differential.

\subsection{Loading the Bolted-Joint Finite Element Model}

In accordance with the free-body-diagram approach, loads are applied to the cutboundary of the joint members in the bolted-joint model. The cut-boundary of the joint members is the outer diametric boundary of the bolted-joint region. Figure 19 shows the application of these loads which are considered to be the result of internal forces generated in the joint members. Applied axisymmetric nodal loads are based on two types of axisymmetric internal loads: line shear loads and line radial bending moments. Nodal shear loads are calculated assuming a uniform shear stress $\tau_{x y}$ on the joint edge.

$$
F_{y}^{i}=A^{i} \tau_{x y}=\frac{A^{i} P}{A_{\Gamma}}
$$

where $A^{i}=$ the surface area associated with node $\mathrm{i}, \mathrm{P}=$ total shear load carried at the joint-member edge, $A_{\Gamma}=$ total surface area of the joint-member edge, and $A_{\Gamma}=\Sigma A^{i}$. It should be noted that nodal shear loads calculated using Eq. (3.9) are not "consistent" in a finite element sense. Consistent nodal loads are derived using the same shape functions used to formulate the stiffness matrix. Equation (3.9) is based on the linear, QM4 element shape functions, not the QM6 shape functions of Figure 12 on page 35 used to model bending in the joint. This is because the coefficients, $a_{i}$, in the QM6 shape functions are computed internally in ANSYS and are not known by the user. 


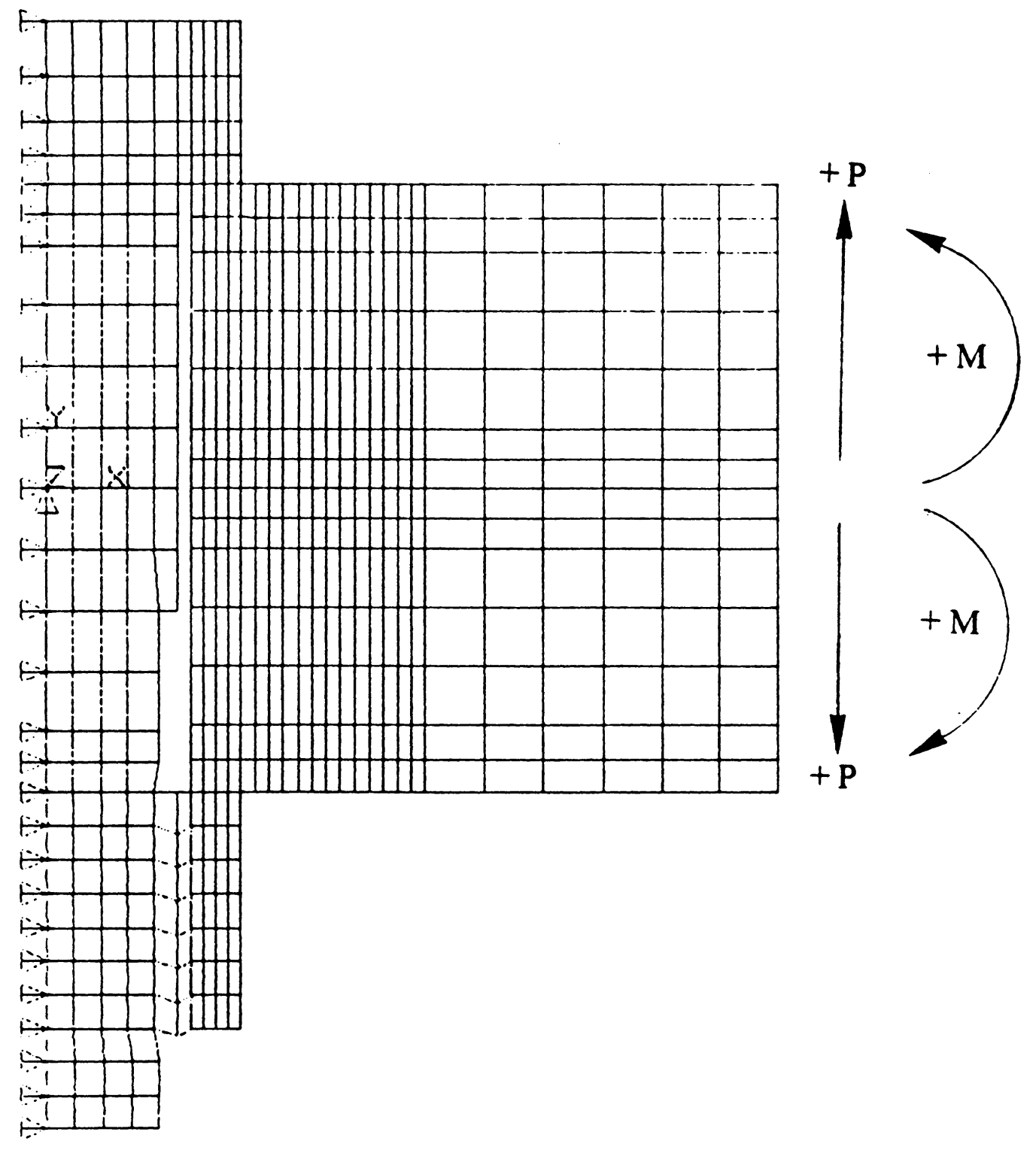

Figure 19. Application of Loads to the Bolted-Joint Model. 
However, the distribution of nodal shear loads at the joint-member edge should not have a significant affect on the axial stiffness of the joint due to Saint Venant's principle.

For a planar axisymmetric finite element model, an applied radial bending moment can be approximated by applying radial nodal forces derived from a pure radial bending stress distribution. Figure 20 shows the pure radial bending stress distribution $\sigma_{r}(y)$ applied to the bolted-joint finite element model. The radial load for node $j$ of element $e$, ${ }^{e} F_{r}^{j}$, is given by

$$
{ }^{e} F_{r}^{j}=2 \pi R_{o} \int N_{j}\left(R_{o}\right) \sigma_{r}(y) d y
$$

where $R_{o}$ is the radius of the joint member edge and $N_{j}\left(R_{o}\right)$ is the shape function for node $\mathrm{j}$ evaluated at $R_{o}$. As in the case of the nodal shear loads, the QM4 shape functions were used in the above computation.

The radial bending stress distribution has the form

$$
\sigma_{r}(\bar{y})=\left(\sigma_{r}\right)_{\max } \bar{y} / L
$$

where $\bar{y}$ is the distance from the neutral axis and $L$ is the joint member thickness. Radial nodal loads along the joint member edge were first calculated in terms of $\left(\sigma_{r}\right)_{\max }$ using Eqs. (3.10) and (3.11). $\left(\sigma_{r}\right)_{\max }$ was then obtained by equating the moment about the neutral axis due to the radial nodal loads to the applied radial moment.

Positive applied shear loads are defined as loads that pull the joint members apart. Positive applied moment loads are defined as loads that bend the plates apart at the joint-region edge. Shear loads were applied to the bolted-joint finite element model in the range from $-1.2 F_{i}$ to $1.2 F_{i}$. For most cases a shear load of 1.2 times the bolt preload 


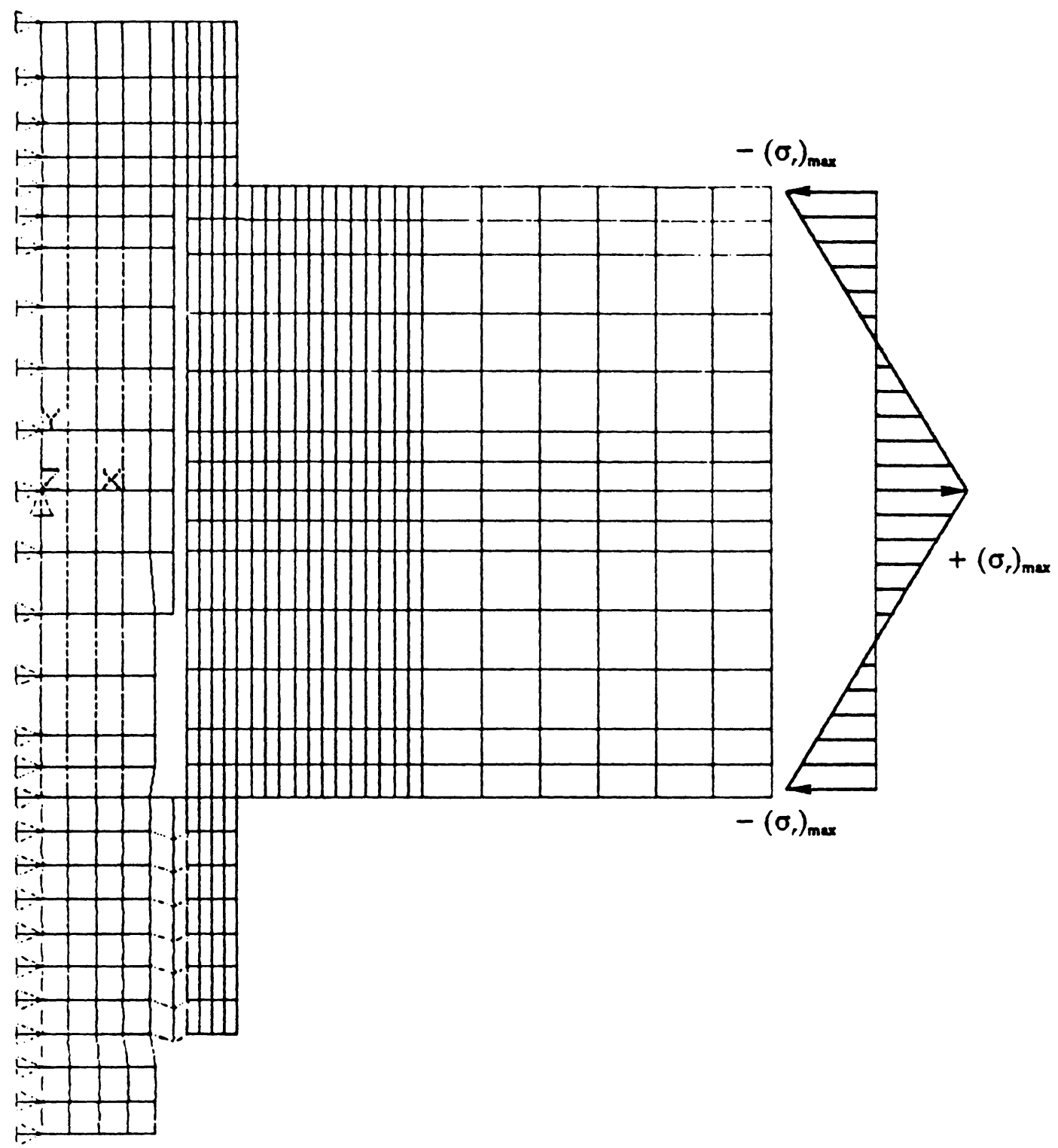

Figure 20. FE.M with Applied Stress Distribution to Simulate Positive Radial Bending Moment 
was sufficient to cause complete joint separation. Complete joint separation is generally regarded as joint failure.

Whereas joint separation provides a reasonable upper bound on the applied axisymmetric shear load, there are no convenient bounds on the applied axisymmetric radial moment load. To estimate a reasonable upper bound on the applied moment, a theoretical plate analysis was carried out. Figure 21 shows the plate geometry studied. The maximum applied tensile shear load, $1.2 F_{i}$, on the bolted-joint model was applied to the plate at the outer radius $a$. The internal radial moment was then calculated at the radius $r=5 b$ which is the outer radius of the bolted-joint model of Figure 10 on page 29. Hence, this internal moment provides an estimate of the maximum moment to apply at the outer edge of the bolted-joint model due to a distant shear load. Both pinned and fixed boundary conditions are considered on the plate inside edge. The actual boundary condition on the inside edge of the bolted-joint member is considered to be bounded by these boundary conditions. The outside edge of the plate is taken as free or guided. The pinned and fixed boundary conditions at the outside edge are of no practical value since the shear load would be taken up completely by reaction forces.

Part b) of Figure 21 shows the radial line moment at $r=5 b$ as a function of the outer plate radius $a$ for the various boundary conditions considered. The radial moment equations from Roark and Young [33] are presented in Appendix A. The radial line moment has been nondimensionalized by the applied shear load/radian. An estimate of the upper bound on the applied moment was obtained by averaging the internal radial moments at $r=5 b$ based on an outer plate radius of $10 b$ for the fixed/free and pinned/free boundary conditions. Plate radii greater than $10 b$ were not considered real- 

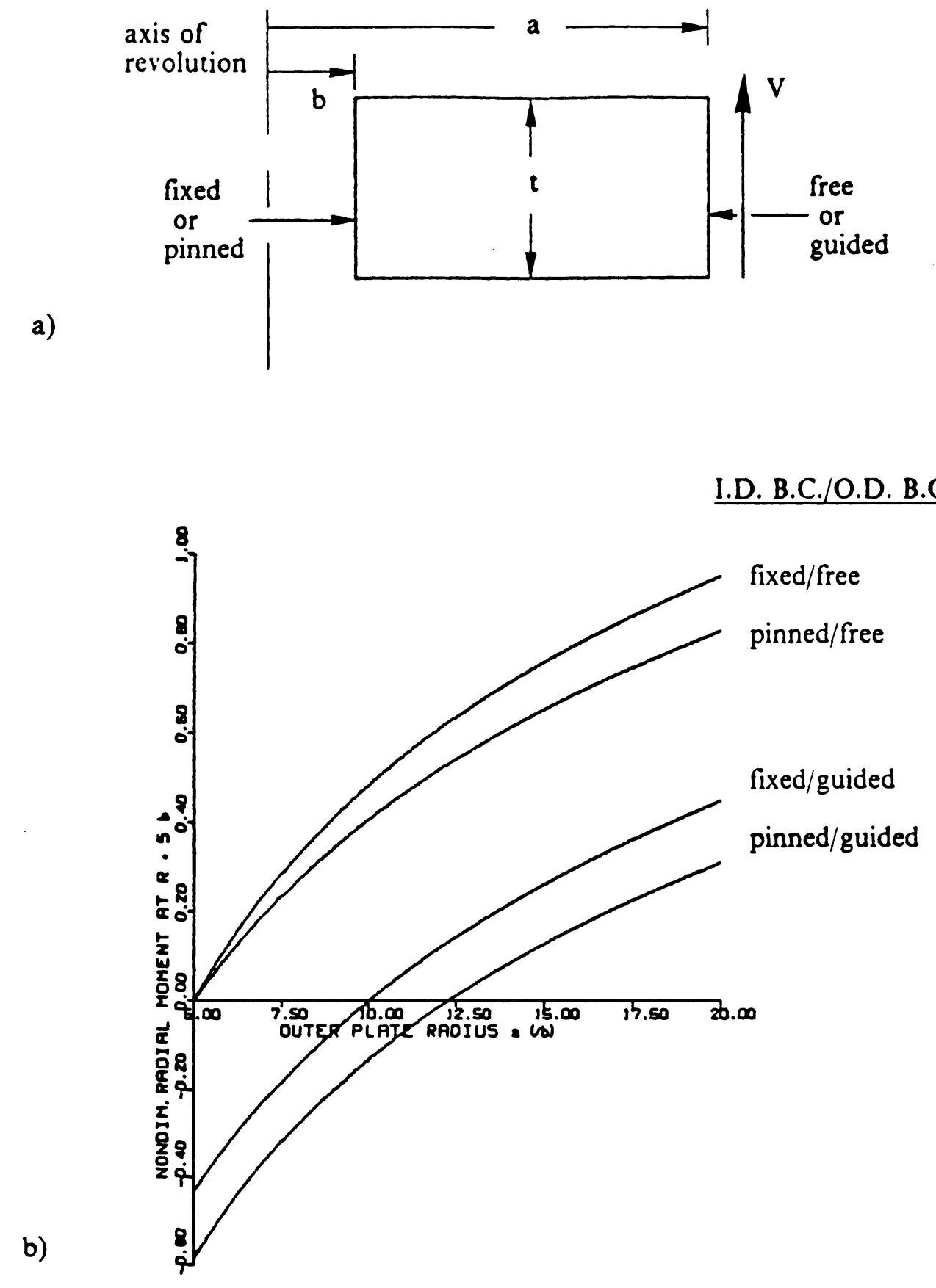

Figure 21. a) Plate Geometry b) Radial Moment at $r=5 b$ as a function of outer plate radius a 
istic because this corresponds to bolt spacings greater than 20 times the nominal bolt diameters for multiple bolt patterns.

Because of the nonlinearity of the bolted-joint model, one cannot superimpose the finite element results due to shear loading only with the finite element results due to moment loading only. Instead, separate results were obtained for the case of shear loading only, moment loading only, and combined moment and shear loading. In the case of the combined moment and shear loading, only positive shear loads and moments were considered. Furthermore, both the shear load and applied moment were incremented together from the preload condition (no loads) to their respective maximum values. This was in accordance with the view that the radial moment entering the bolted-joint region is a result of a distant shear load in the joint member.

\subsection{Final Modeling Notes}

Because nonlinear finite element models must iterate before a converged solution can be obtained, it is important to minimize the solution time (i.e. cost) of each iteration. Since ANSYS employs a frontal solution scheme, the solution time is proportional to the square of the mean wave-front size. The wavefront is the number of active equations in memory at a given instant and is controlled by the element numbering sequence. Hence, the bolted-joint finite element models were wavefront optimized by element number reordering to minimize the model wavefront. 
To prevent rigid-body motion, one node in the finite element model was constrained from motion in the axial direction as seen in Figure 10 on page 29. Nodes on the axis of revolution were also constrained from radial motion. Although theoretically these nodes are incapable of radial motion, numerical error may cause some radial motion. Hence, it is always advisable to radially pin axis-of-revolution nodes in an axisymmetric analysis. 


\section{Chapter 4}

\section{Convergence of the Bolted-Joint Finite Element}

\section{Model}

Because the finite element method is a numerical method which renders an approximate solution, it is important to verify the convergence of the results to a solution. Hopefully, the converged solution and the solution of the problem being modeled are identical. However, due to modeling approximations, assumptions, errors, etc., the converged solution may not be the solution to the actual problem. An important step, though, is to ensure that a converged solution has been obtained.

The solution convergence discussed here should not be confused with equilibrium convergence. For the bolted-joint model, each load step analysis must iterate because the structural stiffness matrix depends on the displacements of the gap element nodes which are not known apriori. If the gap element displacements result in a change in the structural stiffness matrix, equilibrium has not been satisfied and a new iteration is 
started. All finite element results presented in this dissertation converged in an equilibrium sense.

The most obvious reason for nonconverged solutions in a finite element analysis is due to a discretization error- the representation of a continuum or continua with a finite number of degrees of freedom. The discretization error can be reduced by mesh refinement provided that converging elements are used. As mention in Chapter 2 , the incompatible QM6 elements have been shown to satisfy convergence criteria if they have a parallelogram shape, although the convergence is not monotonic as in the case of compatible elements. Mesh refinement is most important in areas of high stress gradients and in geometrically nonlinear regions.

In view of the above discussion, a series of convergence studies was carried out on the bolted-joint finite element model. Convergence was assessed in terms of the axial strains induced in the bolt over multiple shear loads. This definition of convergence was selected because the objective of this work is to characterize the relationship between the applied loads and the bolt load which is proportional to the bolt strain. Mesh refinement was in the radial direction in the bolt head, nut, and joint member regions. This particular mesh refinement scheme was chosen for several reasons. The nonlinearity of the bolted-joint model is due to the gap elements on the model interfaces in these regions. Radial mesh refinement increases the number of gap elements along the interfaces and thereby reduces the interface contact discretization error. In addition, stress gradiants in these regions were significantly larger in the radial direction. Furthermore, calculations based on bolt loads and bolt strains yield an axial bolt stiffness less than $4 \%$ stiffer than the theoretical axial bolt stiffness based on $\frac{E A}{L}$ calculations. Hence, mesh refinement in the bolt region was not considered critical. 
Figures 22 through 24 show the finite element meshes used in the first convergence study. The number of element columns in the compressed joint member region between $\mathrm{r}=10.4 \mathrm{~mm}$ (edge of the bolt head) to $\mathrm{r}=20.2 \mathrm{~mm}$ (end of the preload contact area at the plate/plate interface) were successively increased to double the number of gap elements in this region for each new mesh. Because the gap element stiffness is proportional to the area associated with the interfacial gap-element node by Eq. (3.1), each new mesh scheme required recalculation of the gap element stiffnesses.

Figure 25 is a plot of the applied shear load divided by the bolt preload versus the bolt strain relative to the preload strain. The bolt strain was calculated across the length of the bolt between the bolt head and nut using the axial displacement of the nodes on the bolt centerline. From Eqs. (2.1) through (2.4), it is clear that the slope of the load versus bolt strain curve is the total joint stiffness $k_{T}$ given by Eq. (2.3) and nondimensionalized by the factor $L_{b} / F_{i}$. Figure 25 reveals no significant differences between the load versus bolt strain curves for this convergence study.

The second convergence study investigated the effect of mesh refinement from the edge of the bolt head to the end of the bolted joint region. Six, twelve, and twenty-four element columns were used in this region in the meshes of Figures 26 through 28 . The applied shear load versus bolt strain results are shown in Figure 29. It can be seen that for applied loads from 0 to $80 \%$ of the preload an oscillatory convergence of the load curves occurs. This behavior can be seen more clearly in Figure 30, a zoomed plot of Figure 29. Oscillatory convergence is a result of using the incompatible QM6 elements discussed in Chapter 3. One cannot expect monotonic convergence when using incompatible elements. The solution convergence appears to have been achieved for mesh No. 3 shown in Figure 28. 


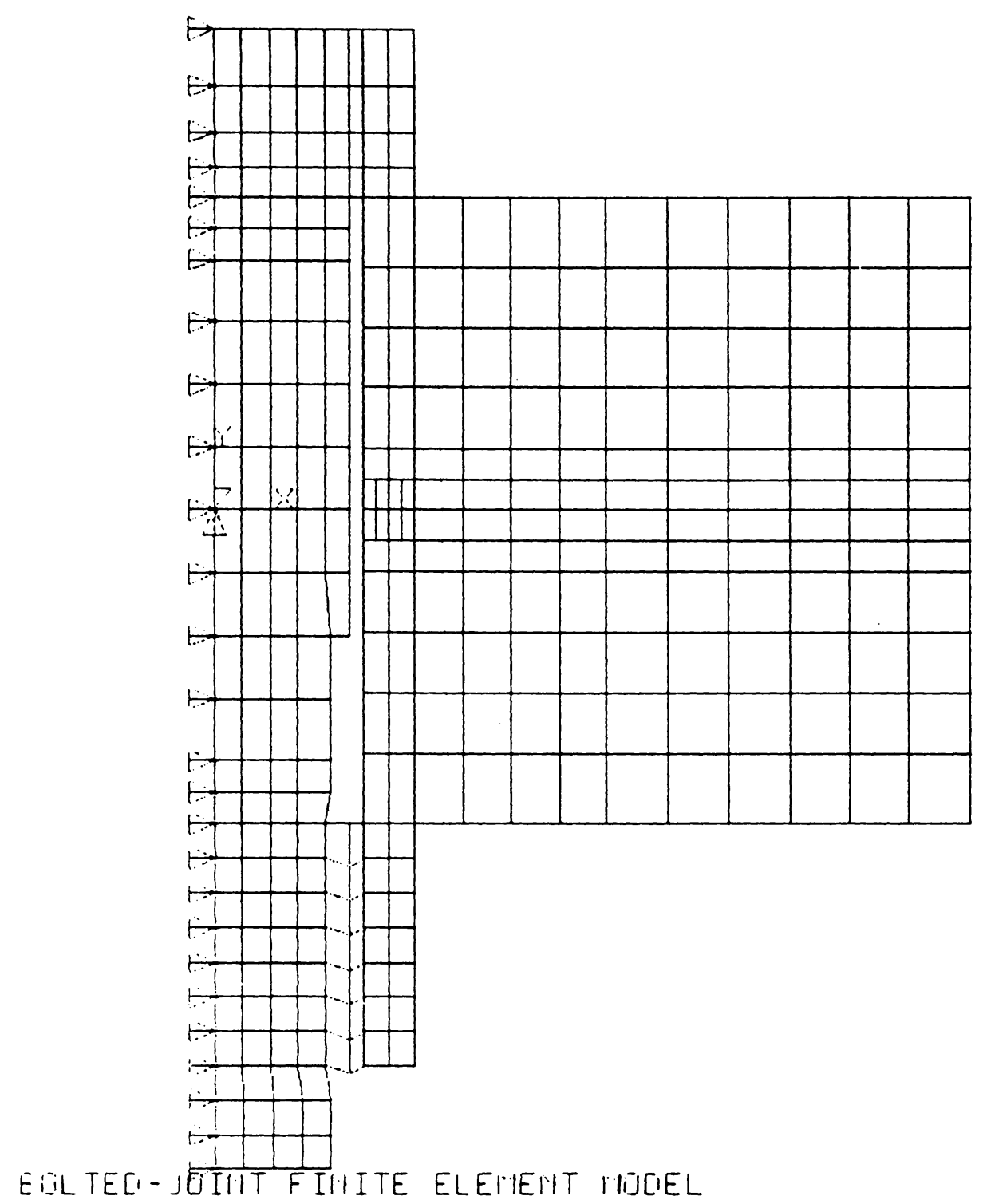

Figure 22. The Bolted-Joint Finite Element Model: Convergence Study No. 1, Mesh No. 1 


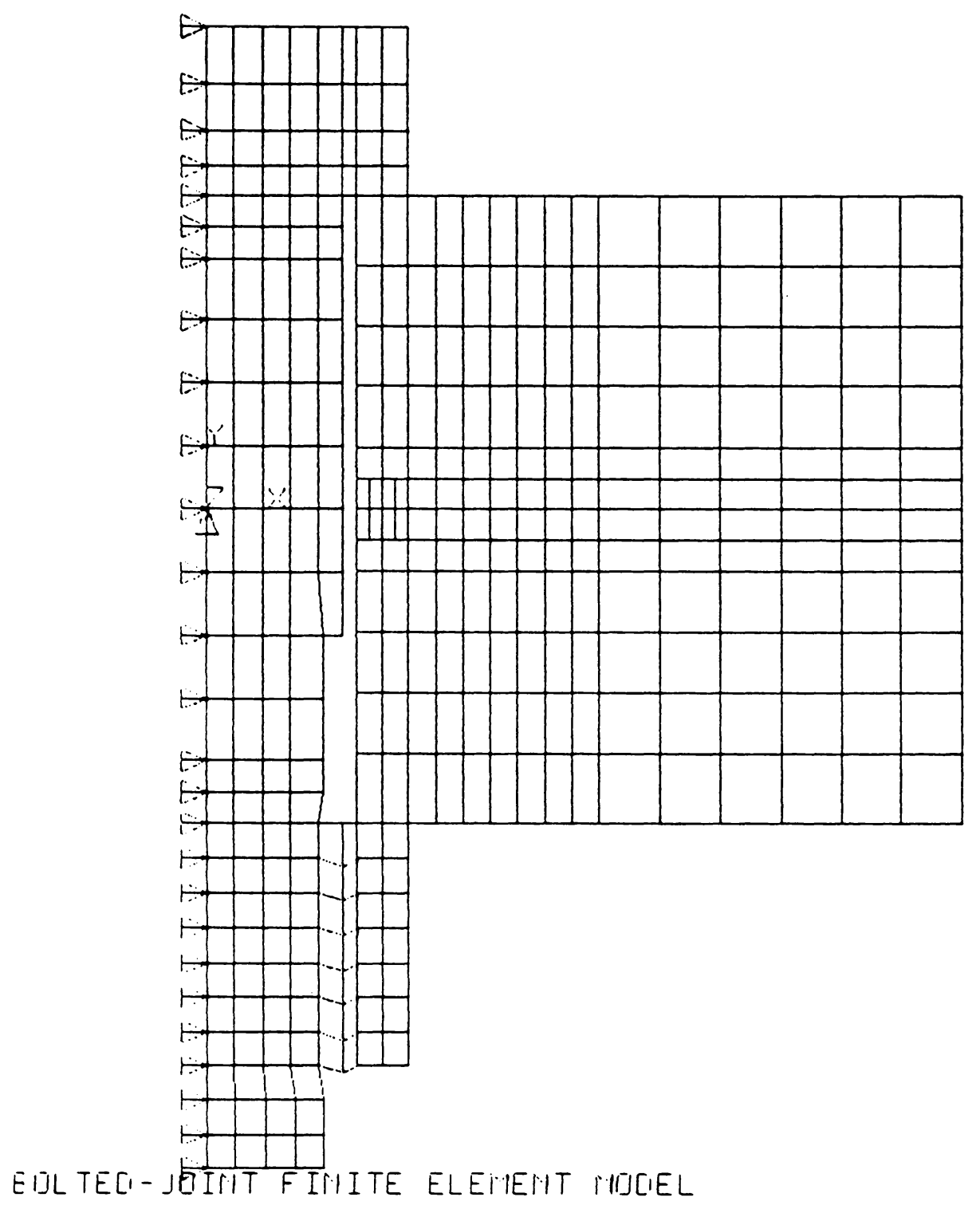

Figure 23. The Bolted-Joint Finite Element Model: Convergence Study No. 1, Mesh No. 2 


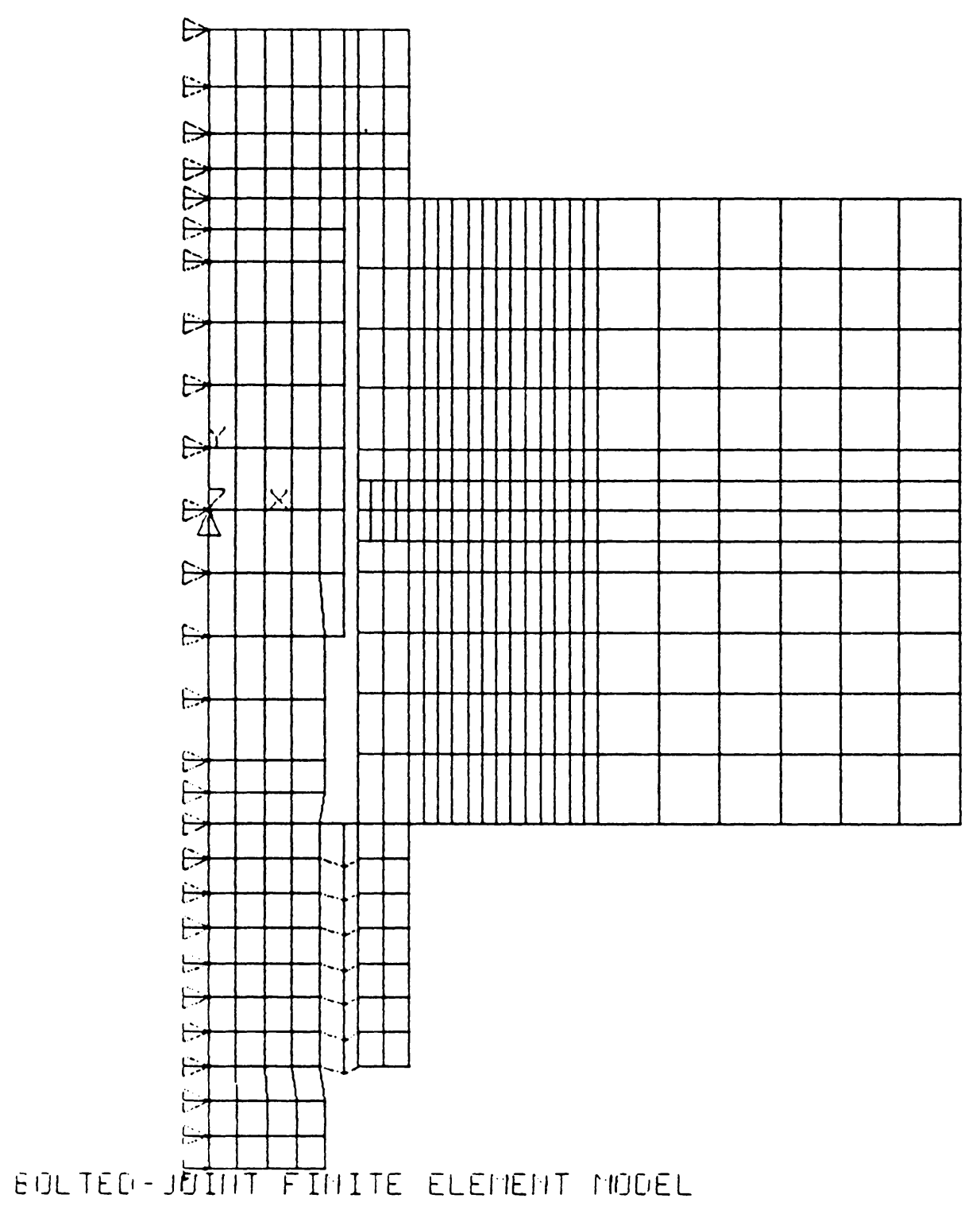

Figure 24. The Bolted-Joint Finite Element Model: Convergence Study No. 1, Mesh No. 3 


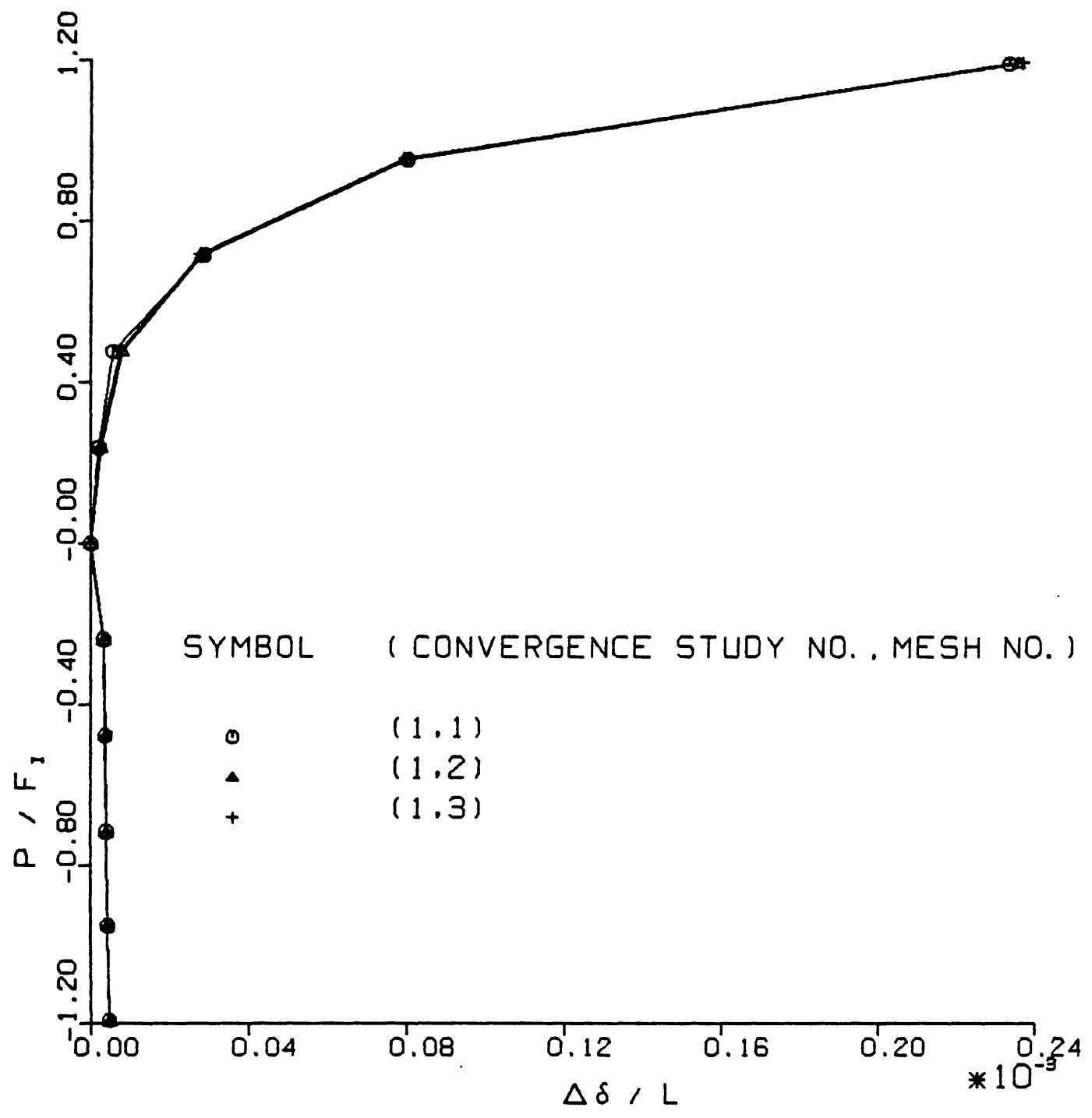

Figure 25. Applied Shear Load versus Bolt Strain: Convergence Study No. 1 


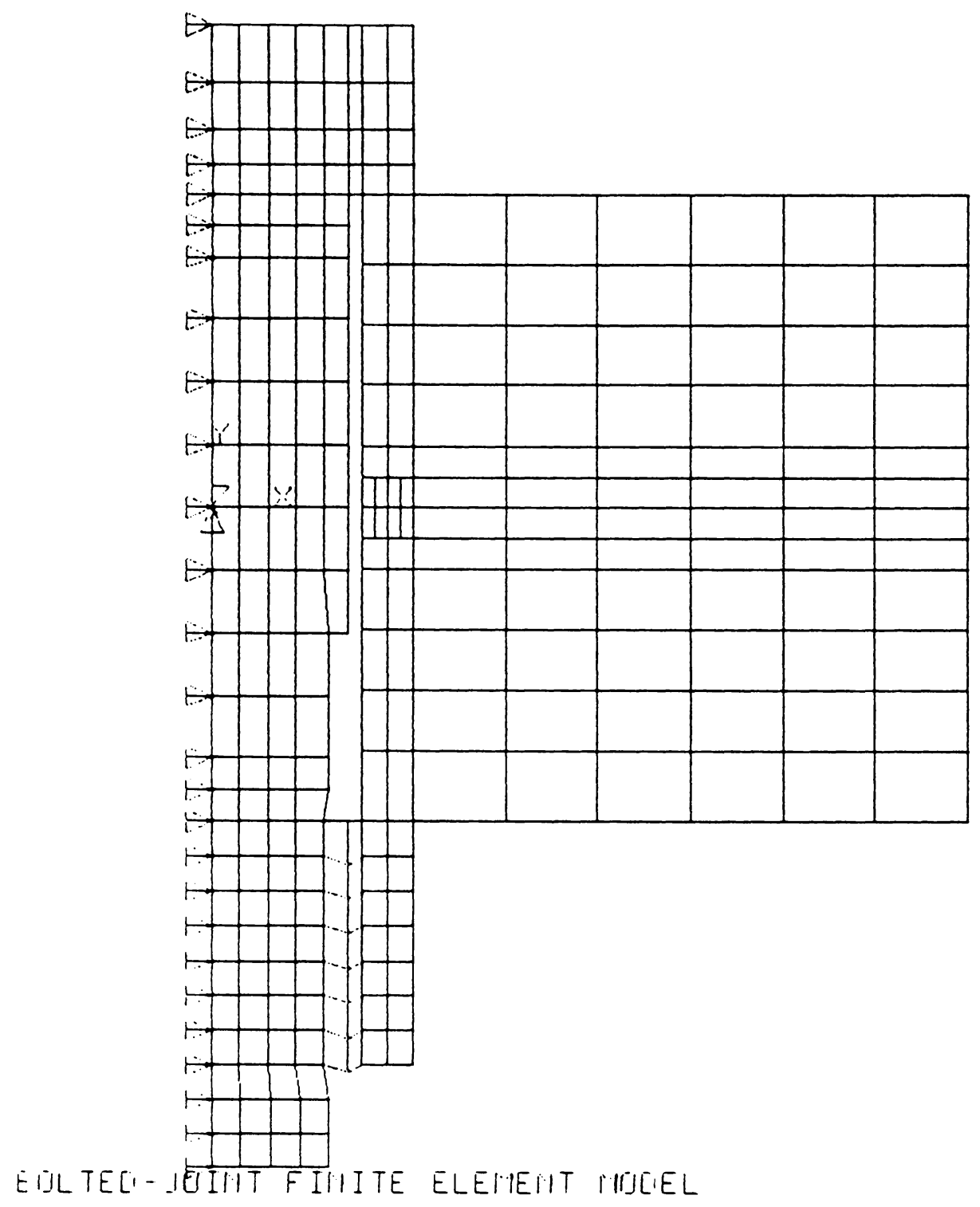

Figure 26. The Bolted-Joint Finite Element Model: Convergence Study No. 2, Mesh No. 1 


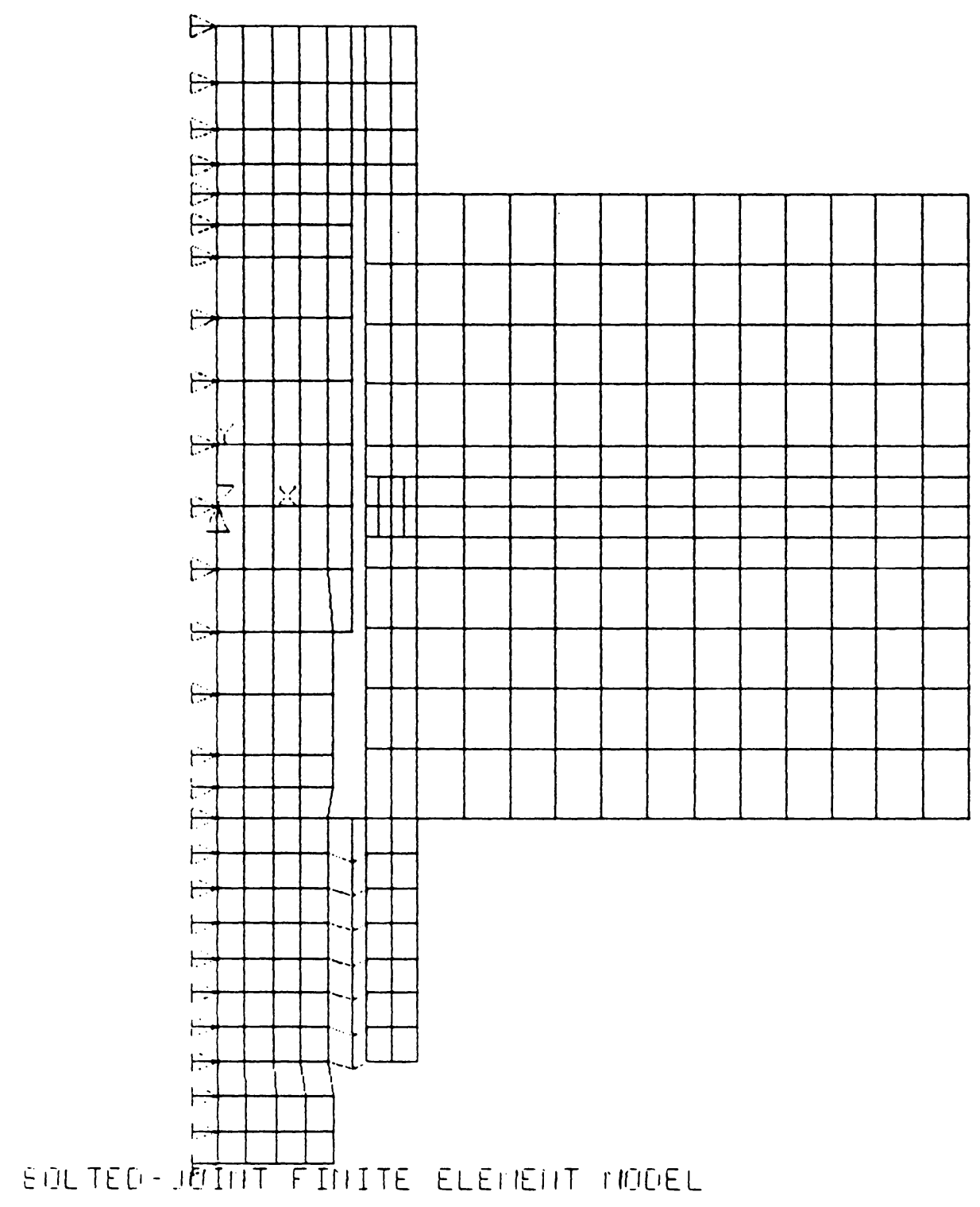

Figure 27. The Bolted-Joint Finite Element Model: Convergence Study No. 2, Mesh No. 2 


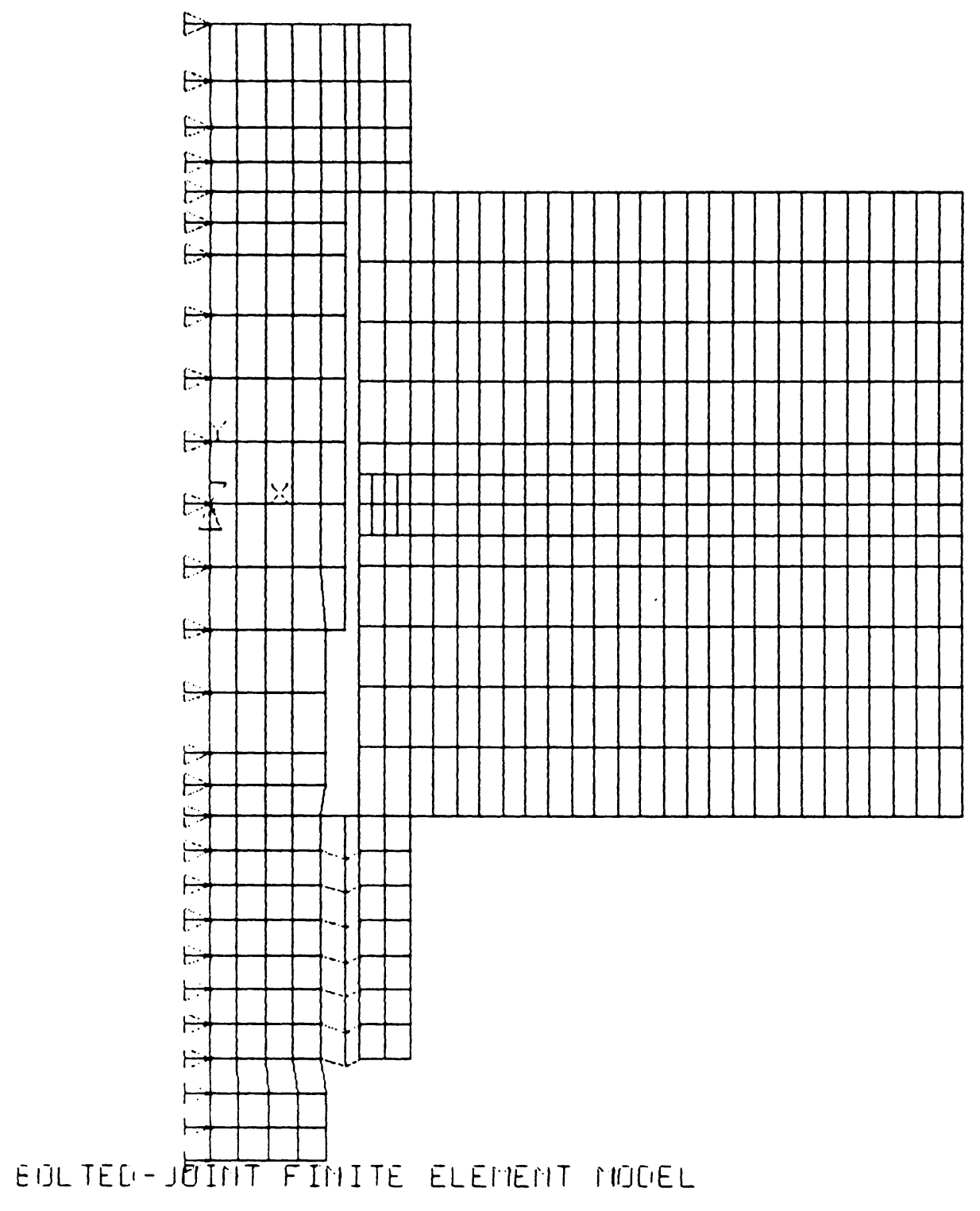

Figure 28. The Bolted-Joint Finite Element Model: Convergence Study No. 2, Mesh No. 3 


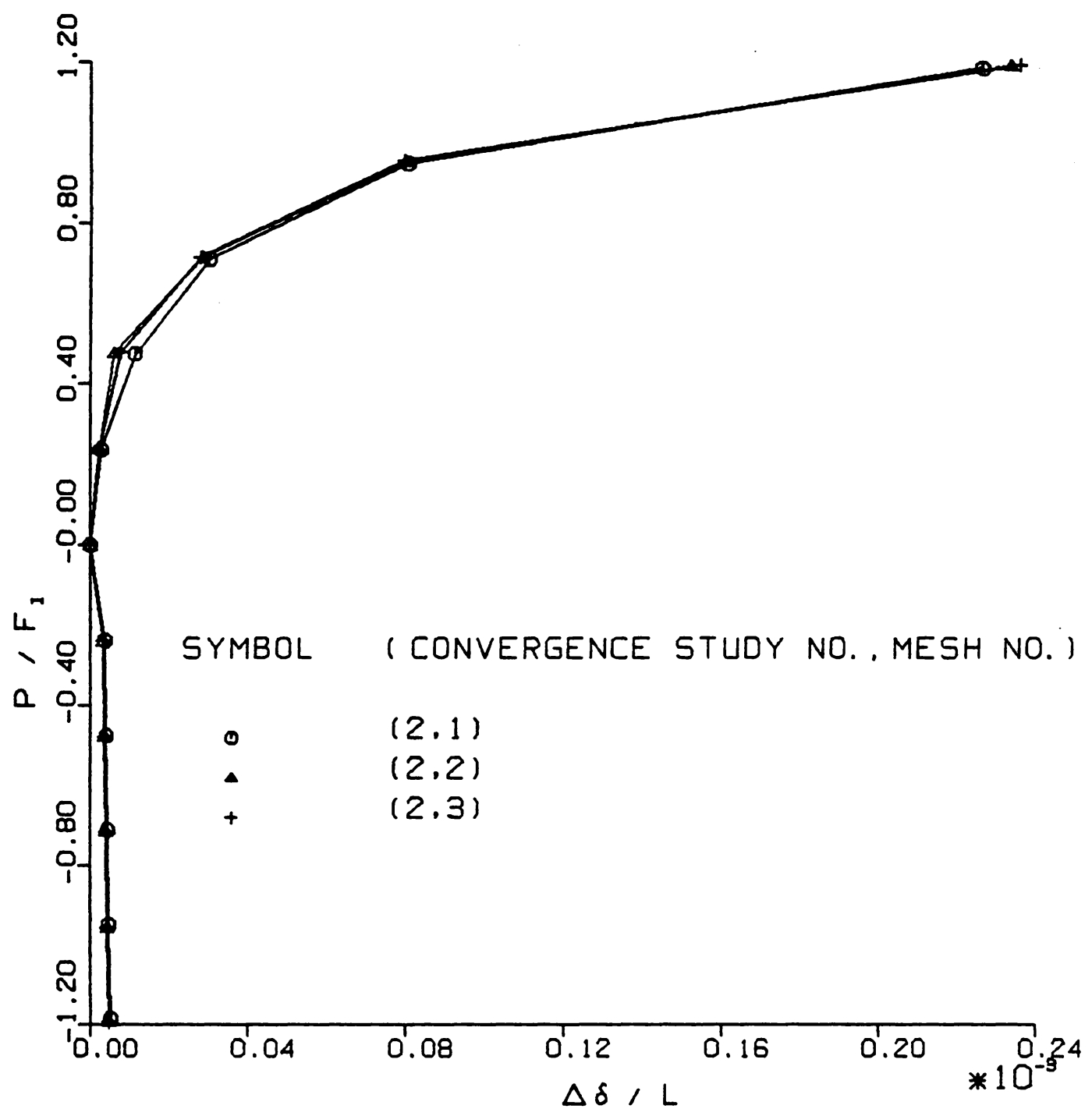

Figure 29. Applied Shear Load versus Bolt Strain: Convergence Study No. 2 


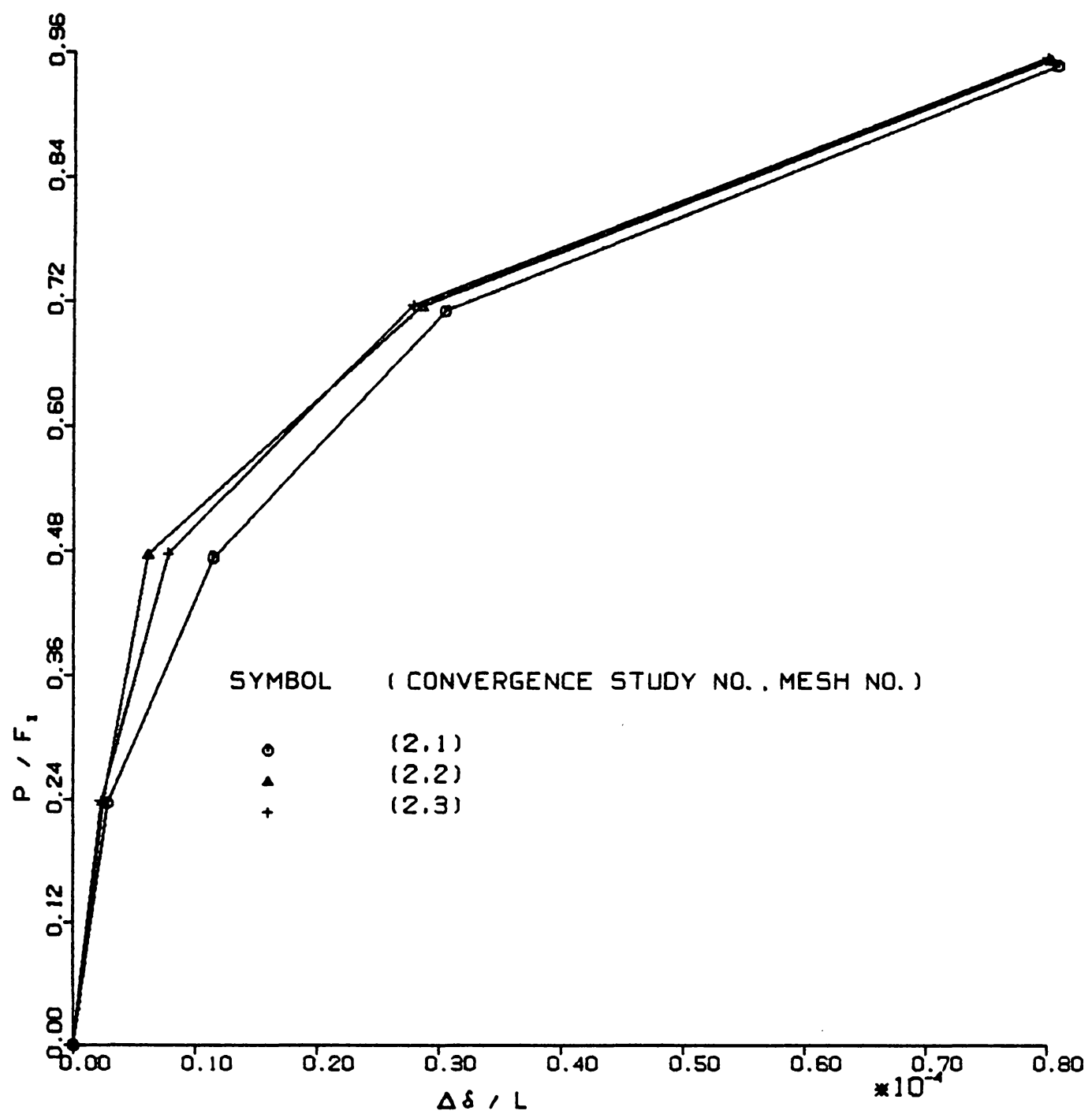

Figure 30. Zoomed Plot of Figure 29: Convergence Study No. 2 
The previous meshes of the first and second convergence study included five-noded transition elements near the plate/plate interface between $r=7.75 \mathrm{~mm}$ and $\mathrm{r}=10.4$ $\mathrm{mm}$. These transition elements were used to increase the number of gap elements at the plate/plate interface in this region. However, some concern arose over the use of these 5-noded transition elements in terms of their compatibility and convergence capabilities. For example, it was not known how ANSYS handled the interelement incompatibility of these five-noded elements.

For this reason a third convergence study was undertaken which examined mesh refinement in the region between the hole radius and the bolt head radius using only fournoded QM6 elements. The converged mesh of Figure 24 on page 62 was used for the remainder of the joint member region. Figures 31 through 33 show the finite element models used. Results are shown in Figure 34. The load-bolt strain curve from the model of Figure 24 on page 62 with the five-noded transition elements is also included in the figure for comparison. Mesh refinement in this region does not appear to have a significant effect on the load-bolt strain curve.

A fourth and final convergence study considered radial mesh refinement in all regions of the bolted-joint model between the thru-hole radius and the edge of the joint. Figures 35 through 38 show the meshes and results of this convergence study. The converged results from the model of Figure 33 are also shown in Figure 38 for comparison. With the exception of the extremely crude mesh of Figure 35, results from this convergence study are in good agreement with the previous converged solutions.

The convergence studies indicate that a converged load-bolt strain solution is obtainable with as little mesh refinement as in Figure 36. However, this mesh has only three gap elements on both the bolt head/plate and nut/plate interfaces and only eleven gap ele- 


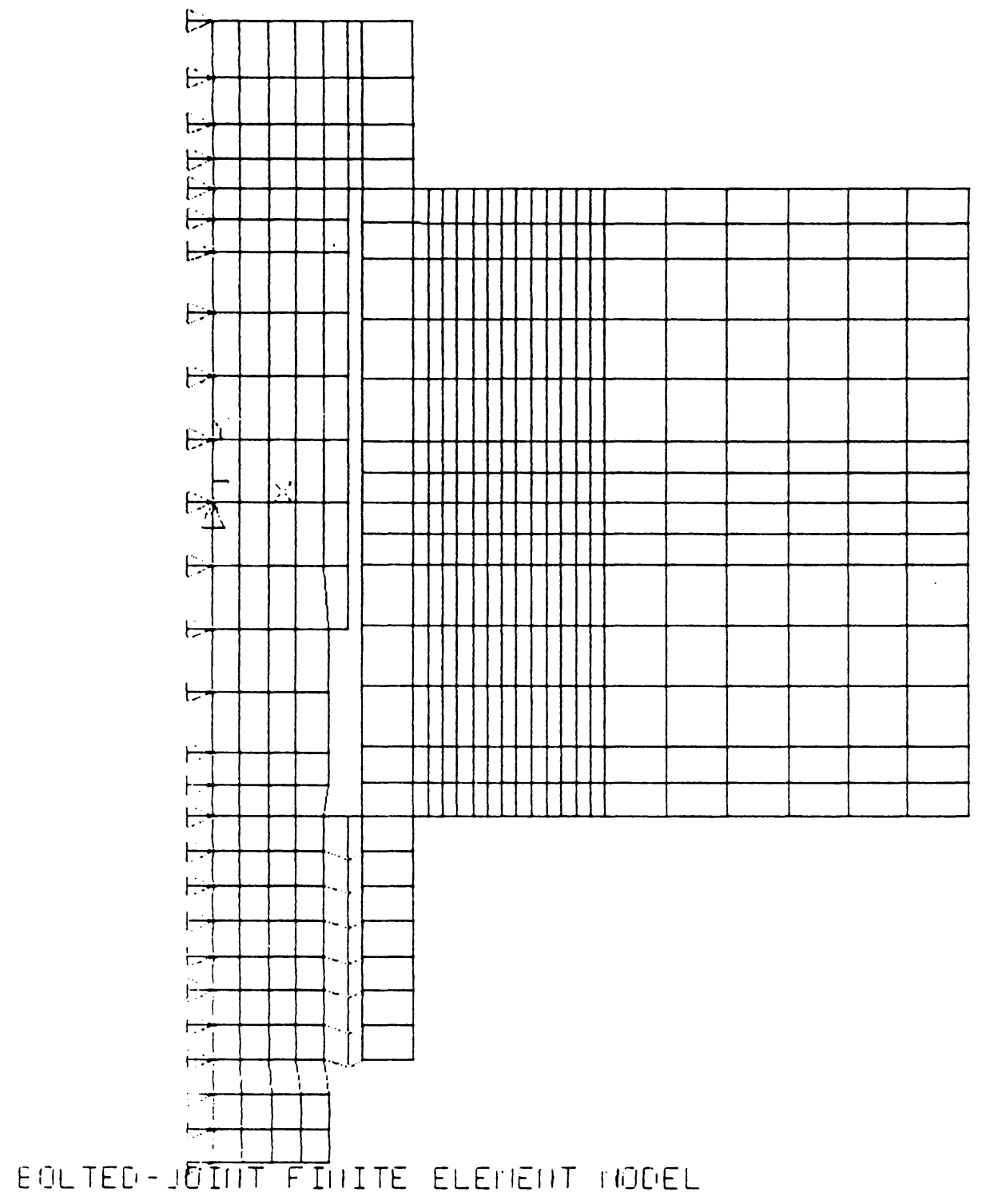

Figure 31. The Bolted-Joint Finite Element Model: Convergence Study No. 3, Mesh No. 1 


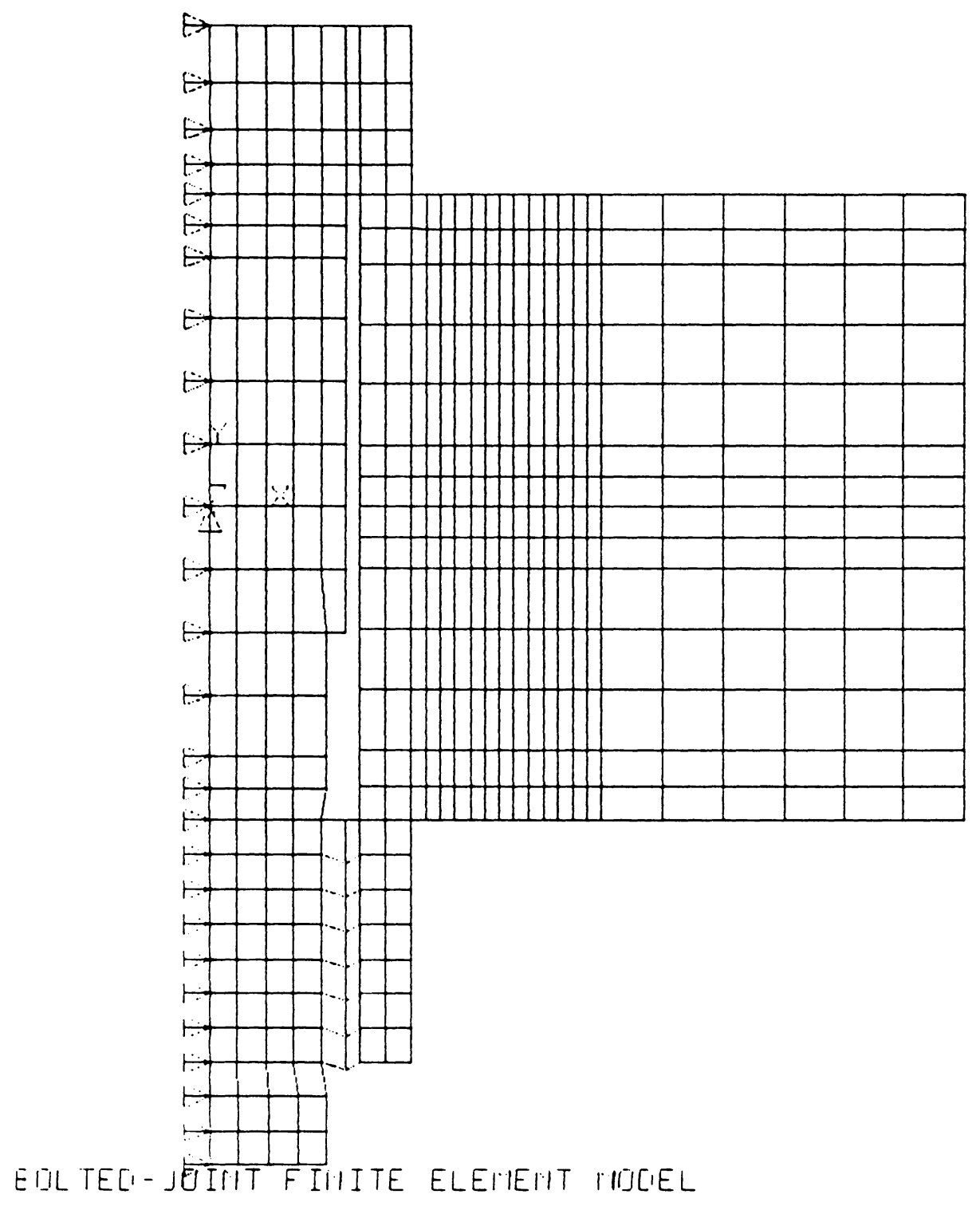

Figure 32. The Bolted-Joint Finite Element Model: Convergence Study No. 3, Mesh No. 2 


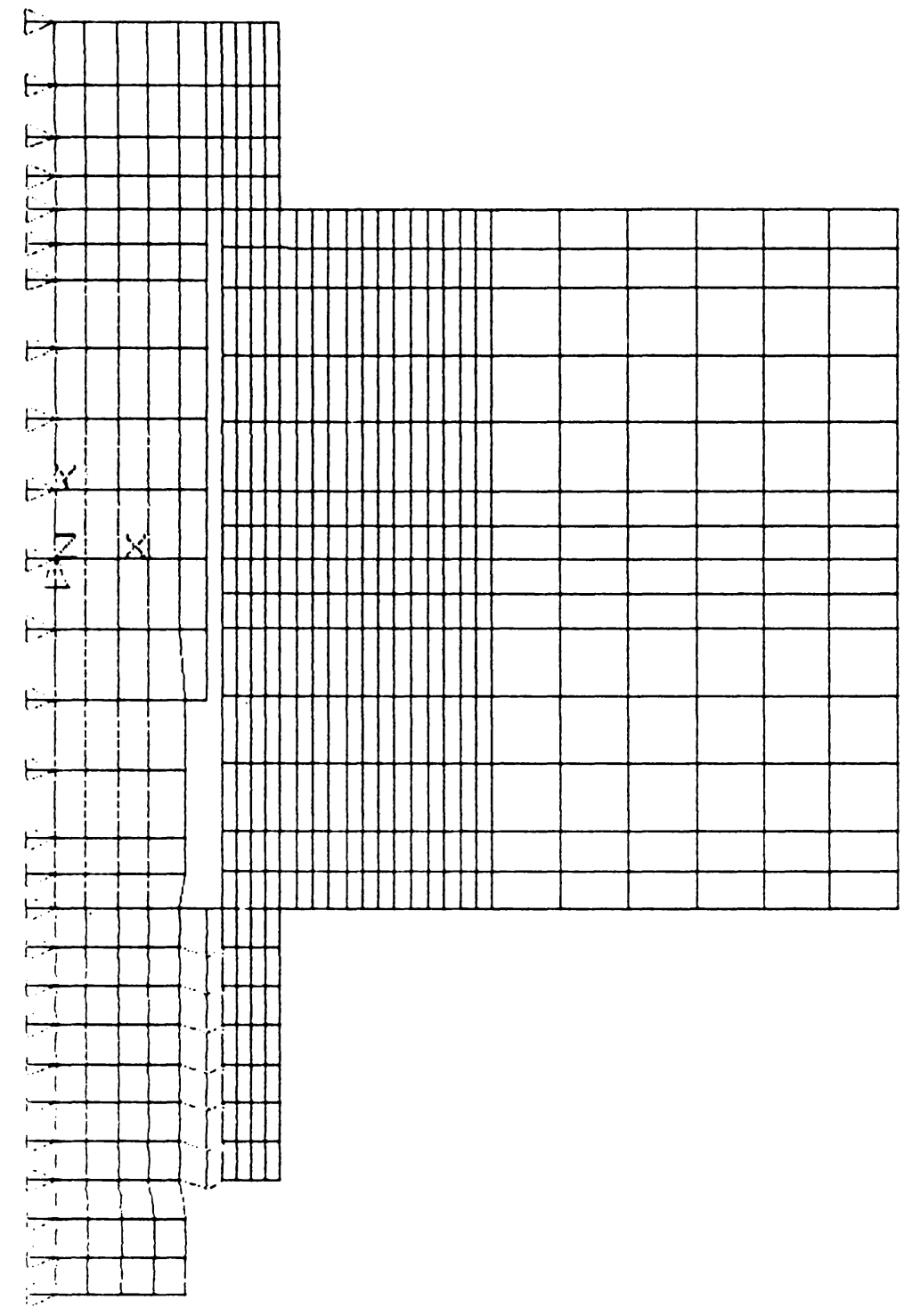

Figure 33. The Bolted-Joint Finite Element Model: Convergence Study No. 3, Mesh No. 3 


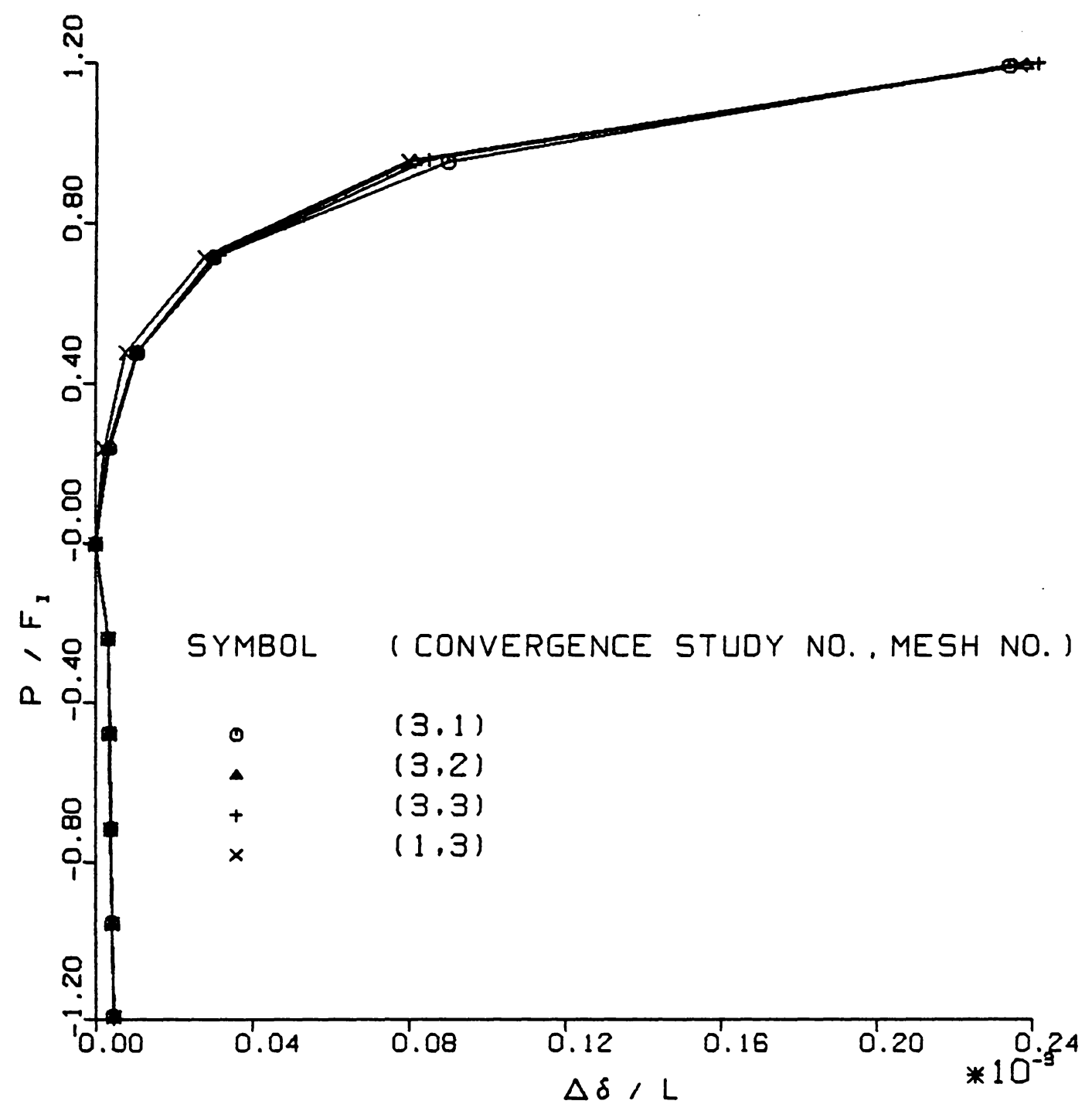

Figure 34. Applied Shear Load versus Bolt Strain: Convergence Study No. 3 


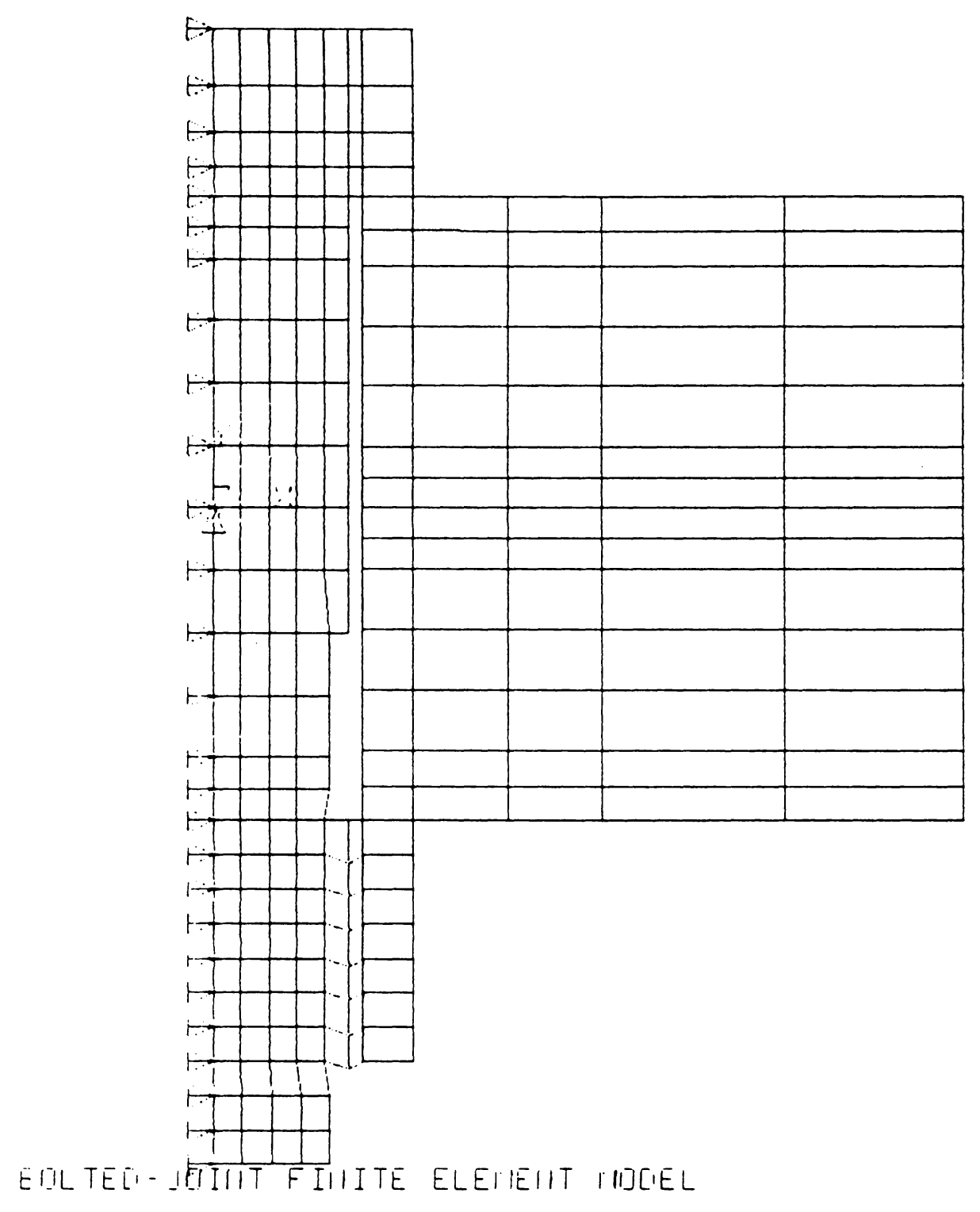

Figure 35. The Bolted-Joint Finite Element Model: Convergence Study No. 4, Mesh No. 1 


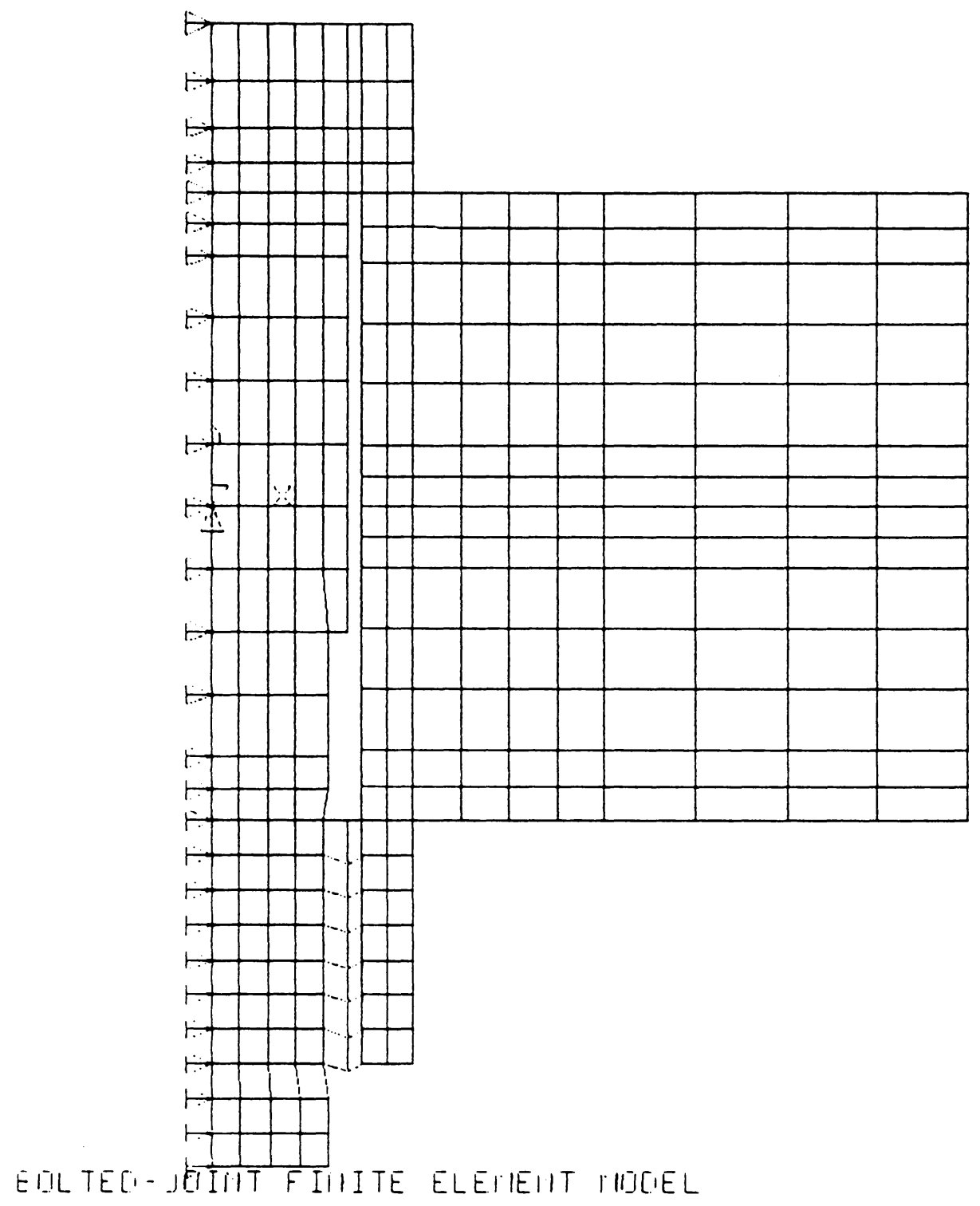

Figure 36. The Bolted-Joint Finite Element Model: Convergence Study No. 4, Mesh No. 2 


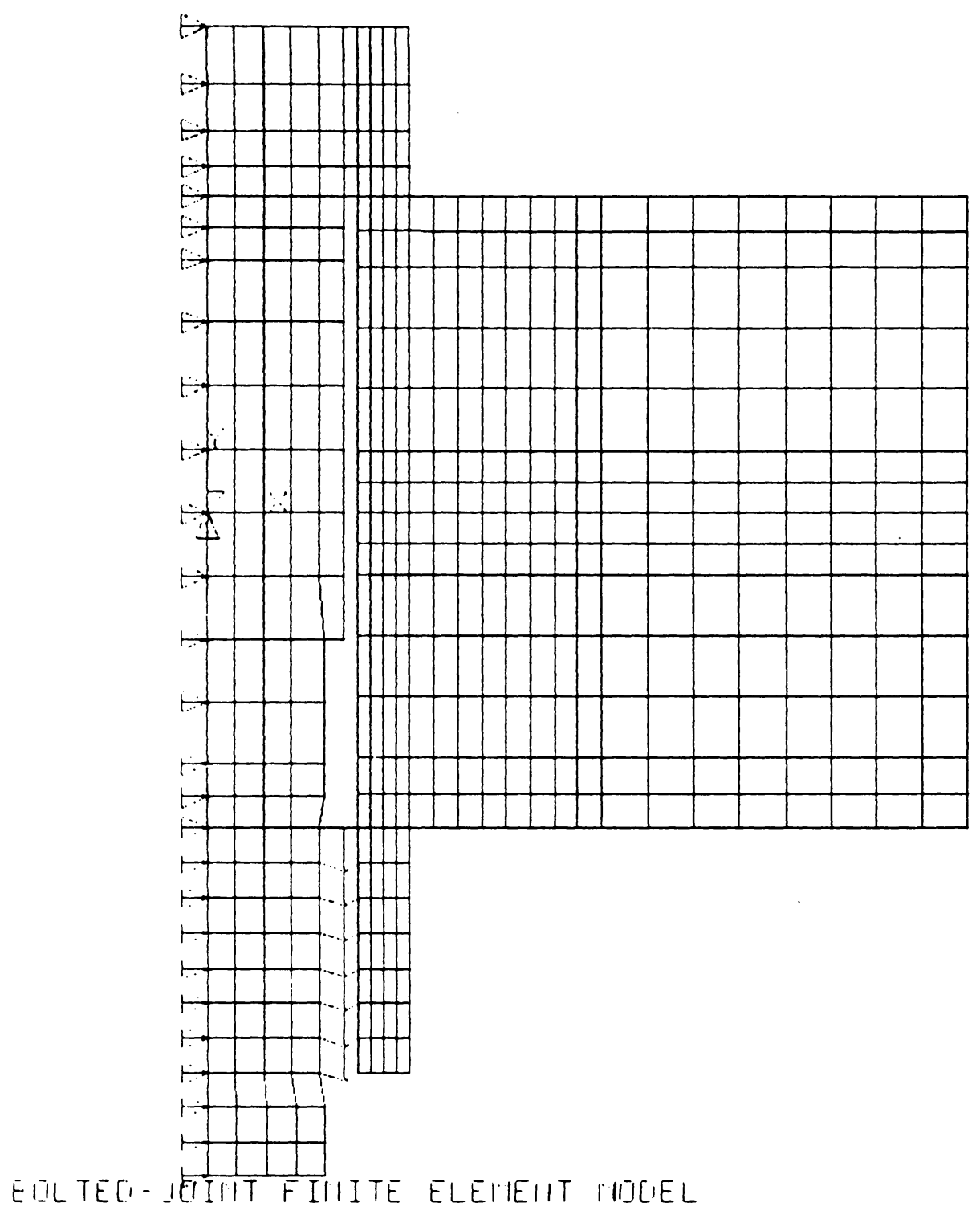

Figure 37. The Bolted-Joint Finite Element Model: Convergence Study No. 4, Mesh No. 3 


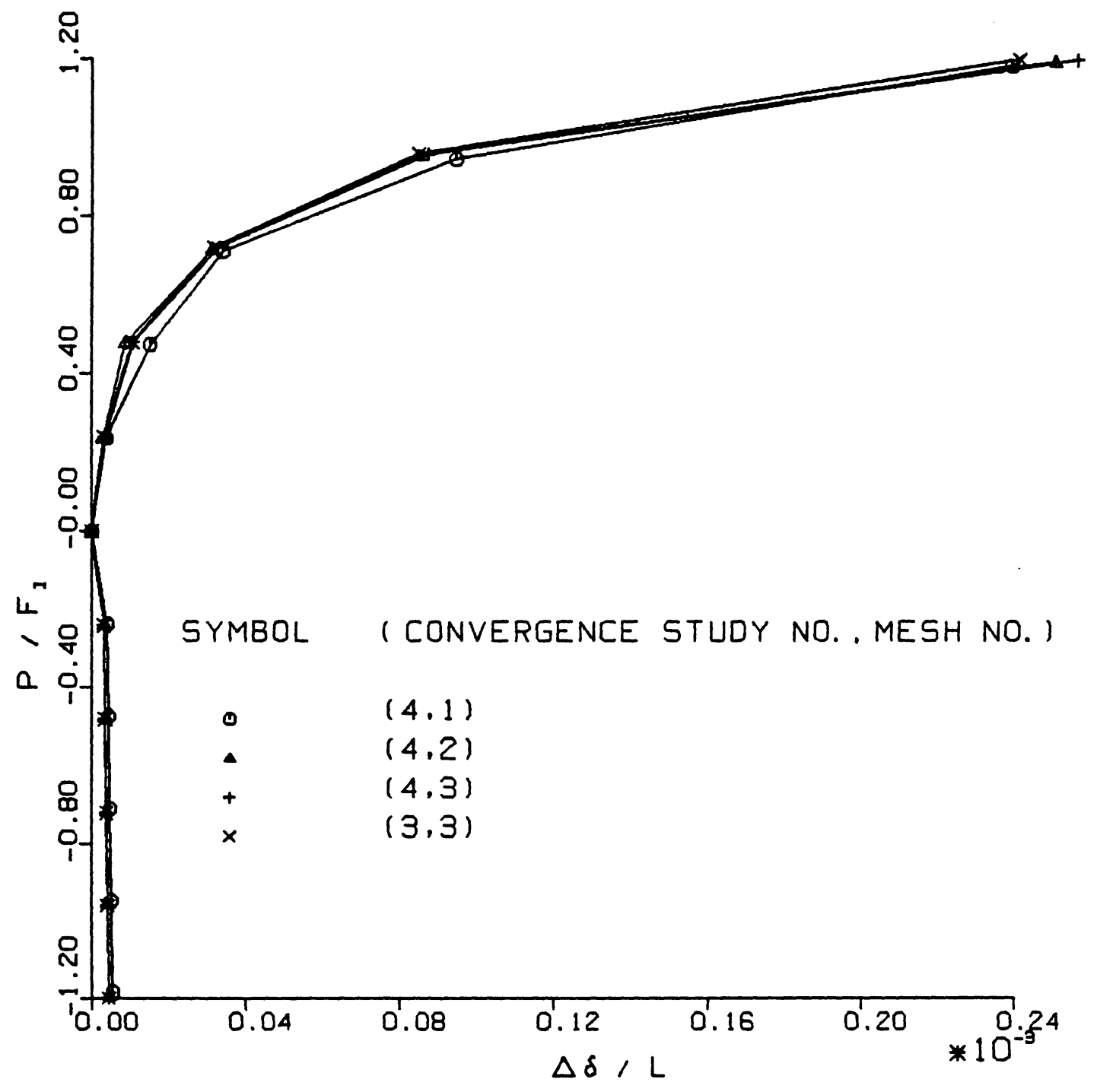

Figure 38. Applied Shear Load versus Bolt Strain: Convergence Study No. 4 
ments modeling the plate/plate interface. Hence, in the interest of obtaining both an accurate load-bolt strain solution and providing accurate pressure distributions along the joint interfaces, the mesh of Figure 33 on page 72 was selected. Most of the results presented in the following chapter will be based on this finite element model.

A linear finite element analysis was performed to assess the accuracy of joint member mesh. A single joint member was modeled with an identical mesh as in the selected bolted-joint model. The joint member was fixed on the inside edge and loaded on the outside edge with a shear load equal to $1.2 F_{i}$. Figure 39 shows the model deformation. The theoretical deflection and rotation of the outside edge is calculated in Appendix B using Roark and Young [33]. Both deflection due to bending and shear were included in the analysis. The finite element model of Figure 39 gave an average edge deflection within $1.85 \%$ of the theoretical value. Using the radial nodal displacements, the finite element results yielded an outside edge rotation within $2.81 \%$ of the theoretical value.

The selected mesh of Figure 33 on page 72 has 534 elements, 605 nodes, and 1182 active degrees of freedom. The wavefront was optimized to yield a maximum wavefront of 70 with a root mean square wavefront of 40 . An eleven load step analysis run used to construct a typical load-bolt strain curve required approximately 54 complete iterations on the formulation and decomposition of the structure stiffness matrix. CPU times on an IBM 4341-2 computer were generally $2000-2500$ seconds for an eleven load step analysis run. 


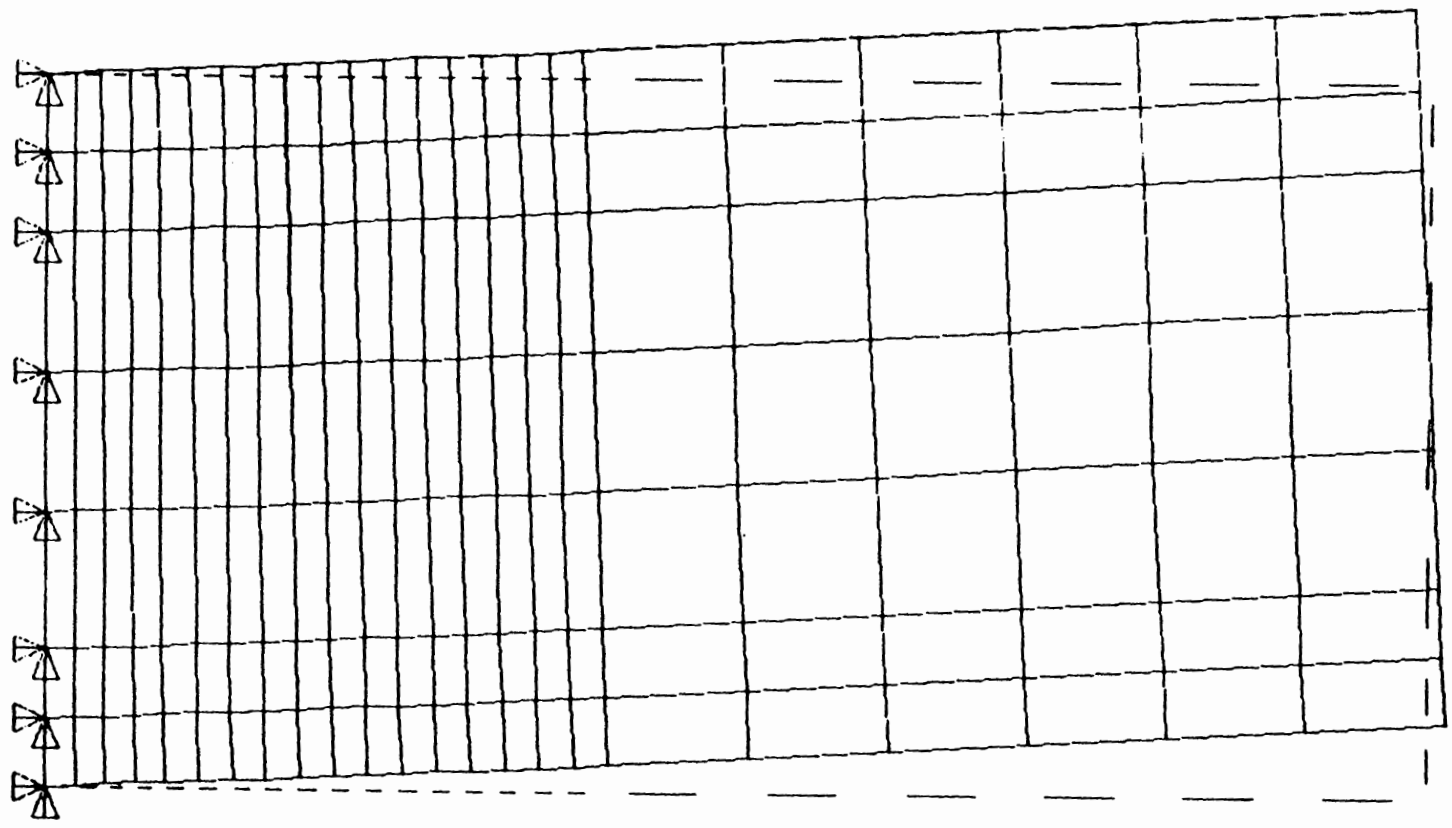

Figure 39. Deformation of Joint Member Fixed at Inside Edge with Shear Load on Outside Edge 


\section{Chapter 5}

\section{Results and Discussion}

\subsection{The Bolted-Joint under Preload}

Figure 40 shows the preload deformation of the bolted-joint model. The joint preload, achieved by thermal contraction of the bolt, is $3.93\left(10^{4}\right) \mathrm{N}$, yielding a bolt preload stress $\sigma_{i}=342 . \mathrm{MPa}$. Displacements in the figure have been exaggerated by a factor of 98.7 for illustrative purposes. A free-edge boundary condition was employed on the outer diameter of the joint model. Because this is an axisymmetric analysis, a pinned or fixed boundary condition was not considered realistic. A rotational-stiffness boundary condition could possibly be justified in view of the surrounding structure. However, the preload deformation results shows little outer edge rotation. Furthermore, a rotational stiffness-boundary condition is difficult to implement in a finite element model that does 


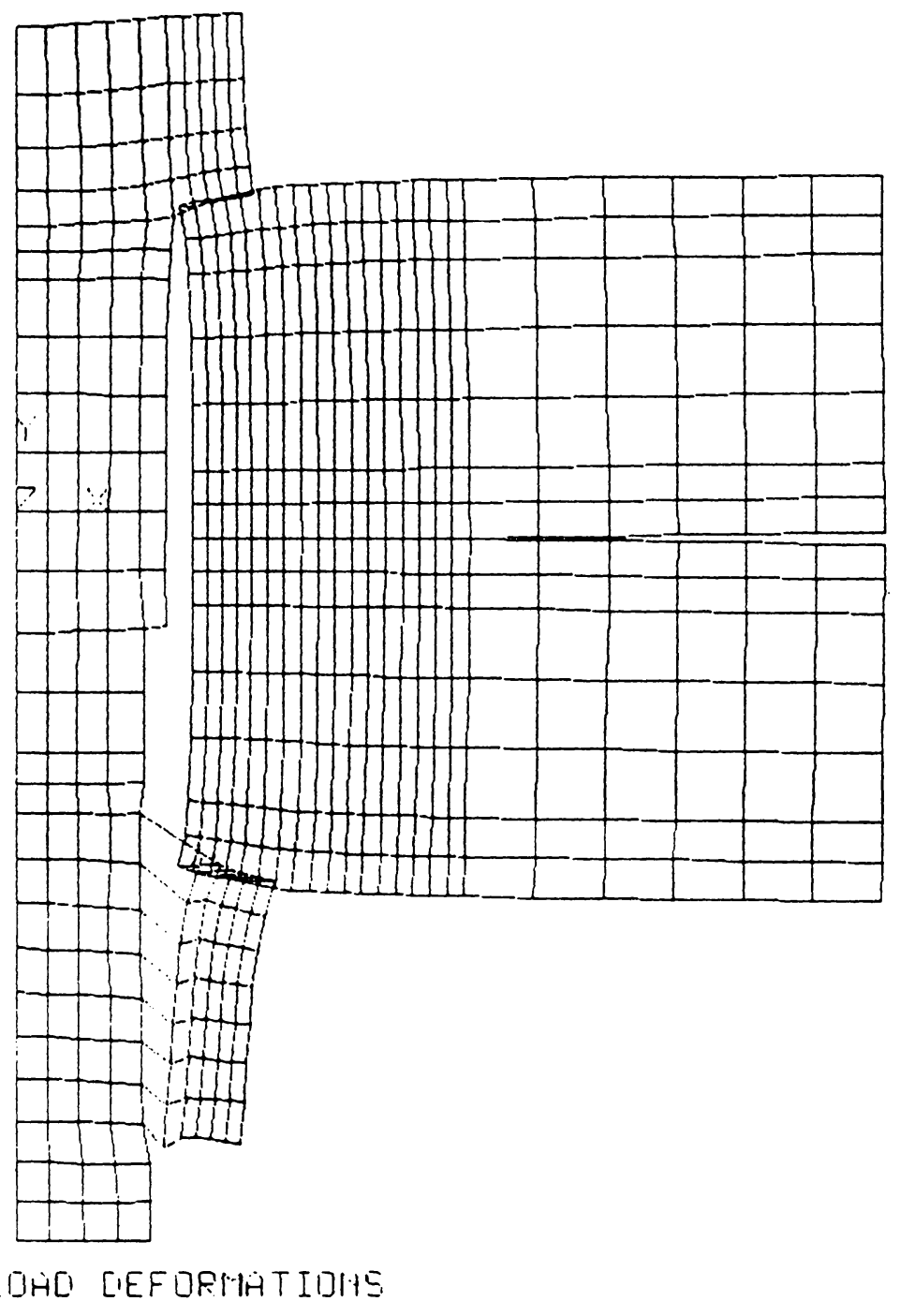

Figure 40. Preload Deformation of the Bolted-Joint Model 
not have rotational degrees of freedom. Knowledge of the surrounding structure is needed before a rotational stiffness value can be calculated.

There are several key observations to note from Figure 40 on page 81 . First, the joint preload has resulted in separation of the joint members for radii greater than or equal to $r=2.5 r_{H}$, where $r_{H}$ is the bolt hole radius. Hence, compressive loads on the joint members at the nut/plate interface and bolt head/plate interface have resulted in interface separation outside of a given radius. A similar effect will be seen for the case of applied compressive loads at the joint model edge. This separation effect has been well documented in the literature through both experimental data and finite element analysis (Ito et al. [12], Bradley [34], Gould and Mikic [20], Ziada and Abd El Latif [22]).

Figure 40 on page 81 also shows a significant difference between the bolt head and nut deformation. Due to the special "thread" elements, a large radial nut expansion is seen compared to the bolt head. The perceived overlapping of element boundaries is a result of the deformation exaggeration. The plot also reveals significant bending in both the bolt head and nut.

The preload pressure distribution at the joint interface is shown in Figure 41 . The interfacial pressure has been nondimensionalized by $Q$, the mean interfacial pressure at the bolt head/plate interface. Results are in good agreement with the finite element results of Reference [20] for a bolted joint of similar proportions. The shape and size of the preload compression region can easily be seen by the axial stress contours of Figure 42. Note that the orientation of the axial stress contours in the plates are mostly vertical, indicating a high axial stress gradient in the radial direction. Recall that the linear bolted-joint theory presented in Chapter 2 assumed a uniform axial stress in the radial direction within the compressed joint member region to calculate the compressed mem- 


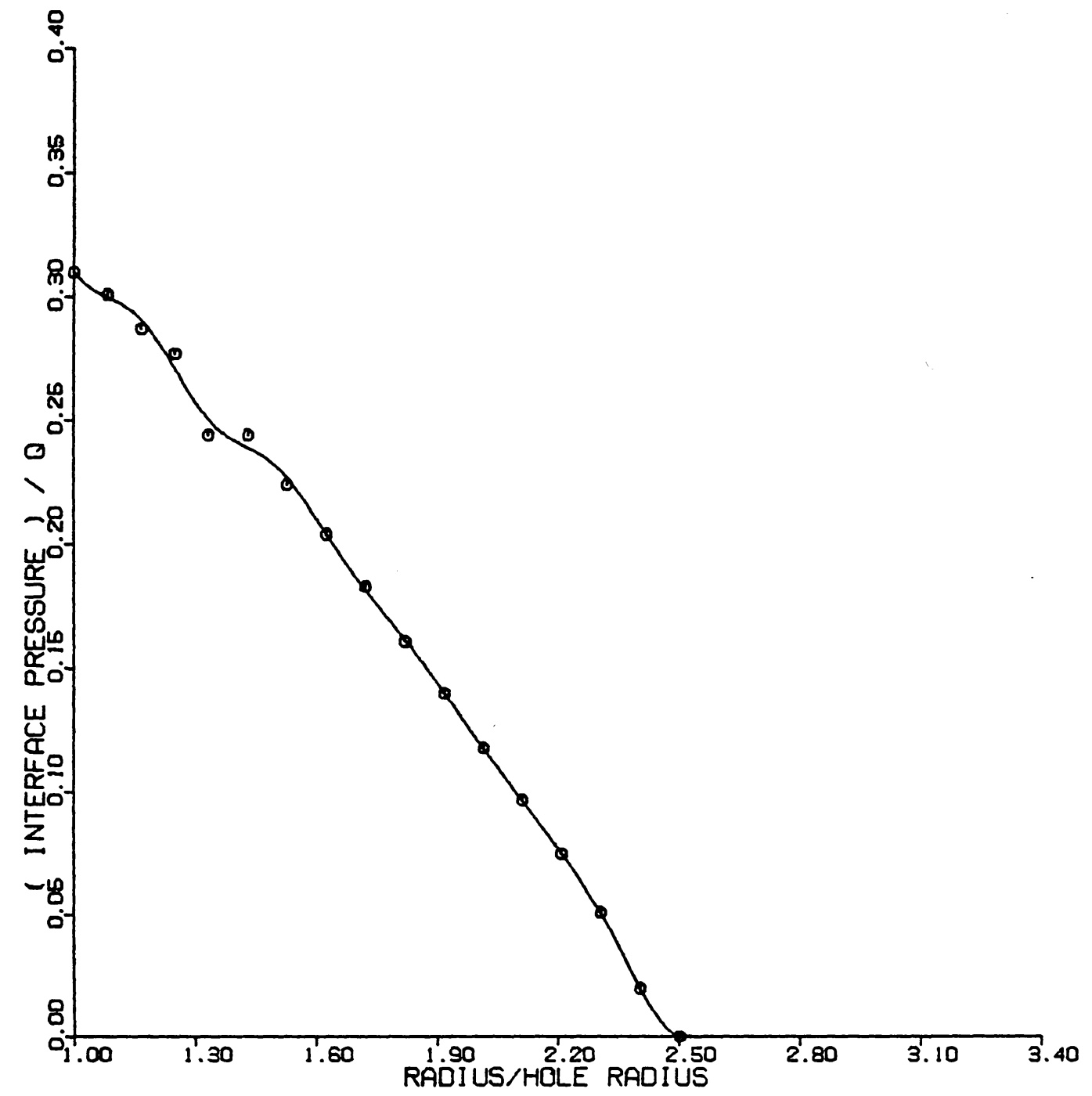

Figure 41. Plate/Plate Interface Pressure Distribution Due to Preload 


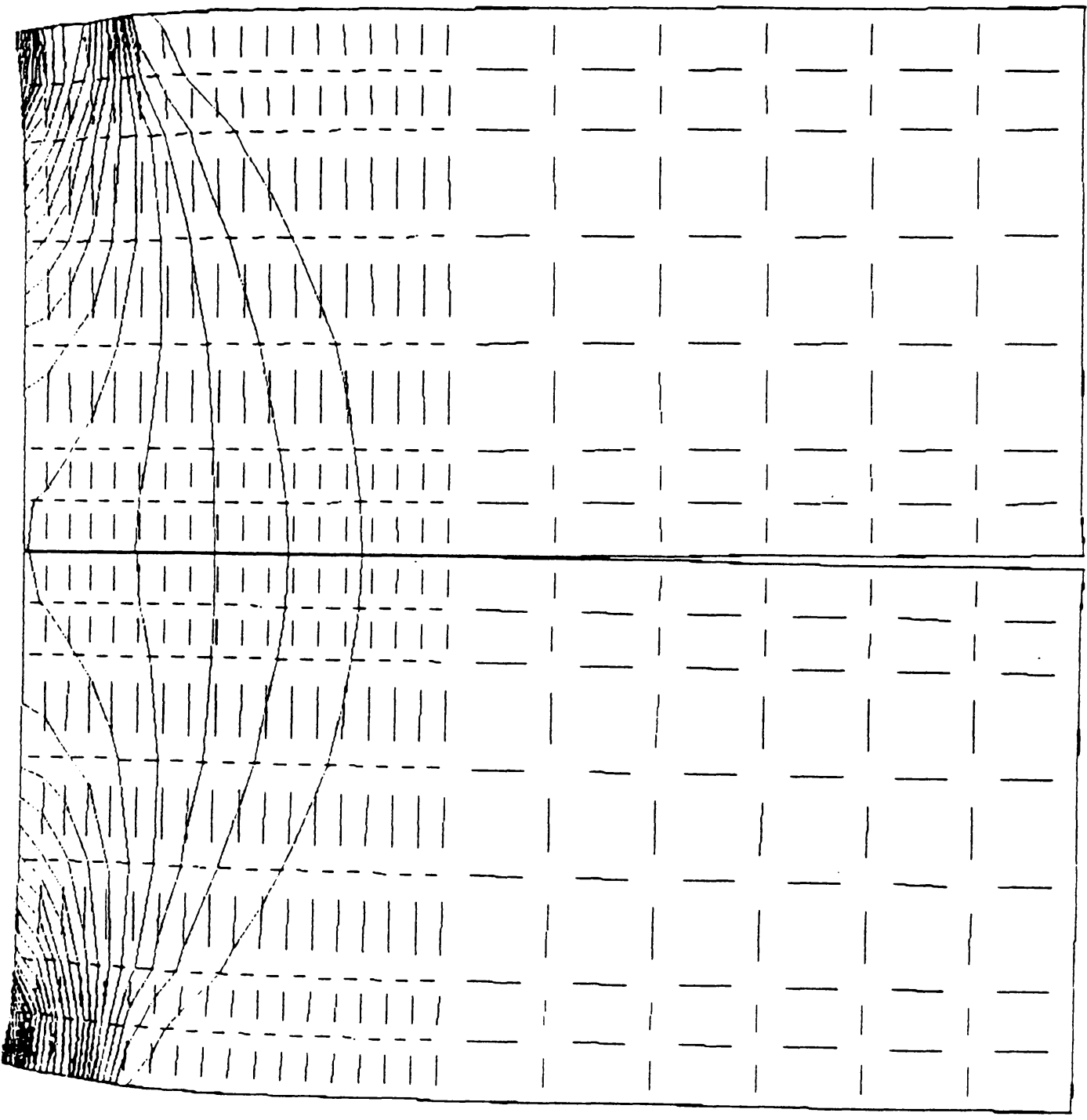

Figure 42. Preload Axial Stress Distribution in the Joint Members 
ber stiffness $k_{m}$. If this were the case, then the axial stress contours in this region of Figure 42 would be horizontal, not mostly vertical. Stiffness calculations based on this assumption are not correct.

\subsection{The Preloaded Bolted Joint under Applied Shear Loads}

Figures 43 through 45 show the deformations of the bolted-joint model under various applied shear loads at the edge of joint. Based on the good agreement between finite element results and Goodier's experimental data on radial nut expansion shown in Figure 17 on page 44 , a coefficient of friction of 0.1 was used at the bolt head/plate, nut/plate, and plate/plate interfaces. Figure 43 and Figure 44 have applied tensile shear loads of $0.48 \mathrm{~F}_{\mathrm{i}}$ and $1.20 \mathrm{~F}_{\mathrm{i}}$, whereas Figure 45 has an applied compressive shear load of $-0.48 \mathrm{~F}_{\mathrm{i}}$. The nodal loads in the $\mathrm{y}$ (axial direction) were calculated from the total applied shear load $P$ using Eq. (3.9). It can be seen in Figure 44 that the $1.2 F_{i}$ applied tensile shear load has resulted in complete joint interface separation. Surprisingly, the joint members show less bending then one might initially think for this situation. One is tempted to attributed this lack of bending to the finite element discretization error which generally results in stiff models. However, the finite element mesh used to model the joint member was shown in Chapter 4 to give excellent agreement with sheardeformable, thick-plate theory. The lack of bending in the joint members is due to the nature of the boundary condition on the joint members. Gap elements along the bolt head/plate and nut/plate interface allow the plates to rotate about the edge of the bolt head and nut once complete joint separation has occurred. 


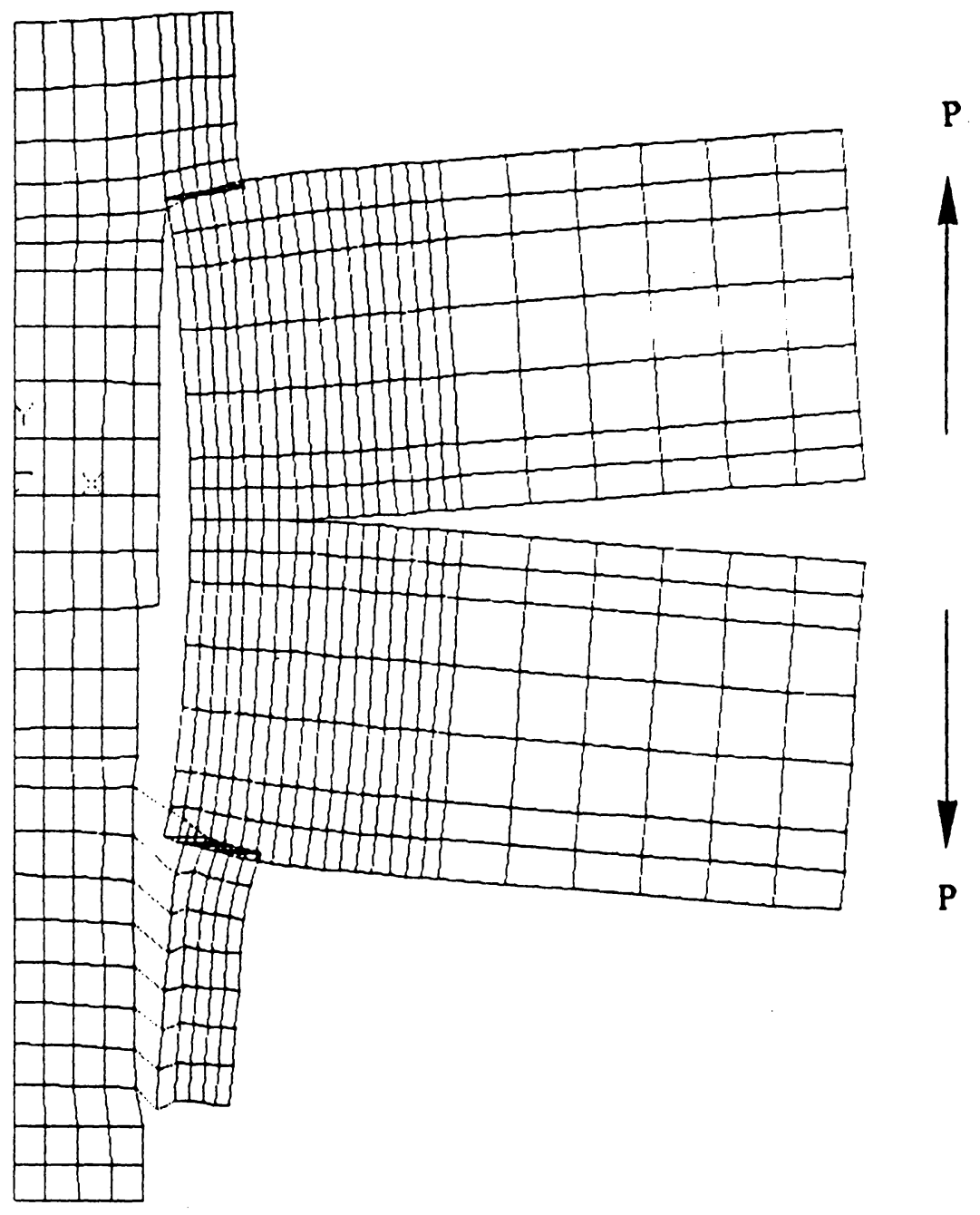

Figure 43. Deformation of the Bolted Joint: $\mu=0.1, P=0.48 \mathrm{~F}_{\mathrm{i}}$ 


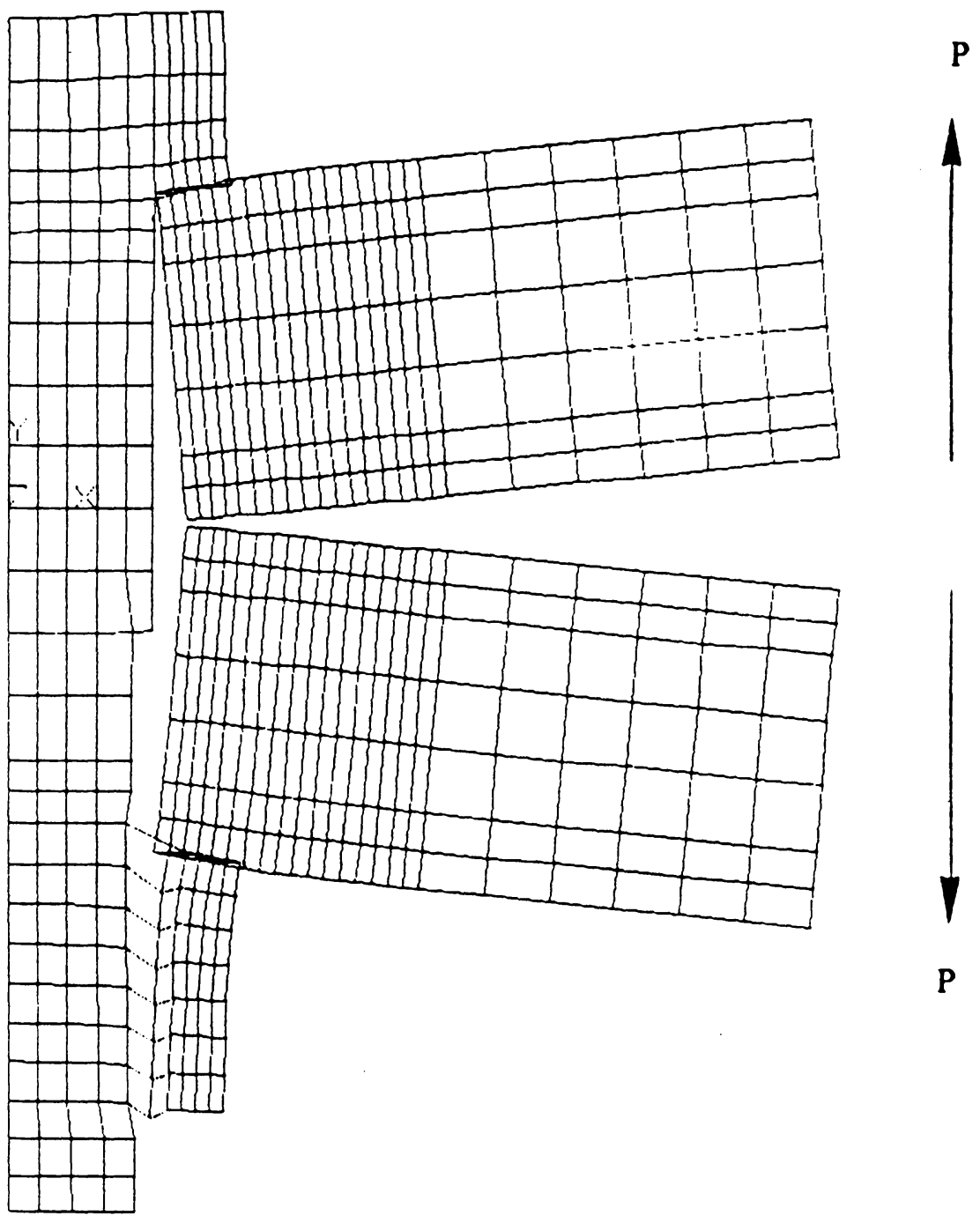

Figure 44. Deformation of the Bolted Joint: $\mu=0.1, P=1.20 \mathrm{~F}_{\mathrm{i}}$ 


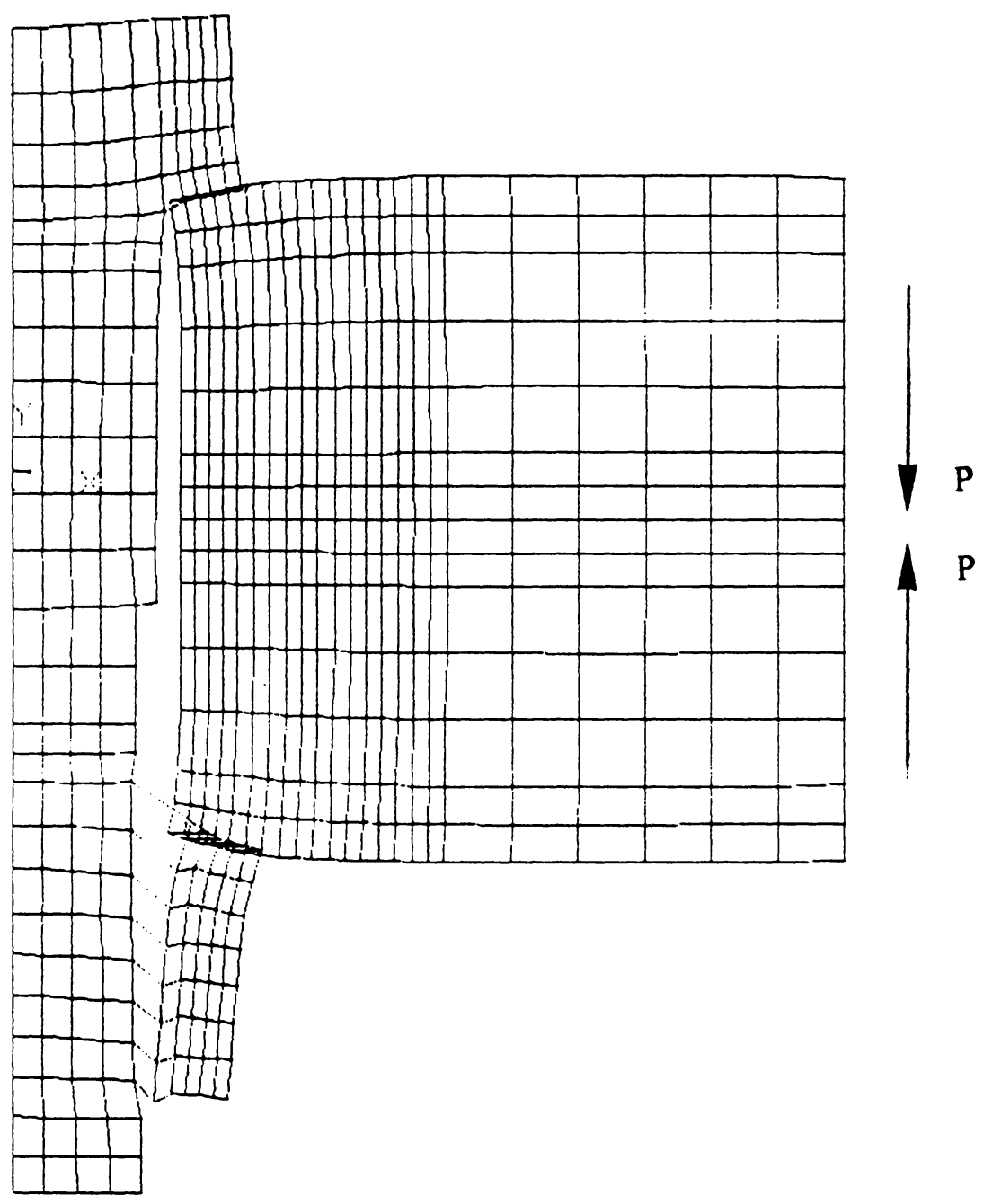

Figure 45. Deformation of the Bolted Joint: $\mu=0.1, P=-0.48 F_{i}$ 
Figure 46 shows the applied shear load versus bolt strain from an eleven load step finite analysis using the model of Figure 33 on page 72 . All bolt strains are relative to the bolt preload strain. The bolt strains were computed by dividing the axial extension (or contraction) between the bolt head and nut by the bolt length between the bolt head and nut. Since the bolt has different sectional areas, this quantity is not the true bolt strain but rather an average bolt strain over the bolt head-to-nut length. All subsequent bolt-strain references refer to the average bolt strain. The slope of this curve is proportional to the overall joint stiffness $k_{T}$. For compressive (negative) applied loads, the figure reveals an interesting effect. Applied compressive shear loads at the joint-model edge result in small, but tensile, bolt strains relative to the bolt preload strain. This effect can be viewed as a prying effect. Note from Figure 45 on page 88 that the compressive applied shear load has resulted in additional contact along the member/member interface. Compressive reaction forces exerted on the top joint member by the bottom joint member in this contact region are equilibrated by the applied compressive shear load and additional compressive loads exerted on the top member by the bolt head. The same argument holds for the forces exerted on the bottom joint member by the top joint member and the nut. Since the compressive forces underneath the bolt head and nut have increased, the final result is a small increase in bolt strain relative to preload for negative applied moments. Yet, the maximum tensile bolt strain due to an applied compressive load of $-1.2 F_{i}$ is only $4.54\left(10^{-6}\right)$, whereas the maximum tensile bolt strain due to an applied tensile load of $1.2 \mathrm{~F}_{\mathrm{i}}$ is $2.57\left(10^{-4}\right)$.

One can see from Figure 46 that the joint stiffness is highly dependent on the magnitude of the applied tensile shear load. For example, a $0.24 \mathrm{~F}_{\mathrm{i}}$ load increment applied at preload yields a bolt strain increment of $3.34\left(10^{-6}\right)$, whereas the same load increment applied to the joint at $0.96 \mathrm{~F}_{\mathrm{i}}$ yields a bolt strain increment of $1.69\left(10^{-4}\right)$. Hence, over 


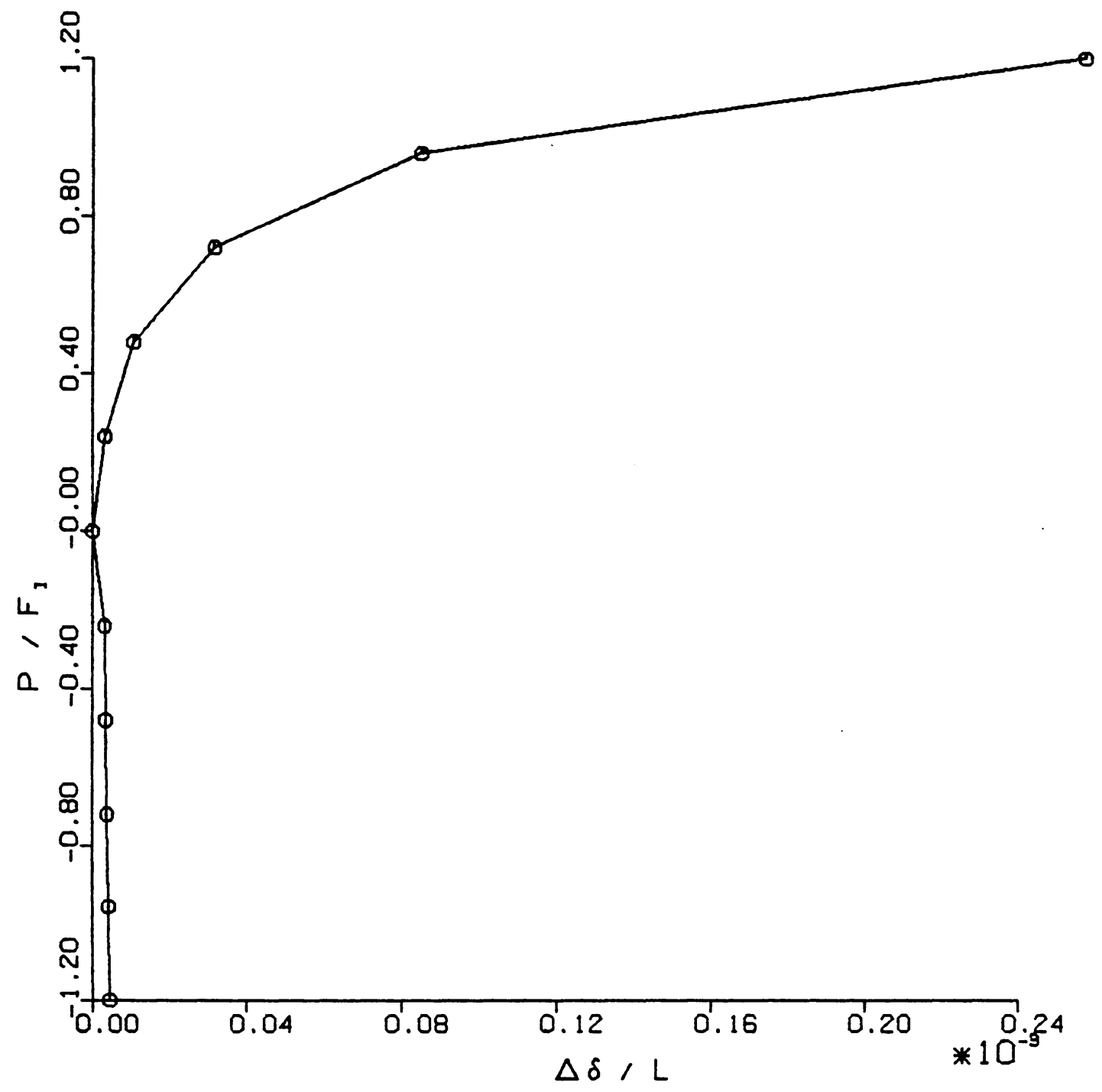

Figure 46. Applied Shear Load versus Bolt Strain: $\mu=0.1$ 
a fifty-fold decrease in joint stiffness is seen between the joint preload state and the 0.96 $\mathrm{F}_{\mathrm{i}}$ applied-tensile-load state!

Deformation plots of Figure 43 on page 86 and Figure 44 on page 87 provide important clues as to why there is such a tremendous dependence of joint stiffness on applied tensile shear loads. The deflection of the joint members under applied tensile shear loads is seen to consist mainly of two types of motion: rotation and axial translation. When small tensile shear loads are applied to the joint edge, the top and bottom joint members undergo very little axial translation. Instead each joint member rotates about some circumferential line on the bolt-head or nut-bearing surface. The rotation of the joint members enables the interface between the joint members to undergo more separation then if the joint members were undergoing pure bending and axial translation. On the other hand, rotation of the joint members increases compression along the joint members' interface near the inner edge of the joint members which tends to counteract the compression relief due to separation of the joint members' interface. As the applied tensile loads are increased, rotation of the joint members induces less compression relief because of the reduced size of the compressed member region. In other words, the end-of-contact point along the interface between the joint members has moved radially inward, thereby reducing the distance from end-of-contact point to the pivot points of the joint member. Rotation and bending effects become less signficant at these tensile levels in terms of relieving joint compression. Hence, a greater fraction of the applied tensile shear loads are transferred to the bolt through the bolt head and nut bearing surfaces. Of course, this is a heuristic attempt to explain a complex, three-dimensional nonlinear elastic behavior. 


\subsection{The Preloaded Bolted Joint under Applied}

\section{Moment-Simulating Loads}

Two separate load cases were analyzed to study the effect of moment loading. In both cases applied radial moments at the outer joint edge were simulated using nodal radial forces. The procedure is outlined in Chapter 3. In the first load case only momentsimulating loads were applied to the model. No shear loads were applied. The bolted-

joint deformations due to applied moments of $0.41 \mathrm{M}_{\mathrm{REF}}$ and $-0.41 \mathrm{M}_{\mathrm{REF}}$ are shown in Figure 47 and Figure 48, respectively. Positive applied moments are defined as moments that bend the joint members away from each other at the joint edge. The reference moment, $\mathrm{M}_{\mathrm{REF}}$, was defined as the radial line moment at the inner edge of an annular plate, fixed on the inside diameter and shear loaded on the outside diameter. The joint member geometry was used for the plate geometry. The applied shear load was set to 1.2 $\mathrm{F}_{\mathrm{i}}$, the maximum applied shear load considered in these analyses. Details of the calculations of the reference moment are given in Appendix A.

Figure 49 shows the relationship between the applied moment and the bolt strain relative to the preload strain. Positive applied moments result in a decrease in the bolt strain relative to preload. In contrast, negative applied moments increase the bolt strain. The deformation plots of Figures 47 and 48 are helpful in understanding this behavior. As seen from Figure 47, bending in the plate due to the positive applied moment has resulted in increased interfacial separation between the joint members. Since there are no applied shear loads, the compression relief has resulted in a decrease in the forces exerted 


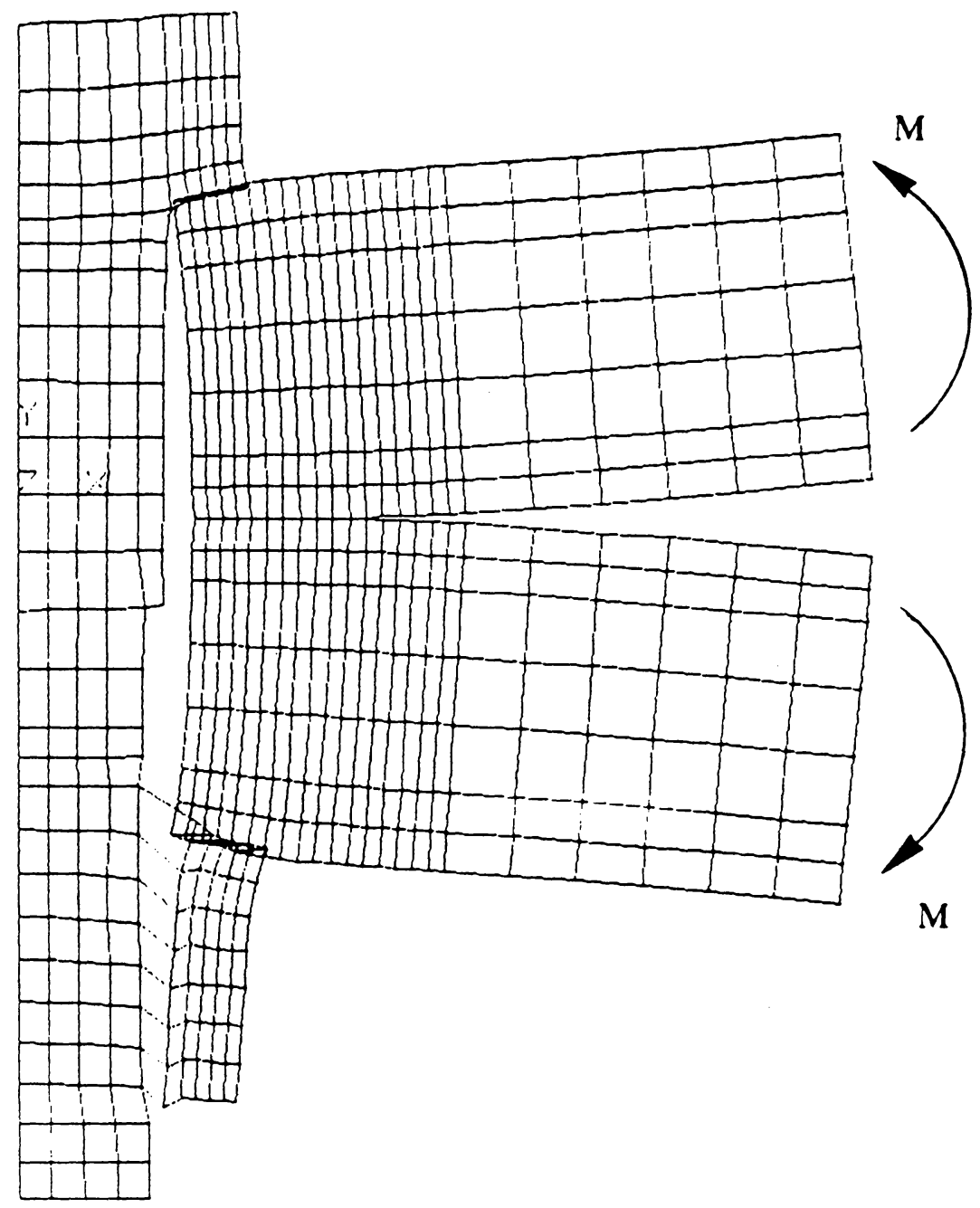

Figure 47. Deformation due to Applied Moment: $M=0.41 M_{\text {REF }}$ 


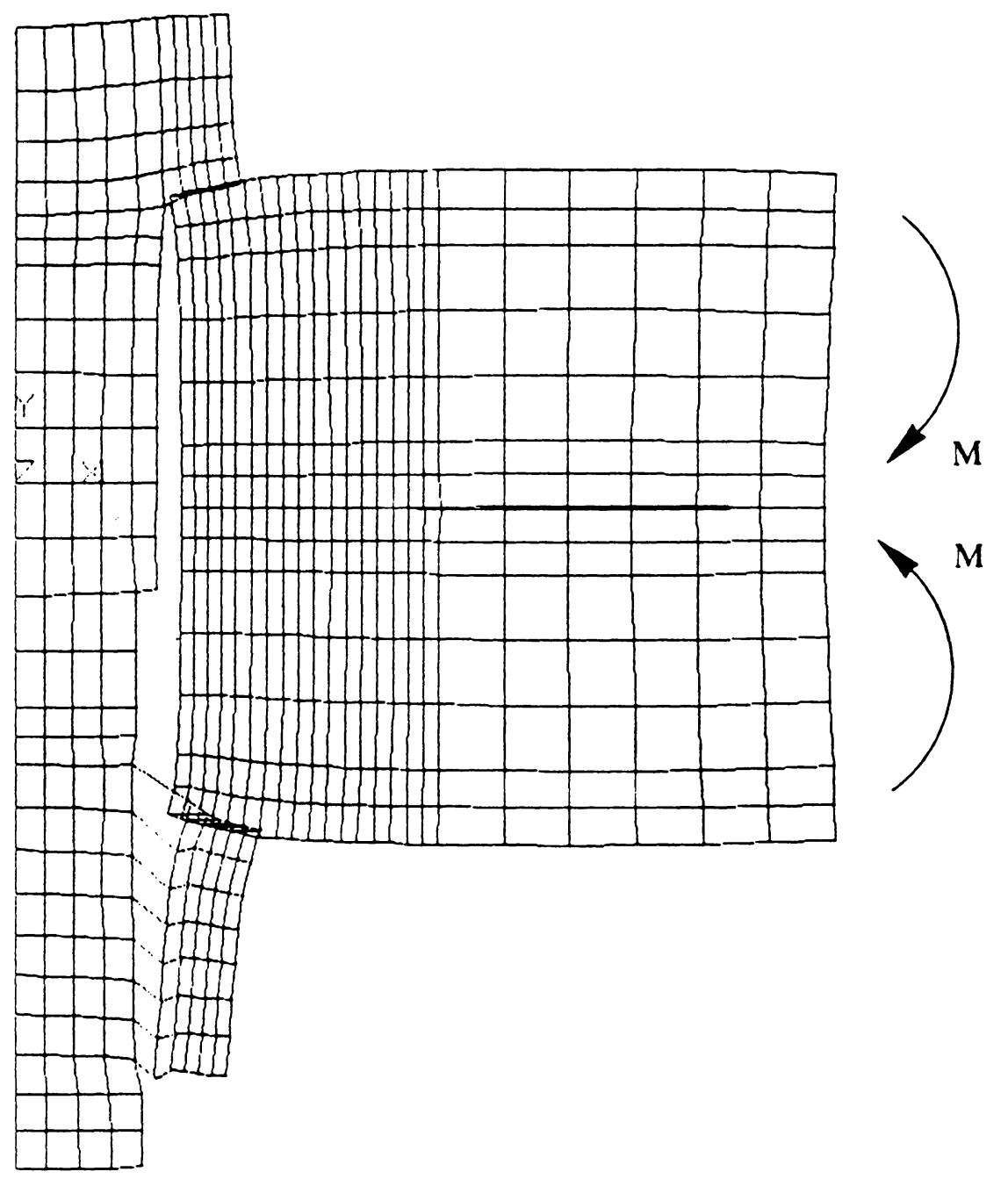

Figure 48. Deformation due to Applied Moment: $M=-0.41 \mathrm{M}_{\text {REF }}$ 


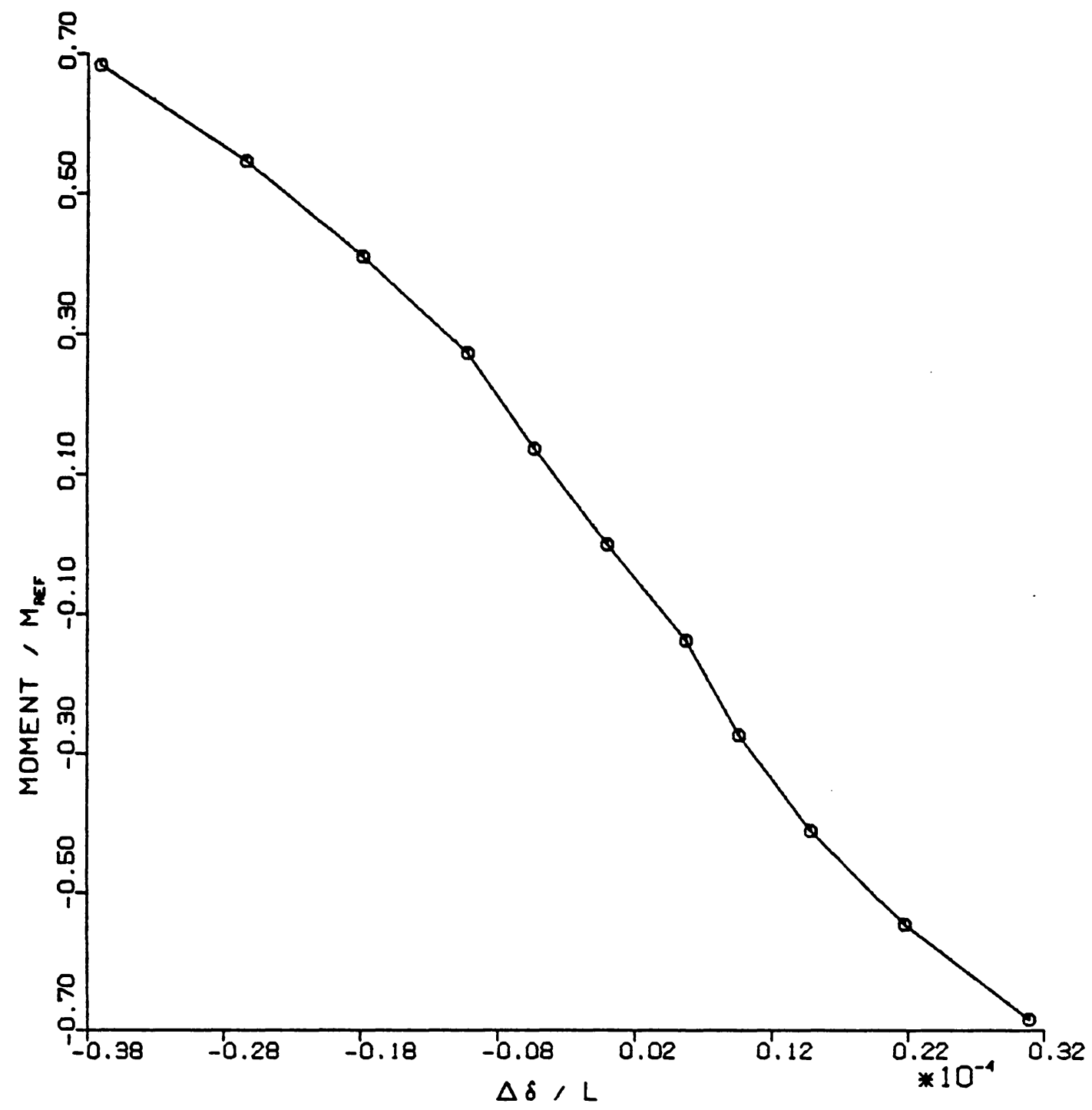

Figure 49. Applied Moment versus Bolt Strain: $\mu=0.1$ 
by the joint members on the bolt head and nut. Consequently, a decrease in bolt strain relative to the preload bolt strain is seen for positive applied moments.

The increase in bolt strain relative to preload for negative applied moments can be understood using Figure 48 on page 94 . Note that the bending moment has resulted in additional contact along the member/member interface on the outer edge of the joint. The net result is a prying effect, similar to the prying effect seen for compressive applied shear loads on the joint edge. However, since there are no compressive applied shear loads in this case, all of the additional reaction forces generated in the new contact region must be balanced by additional compressive forces exerted by the bolt head and nut on the joint members. Thus, for a given prying-action-generated reaction force, there will be more prying exerted on the bolt for negative applied moments versus negative applied shear loads.

Note that Figure 49 on page 95 is relatively linear compared to Figure 46 on page 90 . This fact seems to indicate that bending in the joint members due to an applied shear load only is not the major source of nonlinearity in the joint stiffness seen in Figure 46 on page 90. Rotation of the joint members, not bending, is considered to be the major factor influencing the nonlinear joint stiffness. The moment results presented here suggest that any bending action due to an applied tensile shear load will help reduce the portion of the applied tensile shear load carried by the bolt.

The second analysis of moment loading considered the case of combined positive shear and moment loading. The magnitude of the applied moment was proportional to the magnitude of the shear load as described in Chapter 3. The results are compared with the shear-loading-only results in Figure 50. The combined positive moment and shear loading induces an extremely high, slightly negative joint stiffness near the preload state. 


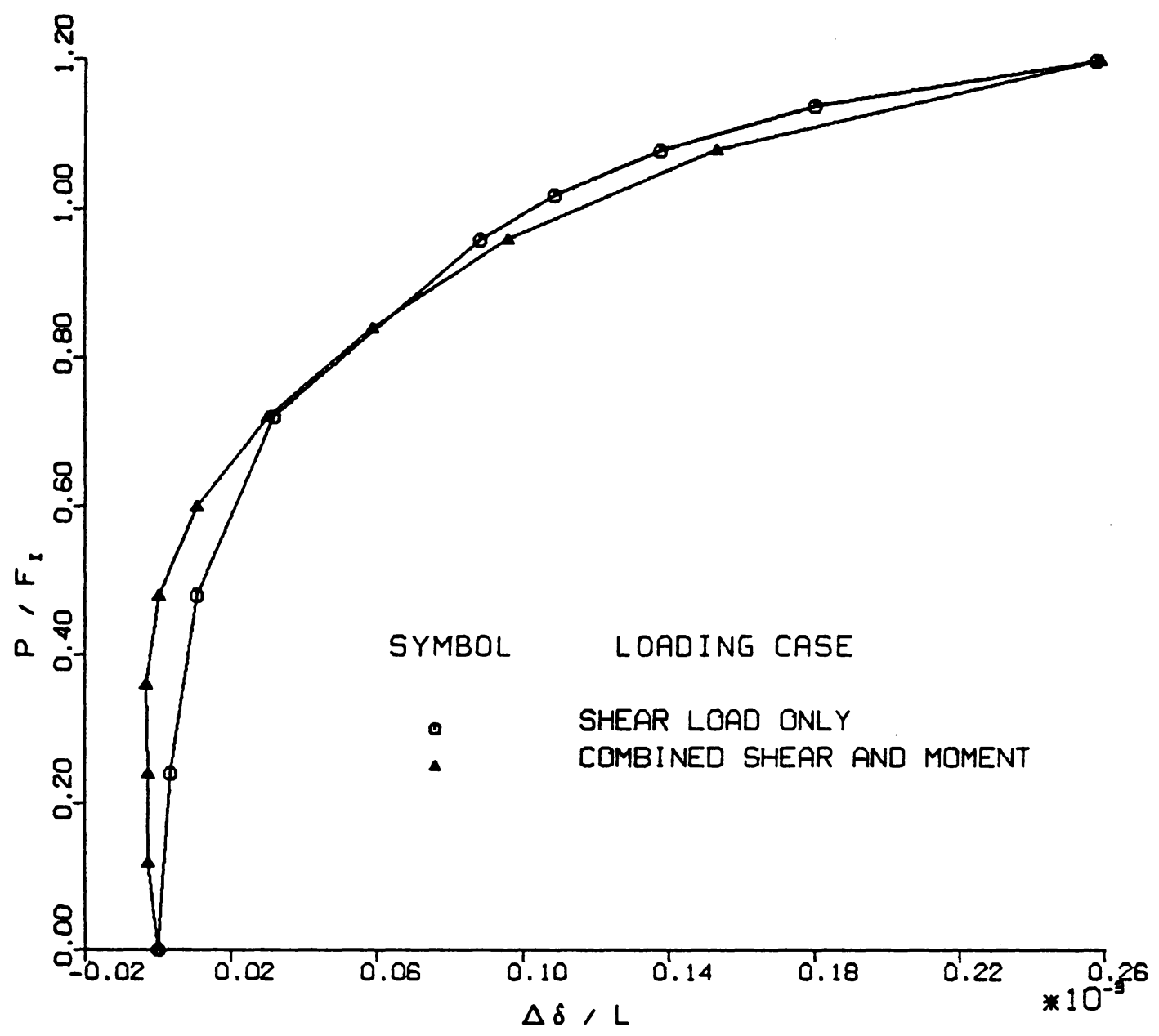

Figure 50. Applied Shear Load versus Bolt Strain for Combined Moment and Shear Loading $(\mu=0.1)$ 
This can be seen by the decrease in the bolt strain relative to preload in the first positive combined shear and moment load step. This agrees with the analysis of the previous section which revealed that bending action reduces the bolt strain relative to preload throughcompression relief. Figure 50 on page 97 also reveals that this bending action effect diminishes as the combined positive shear and moments increase and eventually work to increase bolt strains for a given applied shear load. This behavior was not observed in the moment-loading-only analysis.

The point of intersection of the combined and shear-only load case defines an applied shear load value, $P_{0}$. For applied shear loads less than $P_{0}$, the combined load-case analysis predicts smaller bolt strains than the shear-load-only load-case analysis. The opposite holds true for applied shear loads greater than $\mathrm{P}_{0}$. Thus, $\mathrm{P}_{0}$ represents the maximum applied positive shear load to the bolted joint model in which the bolt strain results for the shear-load-only case are conservative compared to bolt strains from the combined shear-and-moment load case. It can be seen from Figure 50 on page 97 that $\mathrm{P}_{0} \cong 0.86 \mathrm{~F}_{\mathrm{i}}$

\subsection{Sensitivity Studies for the Bolted-Joint Finite Element}

\section{Model}

Various analysis runs were made to assess the sensitivity of the results to certain model parameters. The effect of load step size, thread element material properties, interfacial coefficient of friction, and bolted-joint model size were studied. Results are presented in the following subsections. 


\subsubsection{The Effect of Load Step Size}

Since the bolted-joint model includes friction along the model interfaces, the system is nonconservative. This means that the results are load-path dependent. To assess this effect, two refined load step analyses were carried out. The results are shown in Figure 51 together with the standard eleven load step analysis results. The $0.08 \mathrm{~F}_{\mathrm{i}}$ load step increments between 0 and $0.24 \mathrm{~F}_{\mathrm{i}}$. applied loads reveal even higher joint stiffnesses, with essentially an infinite joint stiffness for the first $0.08 \mathrm{~F}_{\mathrm{i}}$ applied load from preload. The refined loading analysis between $0.96 \mathrm{~F}_{\mathrm{i}}$ and $1.2 \mathrm{~F}_{\mathrm{i}}$ indicates complete joint separation occurs between an applied tensile shear load of $1.08 \mathrm{~F}_{\mathrm{i}}$ and $1.14 \mathrm{~F}_{\mathrm{i}}$. Most importantly, though, the refined loading analysis reveals little path dependence of the standard eleven load step analysis.

\subsubsection{The Effect of the Thread Element Material Properties}

Since considerable effort was put forth to verify and implement Bretl's special "thread" elements in the bolted-joint finite element model, it was important to assess their effect on the results. A standard eleven load step analysis was made with normal isotropic material properties used for the thread elements instead of the orthotropic material properties. Figure 52 shows that the thread element material properties have a significant effect on the results. Applied tensile shear loads between 0 and $0.48 \mathrm{~F}_{\mathrm{i}}$ result in an actual decrease in the bolt strain from preload for the isotropic thread-element analysis. This corresponds to a negative joint stiffness effect. In terms of predicting the bolt strain as a function of the applied shear load, the orthotropic thread-element analysis is seen to be conservative compared to the isotropic thread-element analysis. That is, the 


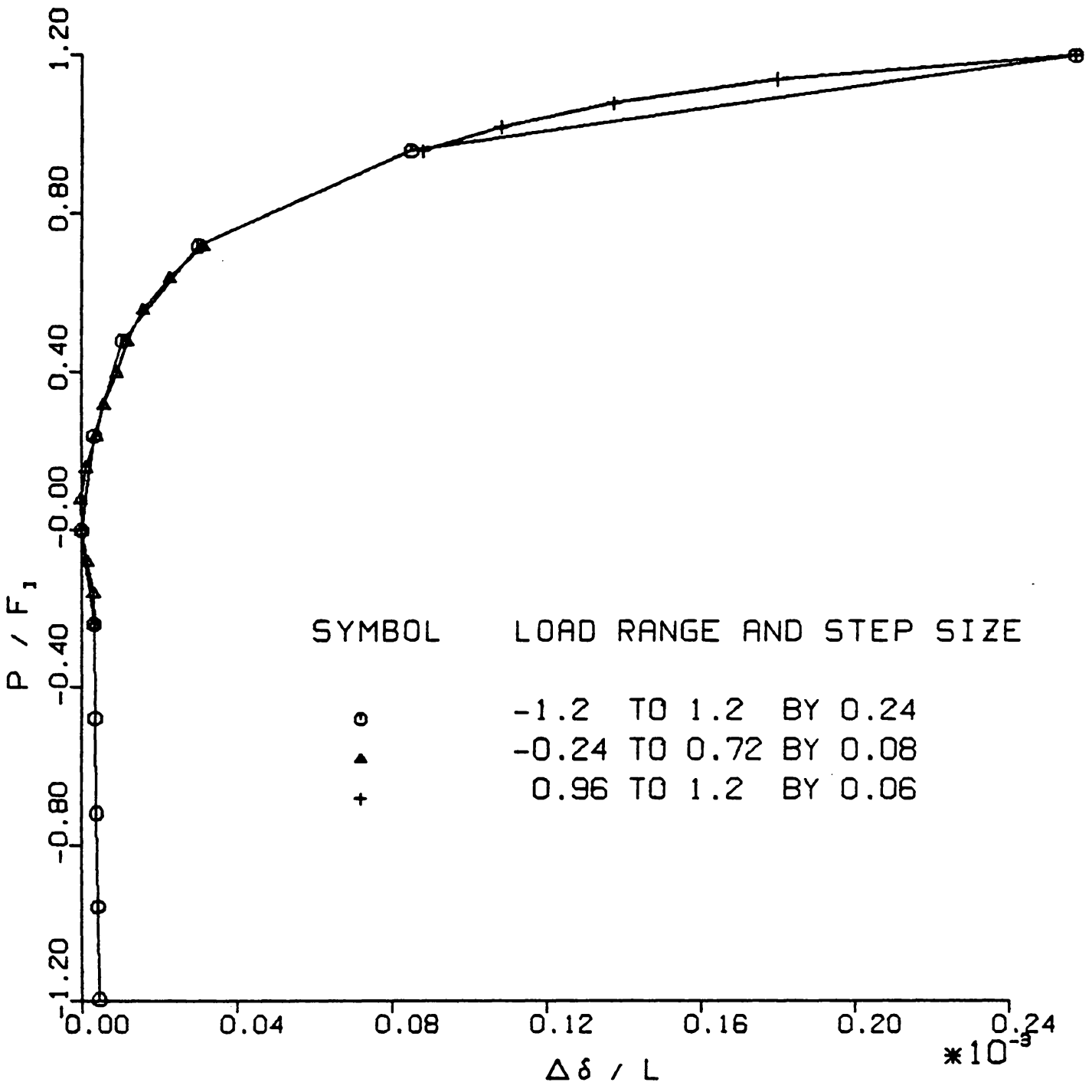

Figure 51. The Effect of Load Step Size: Applied Shear Load versus Bolt Strain $(\mu=0.1)$ 


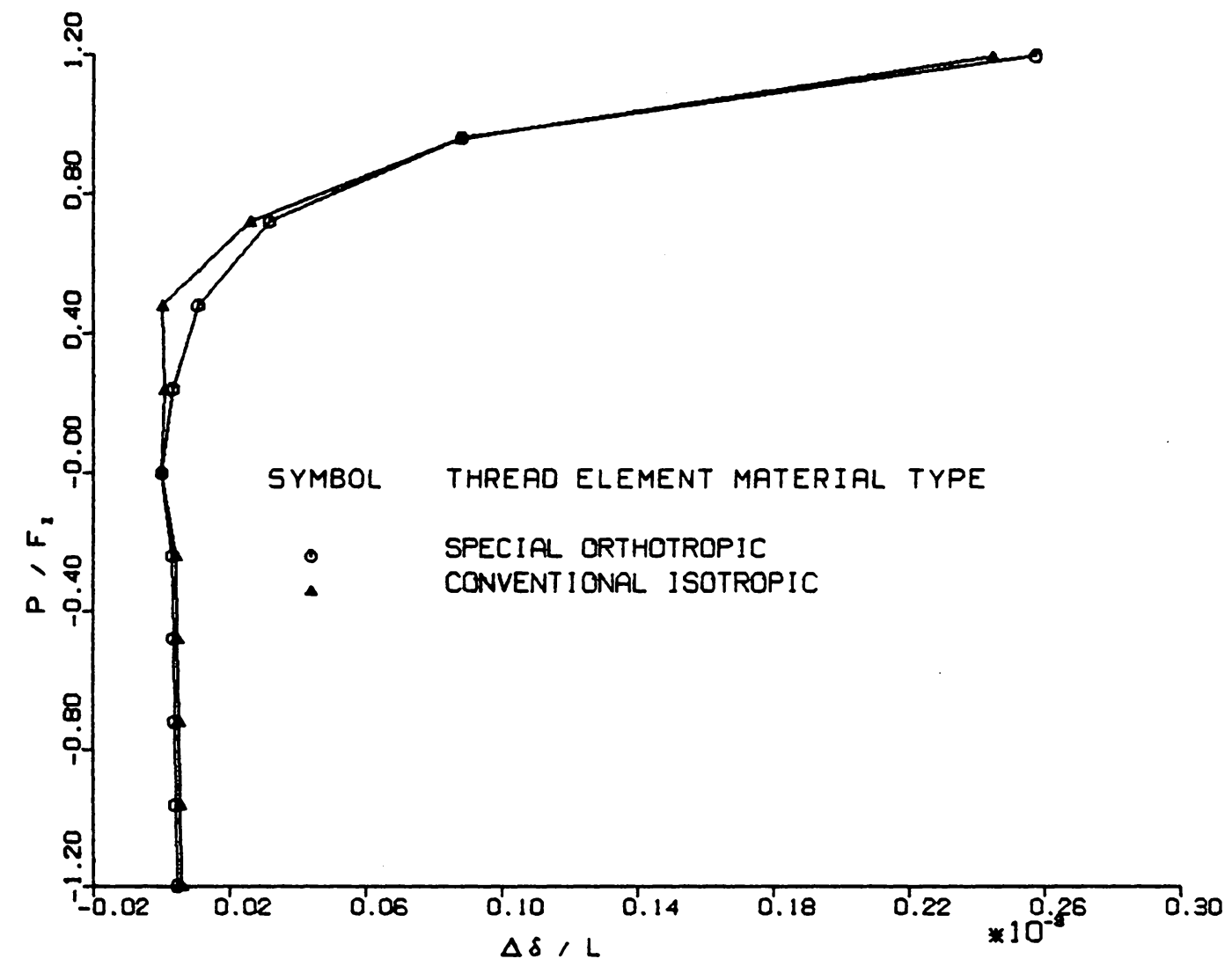

Figure 52. The Effect of the Thread Element Material Properties: Applied Shear Load versus Bolt Strain $(\mu=0.1)$ 
orthotropic thread-element analysis predicts larger bolt strains than the isotropic thread-element analysis. Therefore, it is safer to use orthotropic thread-element analysis results when estimating bolt loads than using the results of the isotropic thread-element analysis.

This author suggests that the differences between the results obtained using special orthotropic versus isotropic thread elements is related to rotation of the joint members. Specifically, it is imagined that the bottom joint member rotates about some point on the nut bearing surface when tensile shear loads are applied. Because the special orthotropic thread-elements permit a rather large nut rotation, this pivoting point on the nut bearing surface is suspected to exist approximately halfway between the inner and outer nut radius when the applied tensile shear loads are relatively small. Therefore, rotation of the bottom joint member under applied tensile shear loads reduces pressure forces inside the radius of the pivot point and increases pressure forces on this interface outside the radius of the pivot. Of course, superimposed on this action is axial translation of the joint member which seeks to increase the pressure forces across the entire bearing surface and hence the loads carried by the bolt. When the special orthotropic thread elements are replaced with conventional isotropic elements, there is significantly less nut rotation. Therefore, for this case, it is believed that the pivot point of the bottom joint member is located closer to the outer nut radius for small applied shear loads. Rotation of the joint member results in more pressure relief inside the pivot point radius because of the larger radius. The net effect is an increase in joint stiffness relative to the joint stiffness obtained using orthotropic thread elements. The fact that there is actually a decrease in the bolt strain relative to preload in the first two tensile load steps of the isotropic thread-element analysis indicates that compression relief due to joint member 
rotation is greater than any increase in tension due to axial translation of the joint members.

This concept of pivot points on the bolt head and nut bearing surface also helps explain why the results of Figure 52 on page 101 converge for larger tensile shear loads. As the tensile shear loads increase, the pivot points in both cases move radially inward toward the inside edge of the bolt head and nut. Eventually, there is little difference in the pivot point locations. Furthermore, as discussed earlier, the ability of joint member rotation to relieve compression decreases as the size of the compressed-member region decreases.

\subsubsection{The Effect of Interfacial Friction}

Figure 53 shows the effect of friction in the interfaces of the bolted-joint finite element model for the case of applied shear loading. The coefficient of friction, $\mu_{\text {thd }}$, used to calculate the inclination angle of the thread elements (see Figure 13 on page 37) was kept at its original 0.2 value for all analyses. Interfacial friction is seen to have a major effect for applied tensile shear loads and no effect for applied compressive shear loads. Increasing friction in the joint interfaces from 0.1 to 0.4 increases the bolt strains relative to preload for a given tensile loads between $0.24 \mathrm{~F}_{\mathrm{i}}$ and $1.2 \mathrm{~F}_{\mathrm{i}}$. Hence, the overall stiffness of the joint decreases with increasing interfacial friction. However, no further joint stiffness decrease is seen between 0.4 and 0.8 coefficient-of-friction results

Figure 54 shows the effect of interfacial friction together with the effect of combined shear and moment loading. The same comments made concerning the effect of interfacial friction for the shear load case can also be made for the combined shear and 


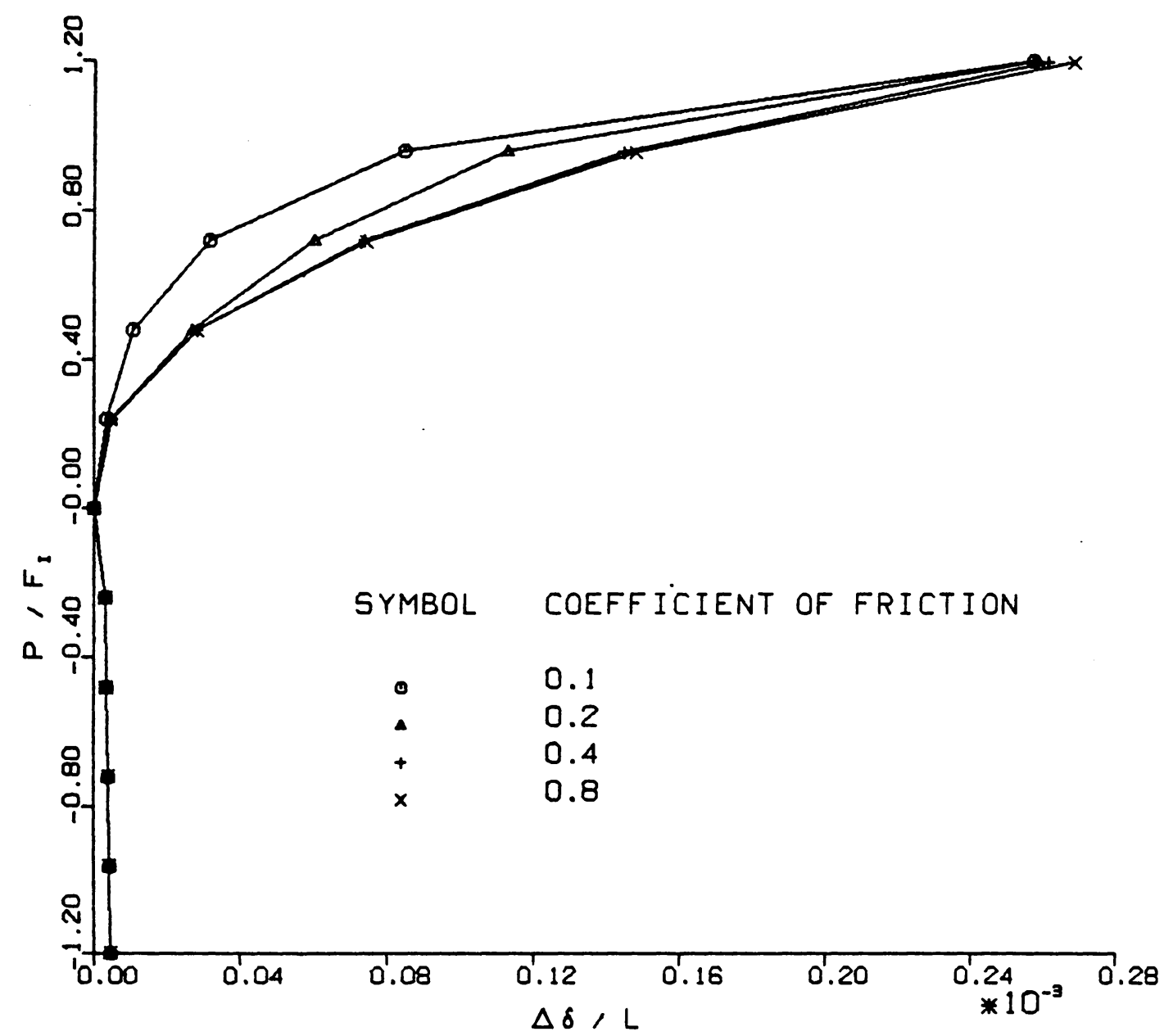

Figure 53. The Effect of Interfacial Friction: Applied Shear Load versus Bolt Strain 


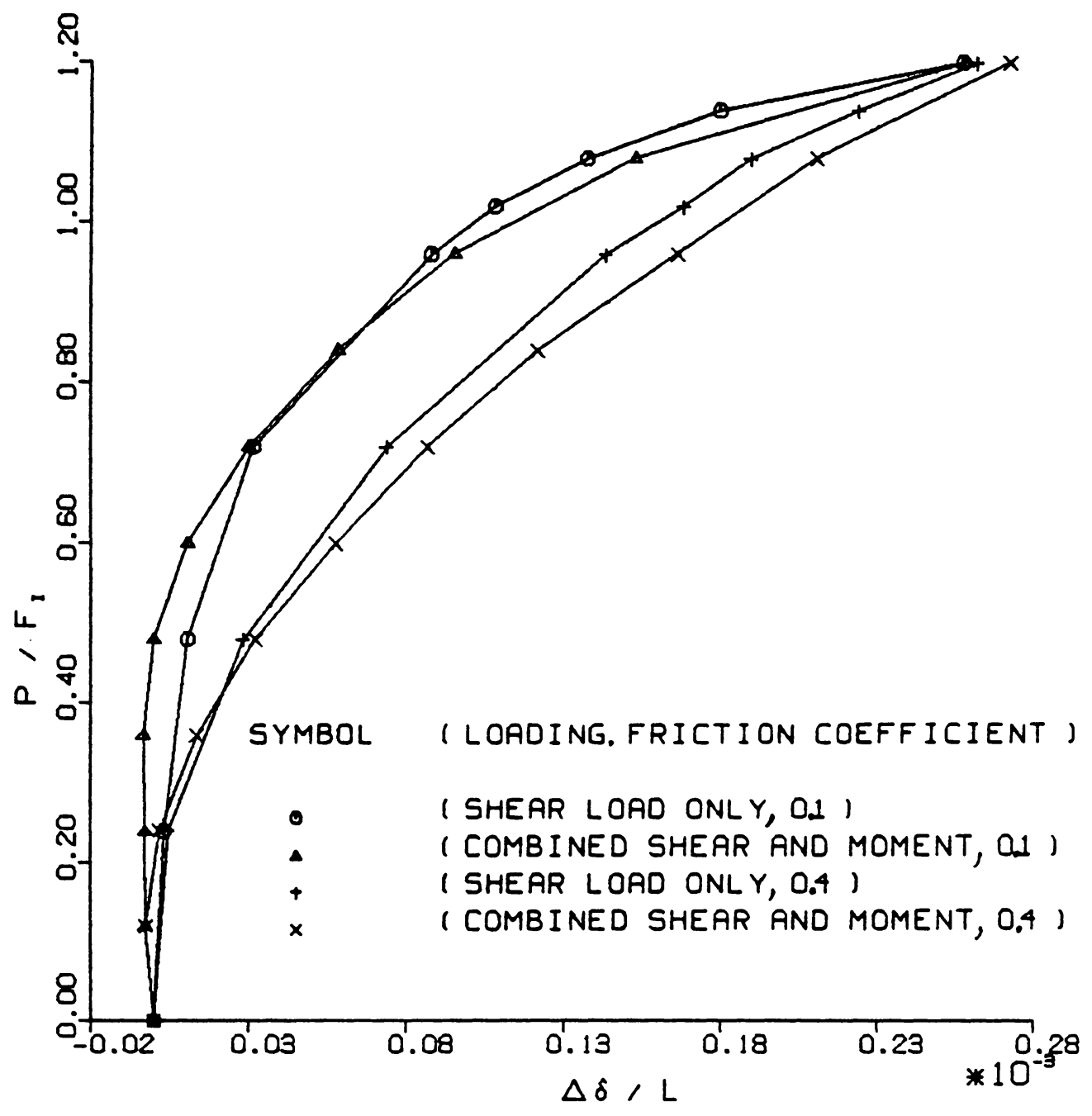

Figure 54. The Effect of Interfacial Friction: Applied Shear Load versus Bolt Strain 
moment load case. Note also that an increase in the friction coefficient from 0.1 to 0.4 results in a significant decrease in the load $\mathrm{P}_{0}$, the applied shear load defined by the intersection of the shear-only and combined loading curves. For a coefficient of friction of $0.4, P_{0} \cong 0.42 F_{i}$, whereas $P_{0} \cong 0.86 F_{i}$ for $\mu=0.1$. Therefore, increasing friction in the joint interfaces reduces the maximum applied positive shear load in which bolt strains of the shear-load-only analysis are conservative compared to the bolt strains of the combined loading analysis.

The same concept used to explain the different results obtained for the different threadelement material properties can explain the behavior seen in these friction studies. It is suspected that friction at the bolt head/plate interface and at the nut/plate interface plays a major role in determining the pivot points for rotations of the joint members. One can imagine that increasing interfacial friction hinders the slipping of the joint member on the nut surface thereby keeping the effective joint-member pivot point closer to the bolt axis. Increasing friction along these interfaces decreases the radius of the pivot points on the bolt head and nut bearing surface. Therefore, increasing friction reduces the ability of joint member rotation to provide compression relief on the bolt head/plate and nut/plate interfaces.

\subsubsection{The Effect of the Bolted-Joint Model Size}

The bolted-joint model of Figure 33 on page 72 has an outer edge radius $R_{o}$ equal to five times the thru-hole radius $r_{H}$. The effect of other model sizes between three times and five times the thru-hole radius is seen in Figure 55. The model size seems to have little influence on the bolt strains relative to preload for applied tensile shear loads. 


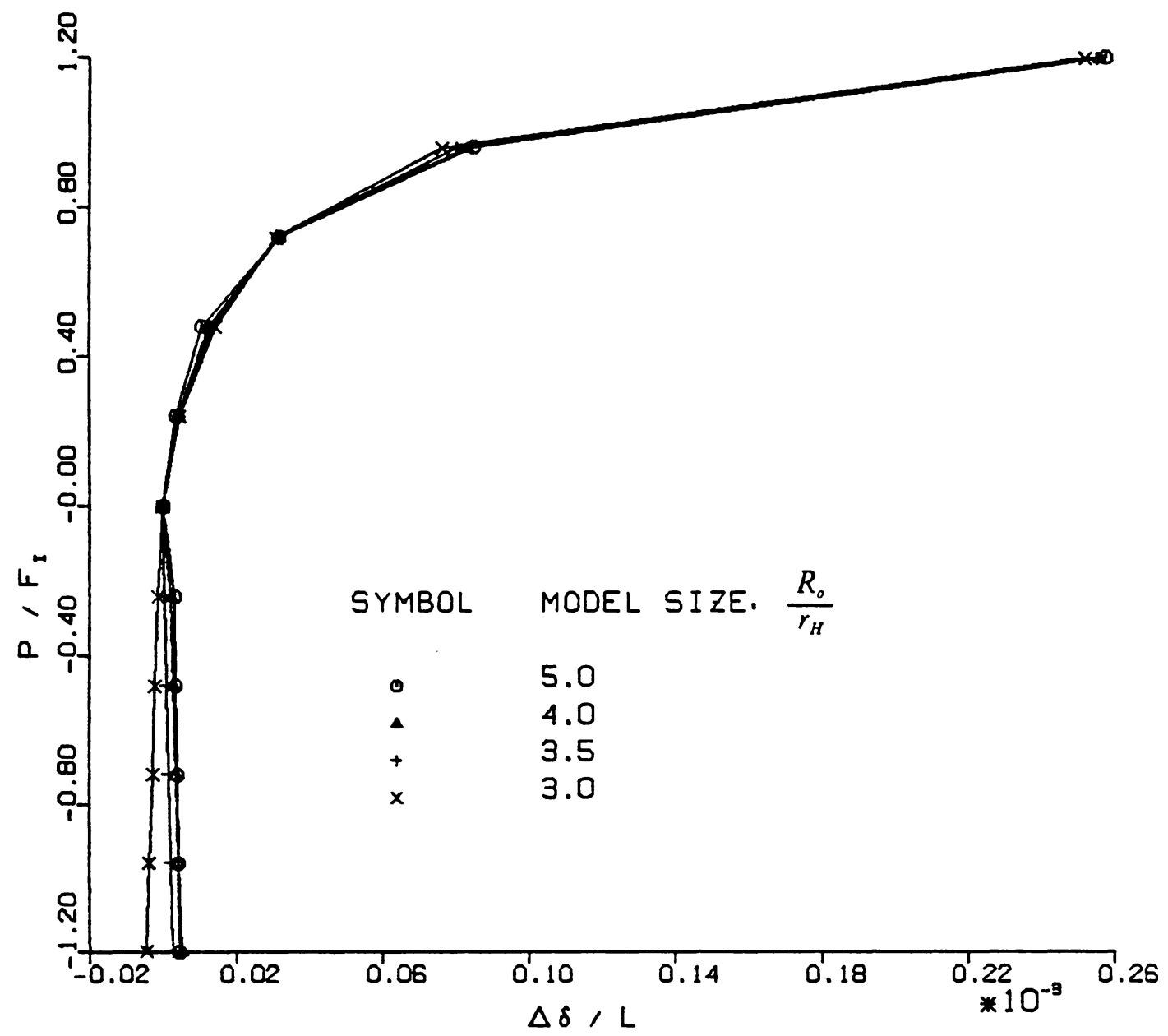

Figure 55. The Effect of the Bolted Joint Model Size: Applied Shear Load versus Bolt Strain $(\mu=0.1)$ 
A noticeable effect is seen for applied compressive shear loads. Increasing model size from 3.0 $r_{H}$ to $4.0 r_{H}$ causes increasing bolt strains for a given compressive shear load. The effect diminishes as the model size increases with no discernible effect seen between results for model sizes $=4.0$ and $5.0 r_{H}$. In terms of predicting the bolt strain, results of the selected bolted-joint model are conservative compared to the smaller model size.

This effect of model size is easily understood. As the model radius decreases, the effect of prying becomes less significant compared to the axial compression of the joint members due to the applied compressive shear loads. The axial joint member compression has the effect of relieving compression under the bolt head and nut and thereby decreasing the bolt strain relative to preload.

The limitations of these sensitivity studies should be realized. The nonlinearity of the bolted joint prevents superposition of results. Therefore, the results of sensitivity studies based on an individual parameter variation cannot be superimposed to obtain a correct description of the system under multiple parameter variation. Because of this lack of superposition, it is difficult to develop an intuitive understanding of complex nonlinear systems. This author has found this to be particularly true for the bolted-joint problem. 


\section{Chapter 6}

\section{Design Application of the Nonlinear Bolted-Joint}

\section{Theory}

Results of the previous chapter revealed the nonlinear stiffness of the bolted joint and many of the factors that influence the joint stiffness. The results will now be studied in terms of their impact on design. For this purpose the results are formatted in terms of a bolted-joint diagram. The preload bolt strain is first calculated by computing the preload axial bolt extension using Eq. (3.7) and dividing by the bolt length $L_{b}$ between the bolt head and nut. The bolt preload strain is then added to the bolt strains relative to preload to yield the total bolt strain. Figure 56 shows a nonlinear bolted-joint diagram generated using the finite element results based on an interfacial friction coefficient of 0.1 . The results of the refined load step analysis near the critical joint-separation load has also been included in the figure for greater accuracy. Now the magnitude of the bolt strains relative to preload can be compared to the magnitude of the bolt preload strain. In fact, the ratio of the bolt strain at joint separation to the bolt preload strain yields the 


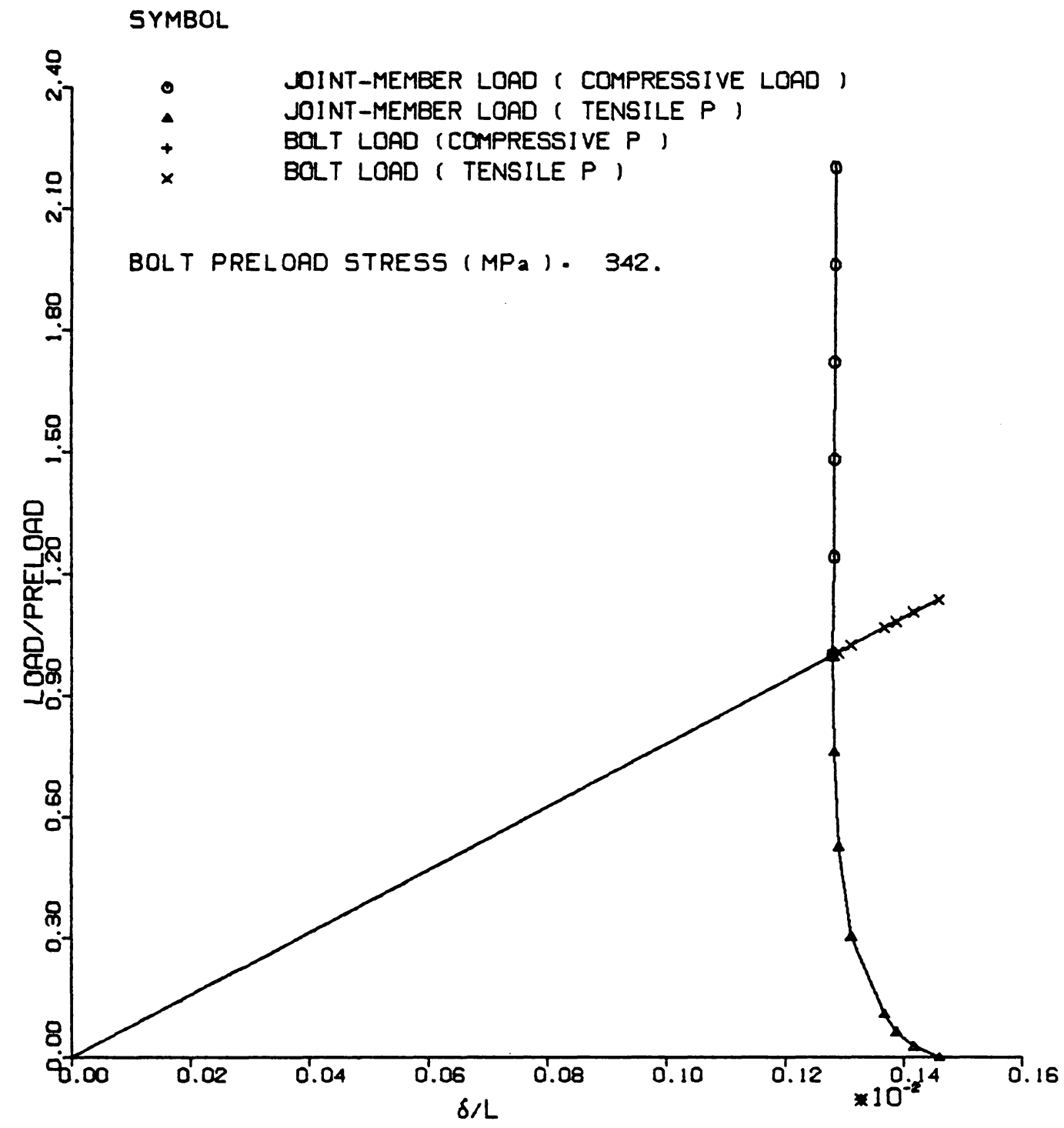

Figure 56. The Nonlinear Bolt Loading Diagram $(\mu=0.1)$ 
ratio of the critical joint-separation load $P_{c}$ to the bolt preload $F_{i}$. The joint member loads were computed by summing up the gap element forces along the plate/plate interface for each load step. The bolt loads were obtained by extracting and summing up the internal nodal forces on a horizontal plane through the bolt. The bolt load data points for the case of compressive applied loads are difficult to see because the additional loads are relatively small compared to the bolt preload. These data points are clustered on the bolt load line near the preload state. Note also that the joint member load curve has also been extended for the case of compressive applied shear loads.

To see the nonlinear, joint-member load curve in greater detail, the bolt preload strain has been removed in the bolt loading diagram of Figure 57. Also shown in Figure 57 is a linear joint-member load curve calculated from the linear theory using a double conical frustrum model with a 45 degree half-conical-face angle and intermediate loading plane factor of $1 / 2$. Recall from Chapter 2 that the bolt load is obtained by fitting the scaled load vector vertically between the bolt load and joint-member load curve. An example is shown in Figure 57 with a load vector equal to $40 \%$ of the preload. The finite element results predict an additional bolt load $P_{b} \cong 0.0057 F_{i}$, whereas the linear theory predicts $P_{b} \cong 0.045 F_{i}$. For this applied shear load, the linear theory yields an additional bolt load almost eight times greater than the finite-element-based additional bolt load. A compressive load vector of the same magnitude has also been fitted between the compressive joint-member load curve and the bolt load curve. The resulting additional tensile bolt load due to this applied compressive load is $0.0023 \mathrm{~F}_{\mathrm{i}}$. Although not shown in Figure 57, the linear theory handles applied compressive loads by linearly extending the linear joint-member load curve across the bolt load curve. For an applied compressive load of $0.4 \mathrm{~F}_{\mathrm{i}}$, the linear theory predicts $P_{b} \cong-0.045 F_{i}$, indicating a decrease in the bolt load. Because the bolt strains and additional bolt loads are relatively small 


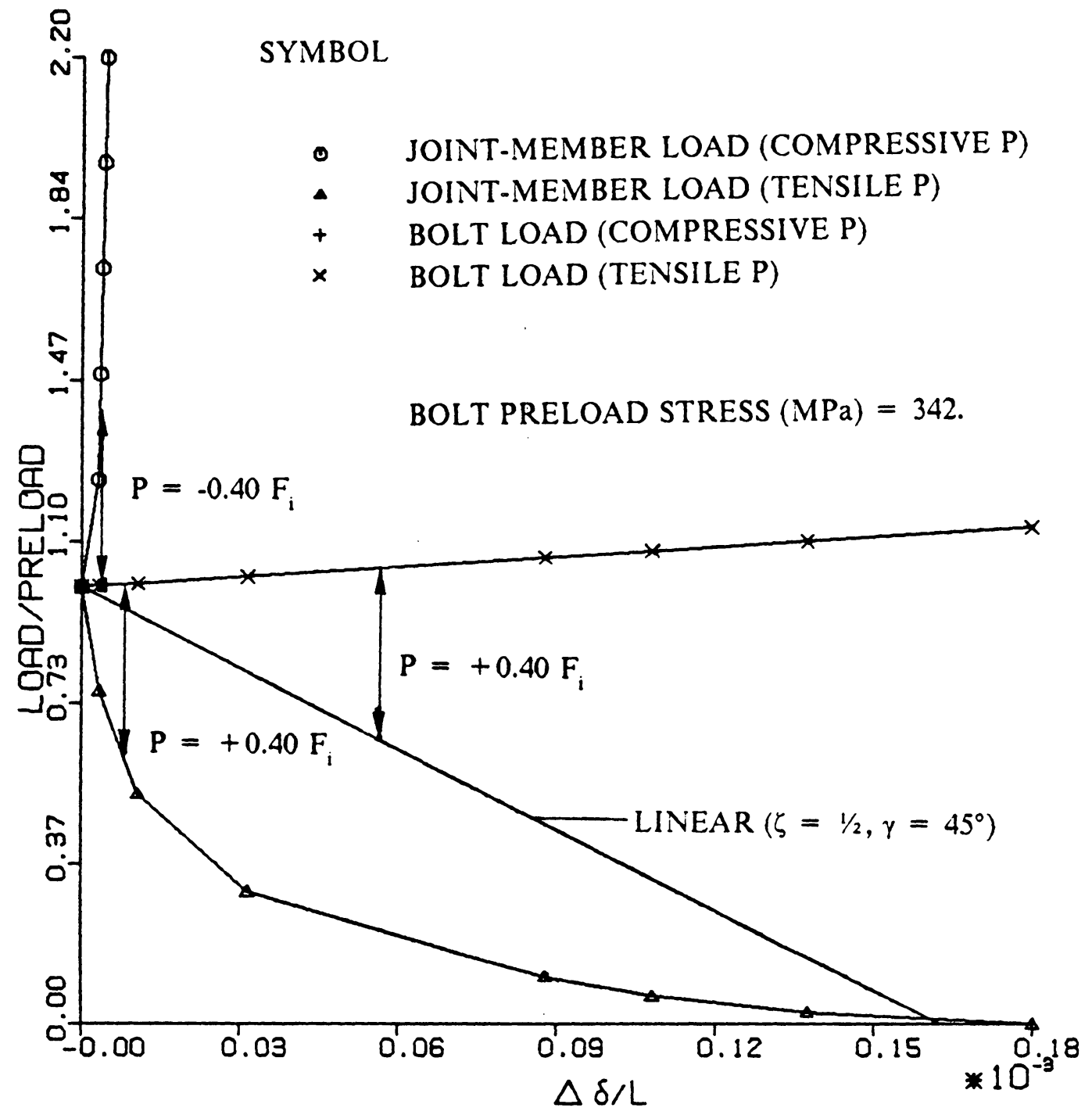

Figure 57. The Nonlinear Bolt Loading Diagram Relative to Preload $(\mu=0.1)$ 
for applied compressive loads, the portion of the joint-member load curve for applied compressive loads will not be included in subsequent bolt-loading diagrams. It is important to realize that applied compressive loads can increase the bolt load when one would intuitively expect a decrease from the classical concepts.

The nonlinear bolted-joint diagram presented here is similar to the diagram presented by Kwiatkowski et al. [27] shown in Figure 8 on page 23. The authors analyzed a high-strength bolted connection of similar proportions using elastic-plastic finite element analysis. The authors determined the effect of elastic-plastic behavior in the joint in terms of the nonlinear bolt-loading diagram. The high-strength joint was preloaded to $397 \mathrm{kN}$ yielding a bolt preload stress and bolt strain of $708 \mathrm{MPa}$ and $3.4\left(10^{-3}\right)$, respectively. These values are over twice the bolt preload stress and strain used here. The authors obtained critical joint-separation loads of $1.118 \mathrm{~F}_{\mathrm{i}}$ through $1.135 \mathrm{~F}_{\mathrm{i}}$ with experimental models. Their elastic and elastic-plastic finite element models yielded critical joint separation loads of $1.176 \mathrm{~F}_{\mathrm{i}}$ and $1.124 \mathrm{~F}_{\mathrm{i}}$, respectively. The critical joint-separation load of Figure 57 on page 112 is $1.123 \mathrm{~F}_{\mathrm{i}}$, which is in good agreement with Kwiatkowski et al. experimental data. This result gives a second experimentally based verification of the selection of the 0.1 interfacial friction coefficient.

For the purpose of presenting useful results to the design community, two functions have been fitted to the nonlinear joint-member load curve obtained from the finite element results. Figure 58 shows the data fits for the results based on an interfacial coefficient of friction 0.1 and applied shear loads only. The two-term exponential function was fitted using a least squares method [35] which incorporates all of the finite element data points into the solution. The exponential data fit has the form 


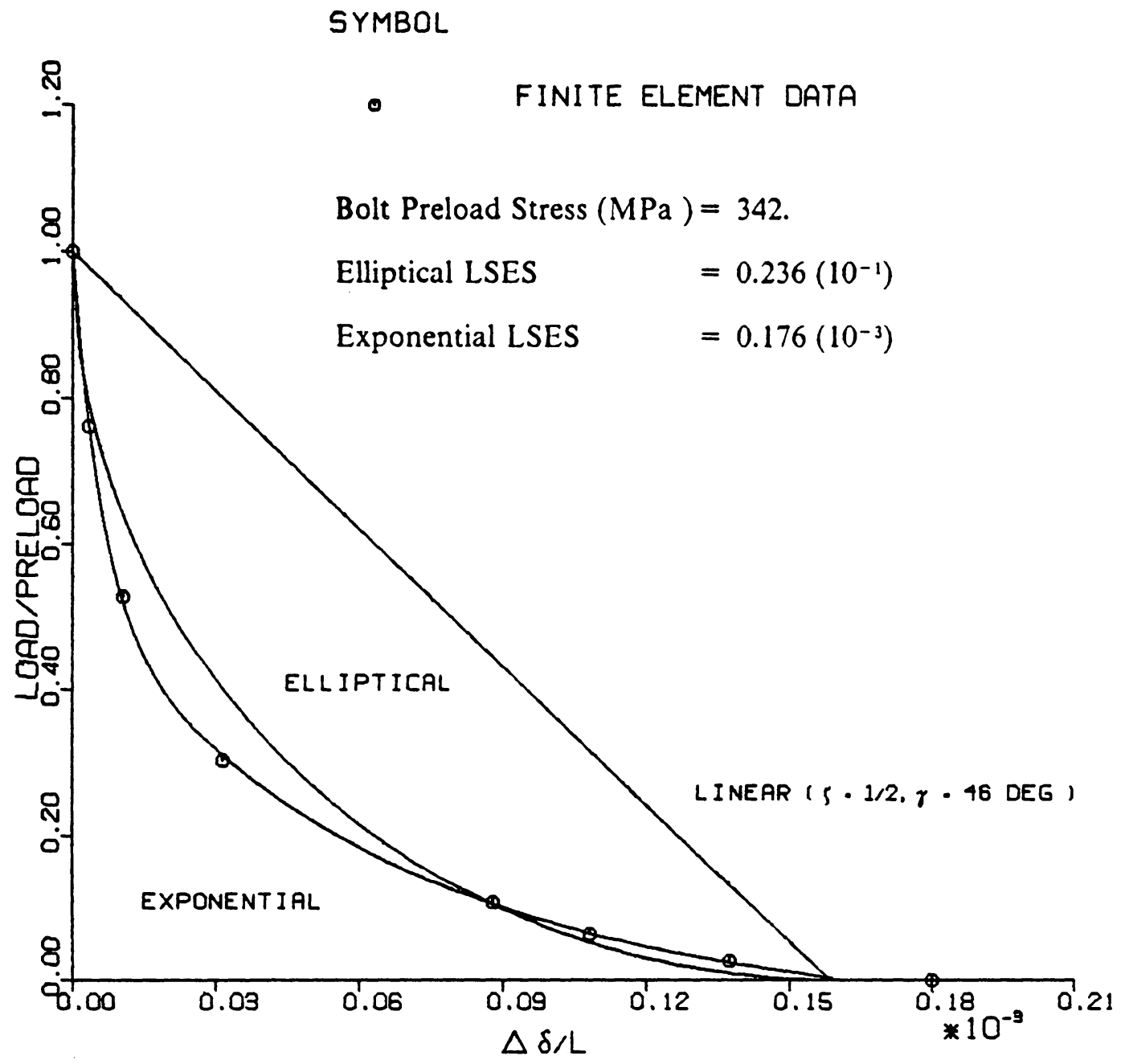

Figure 58. Data Fits for Joint-Member Load Data Curve $(\mu=0.1)$ 


$$
\bar{F}_{m} \equiv \frac{F_{m}}{F_{i}}=A+B \exp (C \Delta \varepsilon)+D \exp (E \Delta \varepsilon)
$$

where $\Delta \varepsilon$ is the bolt strain relative to the preload strain. The coefficients $A, B, C, D$, and E are obtained from the least-squares analysis.

The elliptical function shown in Figure 58 on page 114 is based only on the two finite element data end points corresponding to the preload state and the critical jointseparation state. The preload state is known apriori as $\bar{F}_{m}=1.0$ and $\Delta \varepsilon=0.0$. The critical joint-separation state is defined by the bolt strain at which the joint separates, termed the critical joint-separation strain $\Delta \varepsilon_{c}$, and zero load carried by the compressed member. The elliptical joint member load curve is given by

$$
\bar{F}_{m}=1.0-\left\{1-\frac{\left(\Delta \varepsilon-\Delta \varepsilon_{c}\right)^{2}}{\Delta \varepsilon_{c}^{2}}\right\}^{1 / 2}
$$

Therefore, only the critical joint-separation strain is needed to completely define the elliptical data fit as a function of $\Delta \varepsilon$.

The two-term exponential function provides a better fit to the finite element data but requires much more data to establish the curve. The residual Least-Squares Error Sum, LSES, is a measure of the deviation of the data values from the data fit

$$
\operatorname{LSES}=\sum_{i=1}^{n}\left(\bar{F}_{m}\left(\Delta \varepsilon^{i}\right)-\bar{F}_{m}^{i}\right)^{2}
$$

where $\mathrm{n}$ is the number of data points and $\left(\Delta \varepsilon^{i}, \bar{F}_{m}^{i}\right)$ is the $\mathrm{i}^{\text {th }}$ finite element data point of the nondimensionalized joint-member load curve. The residual Least-Squares Error Sums for the exponential and elliptical fits shown in Figure 58 on page 114 were com- 
puted to be $1.76\left(10^{-4}\right)$ and $2.36\left(10^{-2}\right)$, respectively. Compared to the exponential fit, the elliptical fit is conservative because it predicts larger bolt strains for applied tensile shear loads up to approximately the bolt preload.

Also shown in Figure 58 on page 114 is the linear joint-load curve which best fits the predicted critical joint-separation strain of the finite element analysis. This fit was obtained by setting $\zeta$, the intermediate loading plane factor, to $1 / 2$ and numerically solving the following nonlinear equation for the half-face-cone angle $\gamma$

$$
F_{i}-\frac{k_{m}(\gamma) L_{b} \Delta \varepsilon_{c}}{\zeta}=0
$$

where $k_{m}(\gamma)$ is a nonlinear function of $\gamma$ given by Eq. (2.7) and $L_{b}$ is the bolt length between the bolt head and nut.

If the designer is employing a joint configuration of similar proportions to the joint configuration considered herein, it is recommended that the two-term exponential function be used to determine the additional load carried by the bolt as a function of the expected loads entering the bolted-joint region. In general, though, the designer will be faced with a bolted connection of different proportions. For these cases, it is recommended that the designer use linear bolted-joint theory to estimate the critical jointseparation strain $\Delta \varepsilon_{c}$. Then use $\Delta \varepsilon_{c}$ to define the elliptical joint-member load curve. The additional bolt load can then be calculated using the elliptical joint-member load curve and the linear bolt-load curve for a given external load. This calculation will give more accurate additional bolt load values than using the simple, linear joint-member load curve. An outline of the procedure is given at the end of this chapter. 
Two appealing features of the elliptical fit are the slopes of the curve at the preload state and the critical joint-opening state. The finite element data indicates a near infinite slope of the joint-member load curve at the preload state. This means that virtually all of the applied axial loads in this region is absorbed by compression relief in the joint members. There is no additional load carried by the bolt. Hence, the one-dimensional jointmember stiffness is infinite at the preload state. On the other hand, one would expect a zero joint-member stiffness at the critical joint-separation load. This is because the joint-member stiffness is a measure of the ability of the joint members to absorb applied loads by compression relief. At the critical joint-separation state, all of the compression in the joint members has been relieved. Hence, all of the applied loads are carried by the bolt and the one-dimensional joint member stiffness is zero. The elliptical fit has the proper matching slopes at both the preload state and the critical joint-separation state.

Figures 59 through 61 compare the elliptical and exponential data fits of the jointmember load curve for $0.2,0.4$, and 0.8 interfacial friction coefficients. Refined load-step results for applied shear loads between $0.96 \mathrm{~F}_{\mathrm{i}}$ and $1.20 \mathrm{~F}_{\mathrm{i}}$ are included in the data fits to obtain more accurate critical joint-separation strains. These data fits are presented to show the effect of friction on the joint member load curve and offer the designer alternative joint-member load curves for analysis of bolted connections in which interfacial friction is known to be high. The residual Least-Squares Error Sums indicate excellent data fits for the two-term exponential functions. The best elliptical data fit was obtained in Figure 59 with a LSES value of $7.96\left(10^{-3}\right)$. Also shown in each figure is the linear joint-load curve which best fits the predicted critical joint-separation strain of the finite element analysis. 
SYMBOL

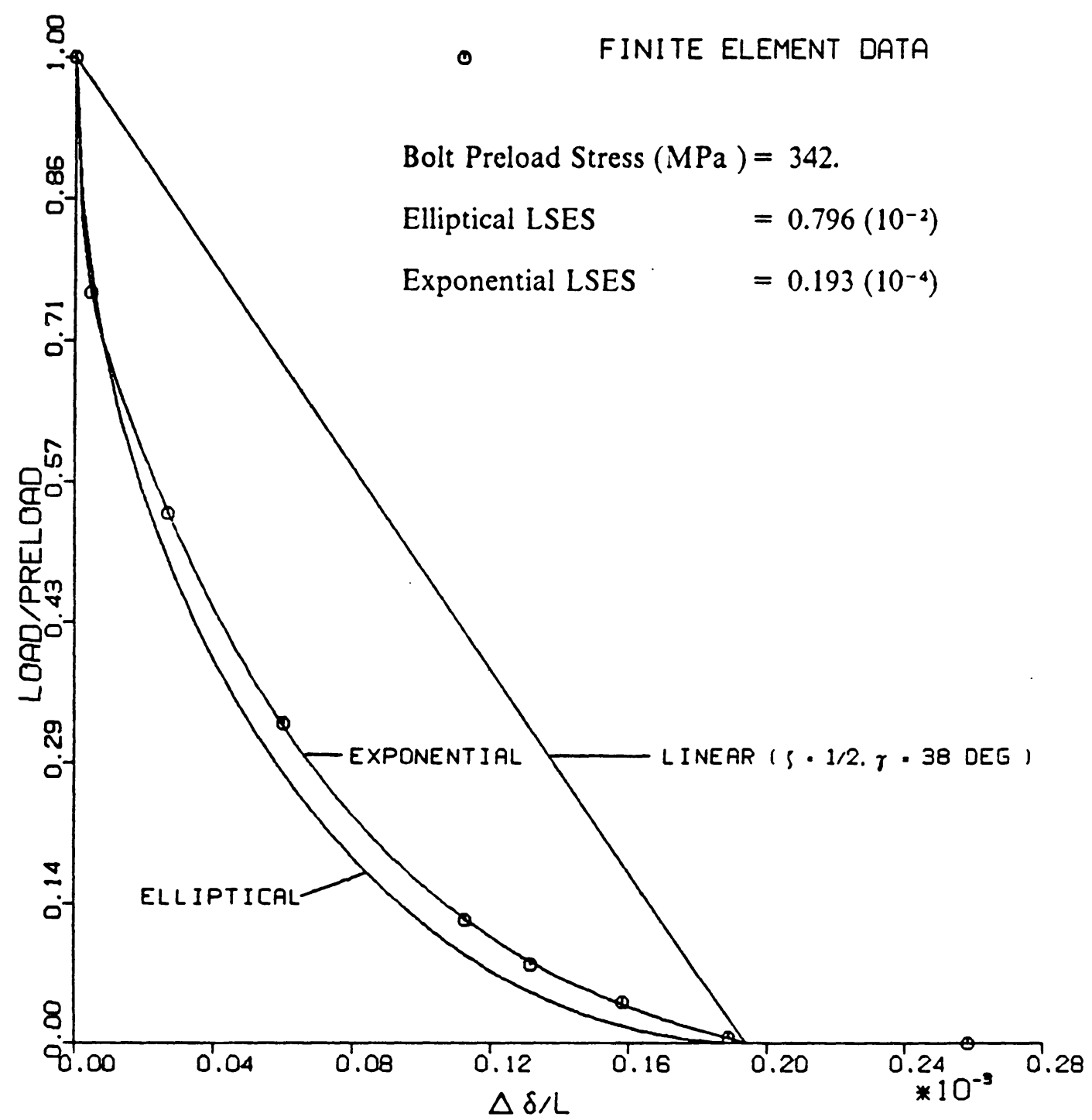

Figure 59. Data Fits for Joint-Member Load Data Curve $(\mu=0.2)$ 


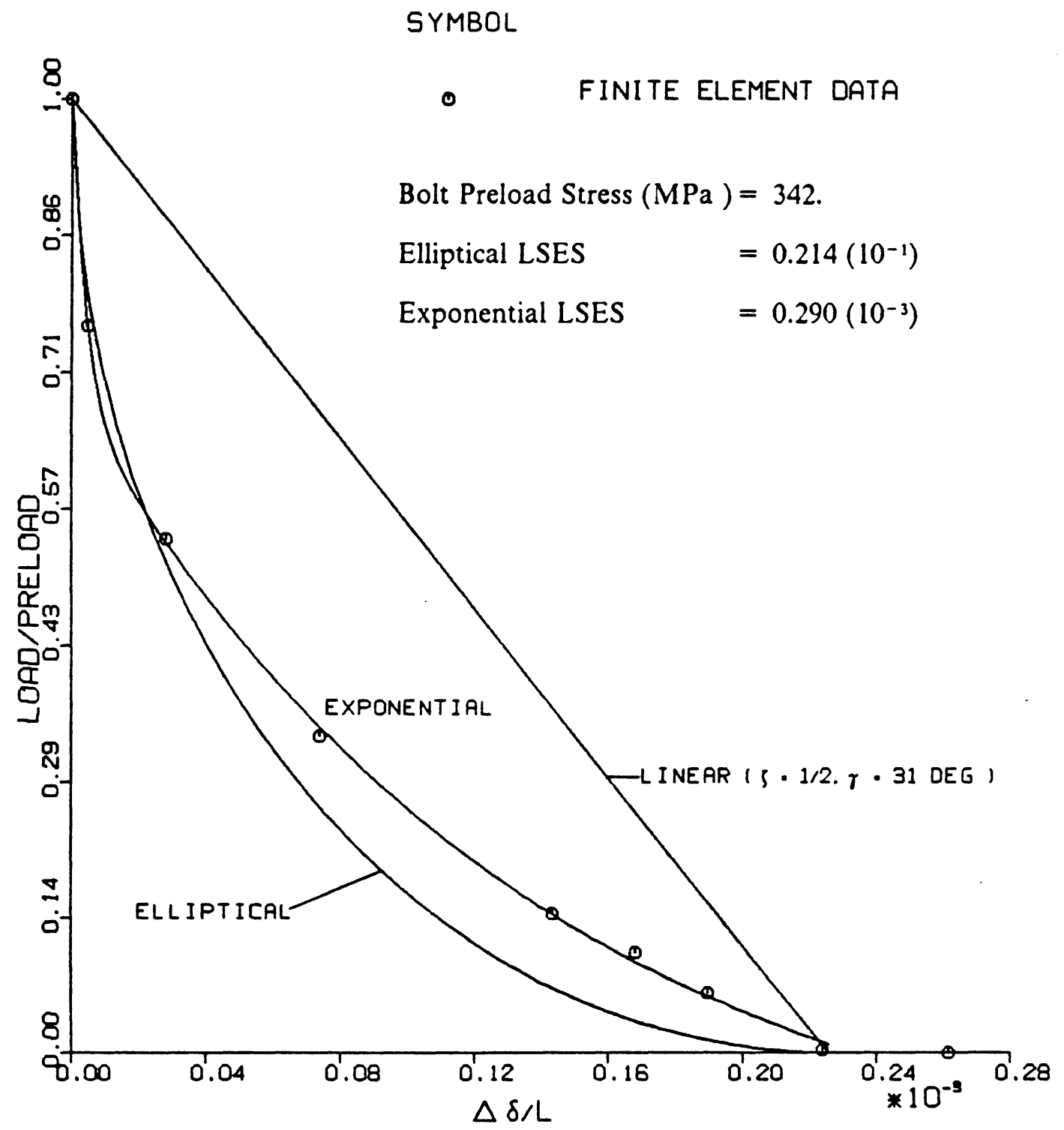

Figure 60. Data Fits for Joint-Member Load Data Curve $(\mu=0.4)$ 


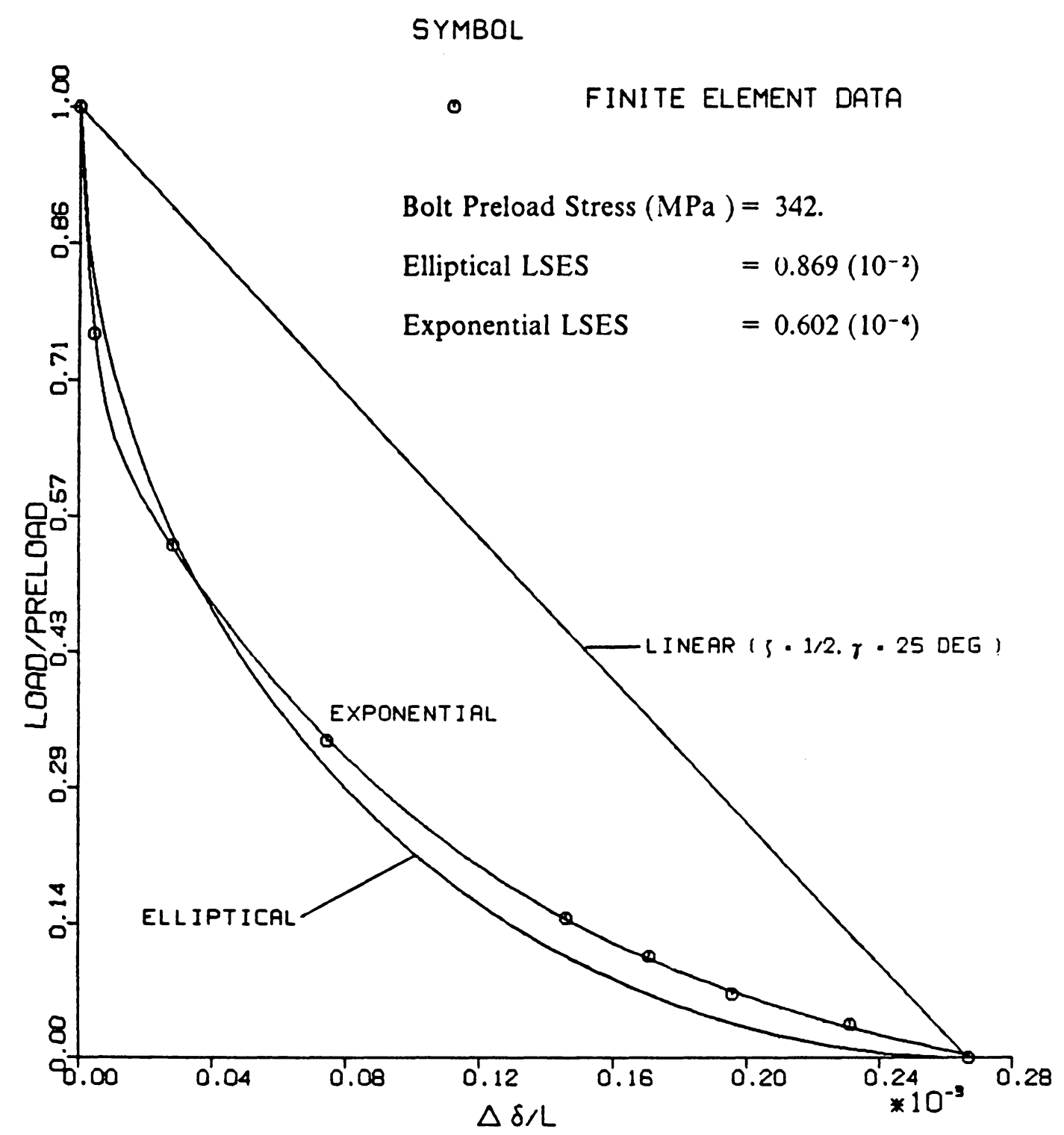

Figure 61. Data Fits for Joint-Member Load Data Curve $(\mu=0.8)$ 
Coefficients of the exponential function, the critical joint-separation strain (relative to preload), and the half-face-cone angle for the linear joint-member load curve are listed in Table 2 for each coefficient of friction. It can been seen from the exponential coefficients and Eq. (6.1) that each two-term exponential function includes a relatively large and small exponential decay coefficients. The large exponential decay coefficient reflects the extremely high joint-member stiffness near preload. The smaller decay coefficient reflects the joint-member stiffness away from the preload state.

When interpreting the finite element results for the various interfacial friction coefficients, one should keep in mind the work presented in Chapter 3 on adapting Bretl's special orthotropic thread elements into an ANSYS finite element model. A low interfacial coefficient of friction of 0.1 gave the best finite element results in terms of matching Goodier's empirical data on the radial nut expansion of his test specimen. Moreover, this was reconfirmed by the overall bolted-joint stiffness work of Kwiatkowski et al. [27]. The actual interfacial friction coefficient of the test specimen was not known. Hence, this friction coefficient value may be artificially low due to the inability of the special thread elements to simulate exactly the transfer of load between the bolt and nut. The insight gained from the results of Chapter 5 indicate that interfacial friction, especially at the nut/plate and bolt head/plate interface, may, indeed, have a significant effect on the nonlinear joint stiffness. This effect is seen in the results for the various interfacial friction coefficients.

Figure 62 shows the relationship between the exponential joint-member load curves in the bolt-loading diagram for the four interfacial friction coefficient analyses. The linear joint-member curve based on $\zeta=1 / 2$ and $\gamma=45^{\circ}$ is also shown in the figure. The intermediate loading plane factor of $1 / 2$ is recommended by Samonov [13] for the case of a 
Table 2. Data Fit Coefficients of Joint-Member Load Curves

\begin{tabular}{|c|c|c|c|c|c|c|c|}
\hline \multirow{2}{*}{$\begin{array}{c}\text { Coefficient } \\
\text { of Friction }\end{array}$} & \multicolumn{5}{|c|}{ Exponential Coefficients } & $\begin{array}{c}\text { Elliptical } \\
\text { Coeff. } \Delta \varepsilon_{c}\end{array}$ & $\begin{array}{c}\text { Angle } \\
\gamma(\text { deg })\end{array}$ \\
\hline \multirow{2}{*}{0.1} & -0.0530 & $\mathbf{B}$ & $\mathbf{C}$ & $\mathbf{D}$ & $\mathbf{E}$ & \\
0.2 & -0.0657 & 0.199 & $-1.64 \mathrm{E} 05$ & 0.572 & $-1.46 \mathrm{E} 04$ & $0.159 \mathrm{E}-03$ & 45.9 \\
0.4 & -0.171 & 0.311 & $-2.39 \mathrm{E} 05$ & 0.860 & $-6.93 \mathrm{E} 03$ & $0.226 \mathrm{E}-03$ & 31.3 \\
0.8 & -0.0779 & 0.294 & $-2.63 \mathrm{E} 05$ & 0.784 & $-8.50 \mathrm{E} 03$ & $0.267 \mathrm{E}-03$ & 24.5 \\
\hline
\end{tabular}




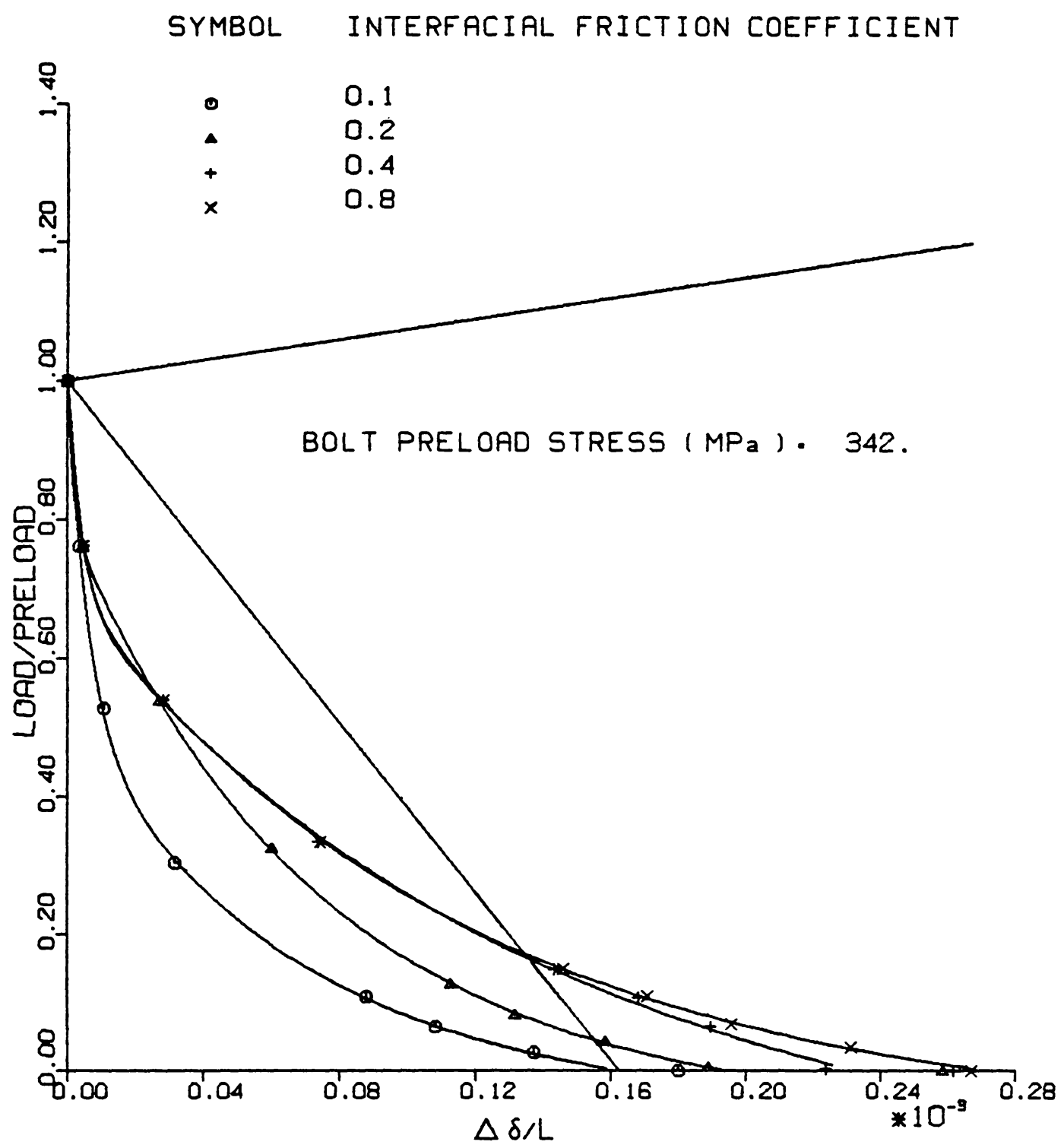

Figure 62. The Effect of Friction on the Nonlinear Bolt Loading Diagram 
uniformly distributed applied shear load. The $45^{\circ}$ half-face-cone angle of the double conical fructrum model is recommended by Shigley and Mitchell [7]. The figure shows that increasing friction decreases joint stiffness but increases the critical joint-separation load. More importantly, the linear theory is seen to be highly conservative in terms of predicting bolt strains. This can be seen more clearly in Table 3. Table 3 shows additional bolt loads calculated using the nonlinear exponential and linear joint-member load curves for various applied tensile shear loads. Included in parentheses are the ratios of $P_{b}$ obtained from the finite element results to $P_{b}$ obtained using linear theory. The linear theory is conservative for ratios less than 1.0 and nonconservative for ratios greater than 1.0. It should be noted that additional bolt loads corresponding to applied loads greater than the bolt preload are not included in Table 3, as well as additional bolt loads for the 0.8 interfacial friction coefficient case.

Note in particular from Table 3 how conservative the linear theory is in calculating additional bolt loads compared to the finite element results obtained using a 0.1 interfacial friction coefficient which gave good agreement with published data. For an applied axisymmetric shear load equal to $25 \%$ of the preload, the linear theory predicts an additional bolt load an order of magnitude larger than obtained through finite element analysis. Certainly, one should design conservatively. However, overestimating additional bolt loads by a factor of 10 can lead to costly and noncompetitive overdesigns of systems subject to fatigue loading. The low additional bolt loads shown in Table 3 explain the success in the growing practice of preloading bolts to $100 \%$ of their proof strengths for fatigue situations [32].

Since the above results are based on finite element analyses of the bolted joint with applied shear loads only, care must be taken if the bolted joint is under significant bending 
Table 3. Additional Bolt Loads for Various Applied Shear Loads

\begin{tabular}{|c|c|c|c|c|}
\hline & \multirow{2}{*}{\multicolumn{3}{|c|}{$\begin{array}{l}\text { Finite Element Model Results } \\
\qquad P_{b} / F_{i}(\mathrm{FEM} / \mathrm{LT}) \\
\text { Interfacial Coefficient of Friction }\end{array}$}} & \multirow{3}{*}{$\begin{array}{c}\begin{array}{c}\text { Linear } \\
\text { Theory }\end{array} \\
P_{b} / F_{i} \\
\zeta=0.5 \\
\gamma=45^{\circ}\end{array}$} \\
\hline & & & & \\
\hline$P / F_{i}$ & $\mu=0.1$ & $\mu=0.2$ & $\mu=0.4$ & \\
\hline 0.25 & $0.00282(0.100)$ & $0.00394(0.140)$ & $0.00388(0.138)$ & 0.0199 \\
\hline 0.50 & $0.00877(0.156)$ & $0.0277(0.494)$ & $0.0239(0.427)$ & 0.0561 \\
\hline 0.75 & $0.0289(0.343)$ & $0.0504(0.599)$ & $0.0642(0.762)$ & 0.0842 \\
\hline 1.0 & $0.0772(0.689)$ & $0.0976(0.871)$ & $0.121(1.08)$ & 0.112 \\
\hline
\end{tabular}


loads. The results of Chapter 5 indicate that as long as the bending loads and shear loads are not too large, then conservative additional bolt load estimates will be obtained using the finite element results based on applied shear loads only. There is a temptation to quantify how large these applied moments and shear loads need to be before the above results are no longer conservative. It was shown in the previous chapter that the shear-only finite element results are no longer conservative compared to combined shear-moment loading with applied shear loads greater than $0.86 F_{i}$ and $0.42 F_{i}$ for interfacial friction coefficients of 0.1 and 0.4 , respectively. However, the combined moment-shear load results are based on an assumed relationship between the shear load and moment as discussed in Chapter 3. It was necessary to make this assumption because it was impossible to consider every possible shear and moment load combination. Since the model is nonlinear, one cannot obtain a desired combined loading case by superimposing results of separate shear and moment load case analyses. It is believed that this assumed relationship between the applied bending moment and shear load was such that the applied moment for a given shear load represented a reasonable upper bound on the bending moment for most bolted-joint applications.

With these considerations in mind, it is recommended that either elliptical or exponential joint-member load curves be used in analyzing joints with low-to-normal friction coefficients. It is highly unlikely that combined shear and bending loads will be large enough to make the analysis nonconservative. For joints with high coefficients of friction and potentially high bending and shear loads, it is recommended that a linear joint-member load curve be used based on an intermediate loading plane factor of 0.5 and a double conical frustrum model with the appropriate half-face-cone angle obtained from Table 2. It should be noted that for most applications, the internal shear loads entering 
bolted-joint regions will be less than $50 \%$ of the bolt preload for bolted joints loaded to $90-100 \%$ of the bolt proof load.

\subsection{Outline For Calculating Additional Bolt Loads}

In this section a procedure is outlined which will enable the designer to estimate the additional bolt loads carried in a bolted joint subject to axisymmetric shear loads. Variables are defined in the Nomenclature section.

The axial bolt stiffness is calculated based on $E A / L$ stiffnesses for the various constant diameter sections of the bolt

$$
k_{b}=\frac{E}{\sum_{i=1}^{n} \frac{l_{i}}{A_{i}}}
$$

The bolt stiffness is nondimensionalized by the bolt head to nut length and by the bolt preload

$$
\bar{k}_{b}=L_{b} k_{b} / F_{i}
$$

The bolt-load curve (relative to preload) in nondimensional form is given by

$$
\bar{F}_{b}=\frac{F_{b}}{F_{i}}=1.0+\bar{P}_{b}=1.0+\bar{k}_{b} \Delta \varepsilon
$$

The difference between the bolt load and joint load equals the applied shear load P. Normalizing again by the preload yields 


$$
\bar{P} \equiv \frac{P}{F_{i}}=\bar{F}_{b}-\bar{F}_{m}
$$

Substituting Eq. (6.7) for $\bar{F}_{b}$

$$
\bar{P}=1.0+\bar{k}_{b} \Delta \varepsilon-\bar{F}_{m}
$$

The appropriate joint-member load function $\bar{F}_{m}$ is then inserted into Eq. (6.9) for $\bar{F}_{m}$. Equation (6.9) is solved for $\Delta \varepsilon$ which is used to calculate the additional bolt load by

$$
\bar{P}_{b} \equiv \frac{P_{b}}{F_{i}}=\bar{k}_{b} \Delta \varepsilon
$$

The two-term exponential data fit has the form

$$
\bar{F}_{m}=A+B \exp (C \Delta \varepsilon)+D \exp (E \Delta \varepsilon)
$$

The coefficients A,B,C,D, and E are obtained from a least-squares fit of the finite element results. See Table 2 for values obtained for the bolted joint studied here. Substitution of Eq. (6.11) into Eq. (6.9) yields a nonlinear equation in terms of $\Delta \varepsilon$ which cannot be solved explicitly for $\Delta \varepsilon$. A numerical procedure must be used to obtain the solution. Once $\Delta \varepsilon$ is obtained, Eq. (6.10) is used to estimate the normalized bolt load.

The elliptical load-member curve is given by

$$
\bar{F}_{m}=1.0-\left\{1-\frac{\left(\Delta \varepsilon-\Delta \varepsilon_{c}\right)^{2}}{\Delta \varepsilon_{c}^{2}}\right\}^{1 / 2}
$$

The critical joint-separation strain $\Delta \varepsilon_{c}$ is estimated using the linear joint-member load curve for intermediate loading planes 


$$
\Delta \varepsilon_{c}=\frac{1.0}{k_{m 2}}
$$

where $k_{m 2}=\frac{k_{m}}{\xi}$. Using Eq. (2.7) and an intermediate loading plane factor $\xi=1 / 2$, the compressed member joint stiffness for intermediate loading plane is

$$
k_{m 2}=\frac{k_{m}}{\xi}=\frac{\pi D_{H} E \tan \gamma}{\ln \left[\frac{\left(D_{N}+L_{T} \tan \gamma-D_{H}\right)}{\left(D_{N}+L_{T} \tan \gamma+D_{H}\right)} \frac{\left(D_{N}+D_{H}\right)}{\left(D_{N}-D_{H}\right)}\right]}
$$

Values of the half-face-cone angle $\gamma$ that yield critical joint-separation strains $\Delta \varepsilon_{c}$ via Eqs. (6.13) and (6.14) in agreement with the finite element results are listed in Table 2. Once $\Delta \varepsilon_{c}$ has been calculated, it is then substituted into Eq. (6.12) to completely determine the elliptical load member curve. Equation (6.12) can be simplified to

$$
\bar{F}_{m}=1.0-\sqrt{2 \lambda-\lambda^{2}}
$$

where $\lambda \equiv \frac{\Delta \varepsilon}{\Delta \varepsilon_{c}}$. Substituting Eq. (6.15) into Eq. (6.9) and rearranging yields

$$
\bar{P}-\bar{k}_{b} \Delta \varepsilon=\sqrt{2 \lambda-\lambda^{2}}
$$

Noting that $\Delta \varepsilon=\Delta \varepsilon_{c} \lambda$ and that $\bar{k}_{b} \Delta \varepsilon_{c}=\left(\bar{P}_{b}\right)_{c}$, the normalized additional bolt load at the critical joint-separation load $\bar{P}_{c}$, Eq. (6.16) becomes

$$
\bar{P}-\left(\bar{P}_{b}\right)_{c} \lambda=\sqrt{2 \lambda-\lambda^{2}}
$$

Squaring both sides, rearranging, and using the quadratic formula to solve for $\lambda$ yields

$$
\lambda=\frac{1.0+\left(\bar{P}_{b}\right)_{c} \bar{P} \pm \sqrt{1.0+2\left(\bar{P}_{b}\right)_{c} \bar{P}-\bar{P}^{2}}}{1.0+\left(\bar{P}_{b}\right)_{c}^{2}}
$$


The correct sign in front of the radical is the negative sign since $\lambda$ must be zero if $\bar{P}$ is zero. Using the fact that $\Delta \varepsilon=\Delta \varepsilon_{c} \lambda$, Eq. (6.18) is substituted into Eq. (6.10) to yield the following formula for the normalized additional bolt load.

$$
\bar{P}_{b}=\left(\frac{\left(\bar{P}_{b}\right)_{c}}{1.0+\left(\bar{P}_{b}\right)_{c}^{2}}\right)\left[1.0+\left(\bar{P}_{b}\right)_{c} \bar{P}-\sqrt{1.0+2\left(\bar{P}_{b}\right)_{c} \bar{P}-\bar{P}^{2}}\right]
$$

$\left(\bar{P}_{b}\right)_{c}$ can be related to the normalized critical joint-separation $\bar{P}_{c}$ by letting $\Delta \varepsilon=\Delta \varepsilon_{c}$ and $\bar{F}_{m}=0$ in Eq. (6.9).

$$
\begin{aligned}
\bar{P}_{c} & =1.0+\bar{k}_{b} \Delta \varepsilon_{c}=1.0+\left(\bar{P}_{b}\right)_{c} \\
\left(\bar{P}_{b}\right)_{c} & =\bar{P}_{c}-1.0
\end{aligned}
$$

Substituting Eq. (6.20) into Eq. (6.19) yields the normalized additional bolt load as a function of two parameters: the normalized applied load $\bar{P}$ and the normalized criticaljoint separation load $\bar{P}_{c}$.

$\bar{P}_{b}=\left(\frac{\left(\bar{P}_{c}-1.0\right)}{1.0+\left(\bar{P}_{c}-1.0\right)^{2}}\right)\left[1.0+\left(\bar{P}_{c}-1.0\right) \bar{P}-\sqrt{1.0+2\left(\bar{P}_{c}-1.0\right) \bar{P}-\bar{P}^{2}}\right]$

Using Eq. (6.21) one can immediately calculate the additional bolt load for a given applied tensile load if the joint preload and critical joint-separation loads are known. This makes bolted-joint analysis using the elliptical joint member load curve more practical because critical joint-separation loads are generally reported in the literature ([27]). If only the additional bolt load is needed, then it is not necessary to use the linear theory to calculate the critical joint-separation strain $\Delta \varepsilon$ via Eqs. (6.13) and Eq. (6.14) provided that $\bar{P}_{c}$ for the bolted connection can be obtained from existing data. 


\section{Chapter 7}

\section{Conclusions and Recommendations}

\subsection{Conclusions}

The finite element results presented here have shown the axial stiffness of a bolted joint to be highly nonlinear with applied axisymmetric loads. Results reveal that the joint exhibits an extremely high axial stiffness for relatively small applied tensile shear loads. As the applied tensile shear loads are increased, the joint stiffness decreases until the joint members have completely separated. At this point the joint stiffness is determined completely by the bolt stiffness which is constant. Recently, experimental and finite element work by Kwiatkowski et al. [27] have revealed similar axial joint stiffness characteristics. In contrast, the linear theory of bolted-joint analysis assumes a constant joint stiffness based on constant stiffness of compressed-member region. The linear theory does not take into account the change in size of the compressed-member region as tensile shear loads relieve joint compression. Furthermore, finite element stress analysis 
of the joint members under preload revealed an axial stress distribution radically different from the uniform axial stress distribution assumed in the linear theory to calculate the compressed-member stiffness.

Results indicate that the joint member stiffness is nonlinear due to compression relief which reduces the size of the contact region between the joint members. The rate at which compression is relieved as tensile shear loads are increased is governed by a complex interaction of joint member rotation, bending, and axial translation. It is believed that joint member rotation is mainly responsible for compression relief for small tensile shear loads. As the loads are increased, this effect is seen to diminish relative to the other factors.

The effect of bending in the joint members was assessed with simulated applied radial line moments on the outer joint model edge. Results indicate that bending can have a beneficial effect in terms of reducing bolt strain. This increased-joint-stiffness effect due to bending was seen for small to moderate applied positive moment/shear loads. However, bending was seen to have a detrimental effect on the joint stiffness for large combined positive moment/shear loads. That is, larger bolt strains (hence reduced joint stiffness) were observed for large combined moment/shear loads compared to results for large applied shear loads only.

The assumed size of the bolted-joint region modeled was seen to have no noticeable effect on the joint stiffness for the case of applied tensile shear loads and for the model sizes analyzed. The model size did have a strong influence on the joint stiffness for the case of applied compressive shear loads. A negative-joint stiffness effect was observed for the larger model sizes in which tensile bolt strains relative to preload occurred for applied compressive shear loads. Based on the small magnitudes of the bolt strain in- 
crements induced by compressive shear loads, it is concluded that compressive shear loading need only be accounted for in bolted joints loaded to yield and subjected to cyclic loading. Under these conditions, the small tensile bolt strains introduced by compressive shear loading could have an effect on the fatigue life of the bolt.

This so-called negative-joint-stiffness effect is not a violation of physical principles. The joint stiffness is calculated based on applied loads at the outer edge of the joint and corresponding bolt deformations. In the context of a matrix representation, this is a transfer flexibility. However, for the purpose of the simplified one-dimensional analyses of bolted joints, it is considered a stiffness term here.

The results indicate that interfacial friction has a significant effect on the joint stiffness. Increasing friction decreases the compressed member stiffness under an applied tensile shear load, i.e., the portion of external load carried by the bolt increases. Interfacial friction inhibits the sliding of the joint members relative to the bolt head and nut under applied tensile shear loads. Decreased sliding of the joint members is thought to reduce joint member rotation and thereby to reduce compression relief along the plate/plate interface.

The linear bolted-joint theory is seen to be extremely conservative for small to moderate applied tensile loads. Linear-theory bolt strains were estimated an order of magnitude higher and greater than those obtained through the finite element analyses for some load cases. This could result in costly overdesign of structures with a large number of bolted connections. In addition, the results indicate that the linear theory may be nonconservative for large applied tensile loads and relative large interfacial friction coefficient values. 
The results point to many deficiencies in the linear bolted-joint theory. First, the linear theory has no mechanism to account for the effect of friction on the joint stiffness. Second, one must assume an effective loading plane which has no physical basis in the bolted joint. Third, the uniform axial stress assumption is particularly weak, in view of the finite element results and a physical understanding of joint-member compression. Fourth, joint-member stiffness calculations based on this assumption are suspect. In practice, the joint-member stiffness calculation is usually "fudged" by altering the halfface-cone angle of the double frustrum model until agreement with experimental jointmember stiffness is obtained, if known.

For the purpose of providing design-orientated results to the engineering community, the finite element results were formatted into nonlinear bolt loading diagrams. Two nonlinear functions were fitted to the nonlinear joint-member load curves. The two-term exponential functions provided excellent fits to the finite element data but have the disadvantage of requiring joint-member load data before they can be constructed. The designer would have to obtain this joint-member load data through similar finite element analysis and/or experimentation. If the designer is faced with bolted connection of similar proportions as the bolted joint studied here, then one of the exponential functions presented in Chapter 6 could be employed.

A second, more practical, joint-member load function is proposed which provides a reasonable fit to the finite element results. This function is an elliptical function which requires only one parameter related to the critical joint-separation state to be determined.

An analytical procedure was offered which outlined the use of both of these nonlinear joint-member load functions for calculating additional bolt loads for given applied tensile 
shear loads. The use of the exponential data fit yielded a nonlinear equation which must be solved numerically. Using the elliptical joint-member load curve, a closed-form solution for the additional bolt load was obtained. The additional bolt load was found to be a function of two parameters only: the critical joint-separation load and the applied shear load. This result provides another reason for using the elliptical joint member load curve because critical joint-separation loads are generally reported in the bolted-joint literature (e.q. Kwiatkowski [27]), or they can be estimating using a linear model. The bolt-load and joint-stiffness calculations involving the proposed nonlinear load member functions, especially for the elliptical load member functions, are not complicated. When the parameters are nondimensionalized, the resulting equations are easily manipulated.

The finite element results were obtained for the case of axisymmetric loading conditions. This does impose limitations on the applicability of the results. However, as pointed out in Chapter 1, there are many engineering situations in which the joint loading can be approximated as axisymmetric. Applications where prying action is not expected to be significant is one example. Loads transmitted to individual bolt regions in tensile joint using a multiple-bolt pattern is another.

\subsection{Recommendations}

In view of the shortcomings of the linear theory, the finite element results obtained, and the limitations of the results, the following recommendations are made. 


\subsubsection{Recommendations for the Designer}

- For joints of similar proportions as studied here and not subject to prying action, a two-term exponential function should be used for the joint member load curve in the analysis and design of bolted joints. Coefficients for this function are presented in Table 2 in Chapter 6.

- For other joint configurations not subject to prying, the designer should use an elliptical joint member load curve. The critical joint-separation load which defines the elliptical function should be obtained from experimental data. If not available, then the linear theory can be employed to estimate the critical joint-separation load.

- For critical joints subject to cyclic tensile and compressive applied loads less than approximately $40 \%$ of the preload, the designer must allow for the possibility that applied compressive loads may result in increased bolt loads instead of reduced bolt loads as predicted by the linear theory. This can be accounted for by doubling the frequency of the cyclic loading and then considering only the alternating tensile load in the fatigue analysis.

\subsubsection{Recommendations for Future Research}

- Finite element analyses investigating the formulation of new thread elements based on more physical principles are recommended.

- Additional finite element analyses and experimentation studying the axial stiffness characteristics of other ANSI standard joint designs are needed.

- It is recommended that an experimental study be conducted to obtain data on the effect of interfacial friction. The interfacial friction coefficient could be varied 
through lubrication, cleaning, lapping, application of adhesives, etc., of the member/bolt head and the member/nut surfaces. The axial stiffness characteristics of the joint should be with the interfacial friction values if they can be accurately measured.

- The effect of other parameters on the axial joint stiffness needs to be studied, such as unequal plate thickness, various ratios of plate thickness to bolt hole radius, and the use of various washers.

- New joint designs need to be analyzed in an effort to increase joint stiffness. One possible joint design would be using plates with slight countersunk bolt thru holes on the plate/plate interfacial surfaces. This would force the compression region in the plates further out, thereby allowing greater compression relief through plate rotation. In addition, compressive reaction forces near the bolt hole at the plate/plate interface due to rotations of the plates would be eliminated which would also increase compression relief.

- A full three-dimensional, nonlinear finite element analysis needs to be performed to study the effect of nonaxisymmetric loading conditions on the axial joint stiffness. To improve the computational efficiency and reduce costs of such analyses, it is recommended that extensive substructuring of the linear portions of the model be used.

- Finally, a tremendous boon to the finite element analyses of structures using bolted connections would be the development of a bolted-joint finite element. An approach needs to be established on how such an element could be formulated using the joint stiffness characteristics obtained through detailed finite element analyses and experimentation. Initially, it is recommended that a simple, two-noded, two degree-of-freedom be formulated based on only the one-dimensional axial stiffness characteristics of bolted-joints. Once more bolted-joint data becomes available, 
such as the bending stiffness characteristics, the element could be expanded to include these effects. 


\section{References}

1. Talbot, T. F., "Bolt Failure in an Overhead Hoist," ASME Paper No. 83-WA/DE-20, 1983 Winter Annual Meeting, ASME, New York, NY.

2. Cook, R. D., Concepts and Applications of Finite Element Analysis, 2nd Edition, John Wiley \& Sons, New York, 1981.

3. Reddy, J. N., An Introduction to the Finite Element Method, McGraw-Hill, New York, 1984.

4. Segerlind, L. J., Applied Finite Element Analysis, John Wiley \& Sons, New York, 1976.

5. Bathe, K. J. and Wilson, E. L., Numerical Methods in Finite Element Analysis, Prentice-Hall, Englewood Cliffs, N. J., 1976.

6. Zienkiewicz, O. C., The Finite Element Method, 3rd Edition, McGraw-Hill, London, 1977.

7. Shigley, J. E., and Mitchell, L. D., Mechanical Engineering Design, 4th Edition, McGraw-Hill, New York, 1983.

8. Bickford, J. H., An Intoduction to the Design and Behavior of Bolted Joints, Marcel Dekker, New York, 1981.

9. Sharp, G. R., "Determination of Stiffness and Loading in Bolted Joints Having Circular Geometry," Bettis Atomic Power Laboratory, Westinghouse Electric Corporation, West Mifflin, PA, November 1975, NTIS No. WAPD-TM-1105, NTIS, Spreyfield, VA.

10. Rotscher, F., Die Maschinenelemente, Berlin, Springer, 1927. 
11. Birger, I. A., "Determining the Yield of Clamped Components in Threaded Connections," Russian Engineering Journal, Vol. 41, No. 5, 1961, pp. 35-38.

12. Ito, Y., Toyoda, J., and Nagata, S., "Interface Pressure Distribution in a Bolted-Flange Assembly," Journal of Mechanical Design: Trans. of ASME, Vol. 101, No. 2, April 1979, pp. 330-337.

13. Samonov, C., "Stiffness Constants in Bolted Connections: Their Determination and Influence on Resulting Working Loads," Mechanical and Chemical Engineering Transactions, Vol. MC2, No. 2, November 1966.

14. Motosh, I. N., "Determination of Joint Stiffness in Bolted Connections," Journal of Engineering for Industry: Trans. of the ASME, Vol. 98, Series B, No. 3, August 1976, pp. 858-861.

15. Fritsche, G., Grundlagen Einer Genaueren Berechnung Statisch und Dynamisch Beanspruchter Schraubenverbindungen, Dissertation, Berlin Technical University, 1966.

16. Radzimovsky, E. I., "Bolt Design for Repeated Loading," Machine Design, Vol. 24, No. 11, November, 1952, pp. 135-146.

17. Phelan, R. M., Fundamentals of Mechanical Design, Second Edition, McGraw-Hill, 1962, pp. 152-155.

18. Faires, V. M., Design of Machine Elements, Third Edition, The Macmillan Company, New York, 1965, pp. 164-173.

19. Meyer, G., and Strelow, D., "Simple Diagrams Aid in Analyzing Forces in Bolted Joints", Assembly Engineering, Vol. 15, No. 1, January 1972, pp. 5-33.

20. Gould, H. H., and Mikic, B. B., "Areas of Contact and Pressure Distribution in Bolted Joints," Journal of Engineering for Industry: Trans. of the ASME, Vol. 94, Series B, No. 3, August 1972, pp. 864-870.

21. Maruyama, K., Yoshimoto, I., and Nakano, Y., "On Spring Constant of Connected Parts in Bolted Joints," Bulletin of the JSME, Vol. 18, No. 126, December 1975, pp. 1472-1480.

22. Ziada, H. H., and Abd El Latif, A. K., "Loading Conditions in Bolted and Riveted Joints Affected by Plate Thickness Ratio," Journal of Mechanical Design: Trans. of ASME, Vol. 102, No. 4, 1980, pp. 851-857.

23. Ziada, H. H., and Abd El Latif, A. K., "Load, Pressure Distribution and Contact Area in Bolted Joints," Journal of the Institution of Engineers (India), Part $M E$, Vol. 61, November 1980, pp. 93-100.

24. Ziada, H. H., and Abd El Latif, A. K., "Determination of Bolted Joint Stiffness From Load and Deformation Analysis," ASME Paper No. 82-DE-W-3, ASME, New York, NY. 
25. Bretl, J. L., Finite Element Analysis for General Solids and Threaded Connections, Dissertation, University of Wisconsin-Madison, 1978.

26. Chapman, I., Newnham, J., and Wallace, P., "The Tightening of Bolts to Yield and Their Performance Under Load," Journal of Vibration, Acoustics, Stress, and Reliability in Design, Vol. 108, April 1986, pp. 213-221.

27. Kwiatkowski, J. K., Winnicki, L. A., and Krzyspiak, A., "Stress Analysis of Bolted Tensile End Plate Connections," Rozprawy Inzynierskie Engineering Trans., Vol. 34, No. 1-2, 1986, pp. 113-137 (in English).

28. American National Standards, Metric Hex Nuts, Style 1: ANSI B18.2.4.1M 1979, The American Society of Mechanical Engineers, New York, 1979, p. 4.

29. American National Standards, Metric Hex Bolts: ANSI B18.2.3.5M - 1979, The American Society of Mechanical Engineers, New York, 1979, p. 5.

30. Wilson, E. L., Taylor, R. L., Doherty, W. P., and Ghaboussi, J., "Incompatible Displacement Models," Numerical and Computer Methods in Structural Mechanics, edited by S. T. Fenves, N. Percone, A. R. Robinson, and W. C. Schnobrich, Academic Press, New York, 1973.

31. Goodier, J. N., "The Distribution of Load on the Threads of Screws," Journal of Applied Mechanics, Vol. 7, No. 1, March 1940, pp. A-10 to A-16.

32. Dann, R. T.,"How Much Preload For Fasteners?," Machine Design, Vol. 47, No. 20, 1975, pp 66-69.

33. Roark, R. J. and Young, W. C., Formulas for Stress and Strain, Fifth Edition, McGraw-Hill Book Company, New York, 1982, Table 24, pp. 332-338.

34. Bradley, T. L., "Stress Analysis for Thermal contact Resistance Across Bolted Joints," MS thesis, Massachusetts Institute of Technology, Mechanical Engineering Department, Cambridge, Massachusetts, August 1968.

35. Anonymous, "Exponential Least Squares," Plot 50 Statistics, Volume 4, Tektronix, Inc., Beaverton, Oregon, 1980, pp. 5-1 to 5-10. 


\section{Appendix A}

\section{Theoretical Radial Moment Equations}

The objective is to obtain the radial moment $M_{r}$ at $r=5 b$ due to an applied shear load at $r=a$ as a function of $a$, where $a$ is $\geq 5 b$. The plate geometry is shown in Figure 21 on page 54 . The equations are based on integration of the following fourthorder partial differential equation for bending of isotropic, axisymmetric plates of constant thicknesses with axisymmetric boundary conditions

$$
\frac{1 d}{r d r}\left\{r \frac{d}{d r}\left[\frac{1}{r} \frac{d}{d r}\left(r \frac{d y}{d r}\right)\right]\right\}=\frac{w}{D}
$$

where $y$ is the plate deflection, $w$ is the load per unit area, and $D$ is the plate rigidity constant given by

$$
D=\frac{E t^{3}}{12\left(1-v^{2}\right)}
$$

The radial bending line moment, $M_{r}$, is related to the deflection by 


$$
M_{r}=-D\left(\frac{d^{2} w}{d r^{2}}+\frac{v d w}{r d r}\right)
$$

Integrating of Eq. (A.1) thrice and substituting into Eq. (A.3) yields

$$
M_{r}=\theta_{b} \frac{D}{r} F_{7}+M_{\mathrm{rb}} F_{8}+Q_{b} r F_{9}-w r G_{9}
$$

where the coefficients $\theta_{b}, M_{\mathrm{rb}}, Q_{b}, \mathrm{~F}_{7}, \mathrm{~F}_{8}, \mathrm{~F}_{9}$, and $\mathrm{G}_{9}$ are given in Table 24, pp. 332-334, of Roark and Young [33]. $F_{7}, F_{8}$, and $F_{9}$, are functions of $r$ given by

$$
\begin{aligned}
& F_{7}=\frac{1}{2}\left(1-v^{2}\right)\left(\frac{r}{b}-\frac{b}{r}\right) \\
& F_{8}=\frac{1}{2}\left[1+v+(1-v)\left(\frac{b}{r}\right)^{2}\right] \\
& F_{9}=\frac{b}{r}\left\{\frac{1+v}{2} \ln \frac{r}{b}+\frac{1-v}{4}\left[1-\left(\frac{b}{r}\right)^{2}\right]\right\}
\end{aligned}
$$

The coefficient $G_{9}$ is also a function of $r$ but includes a multiplying singularity function which is zero for radii less than or equal to the radius of the applied shear load, $r_{0}$. For the problem of Figure 21 on page 54 in which the plate is loaded on the outer edge, all radii are less than or equal to the shear load radius and hence $G_{9}=0$.

Before applying the boundary conditions to obtain the remaining coefficients of Eq. (A.4), numerical values for $F_{7}, F_{8}$, and $F_{9}$ will be obtained. Inserting $r=5 b$ and $v=0.3$ into Eqs. (A.5) yields

$$
\begin{aligned}
& F_{7}=2.184 \\
& F_{8}=0.6640 \\
& F_{9}=0.2428
\end{aligned}
$$


Now the four different boundary condition cases of Figure 21 on page 54 will be used to obtain the remaining coefficients.

\section{Case A- Fixed Inner Edge, Free Outer Edge}

This corresponds to case 11 of Table 24 . The coefficients are given by

$$
\begin{aligned}
\theta_{b} & =0 \\
M_{\mathrm{rb}} & =\frac{-w a}{C_{8}}\left(\frac{a C_{9}}{b}-L_{9}\right) \\
Q_{b} & =\frac{w a}{b}
\end{aligned}
$$

The coefficients $\mathrm{C}_{8}, \mathrm{C}_{9}$, and $\mathrm{L}_{9}$ are defined as

$$
\begin{aligned}
& C_{8}=\frac{1}{2}\left[1+v+(1-v)\left(\frac{b}{a}\right)^{2}\right] \\
& C_{9}=\frac{b}{a}\left\{\frac{1+v}{2} \ln \frac{a}{b}+\frac{1-v}{4}\left[1-\left(\frac{b}{a}\right)^{2}\right]\right\} \\
& L_{9}=\frac{r_{0}}{a}\left\{\frac{1+v}{2} \ln \frac{a}{r_{0}}+\frac{1-v}{4}\left[1-\left(\frac{r_{0}}{a}\right)^{2}\right]\right\}
\end{aligned}
$$

Since the load is applied at the outer edge, $r_{0}=a$ and $\mathrm{L}_{9}=0$. Note that $\mathrm{C}_{8}$ and $\mathrm{C}_{9}$ are functions of $a$. Substituting $\mathrm{C}_{8}$ and $\mathrm{C}_{9}$ into Eq. (A.7) yields for $M_{\mathrm{rb}}$

$$
M_{\mathrm{rb}}=-2 w a \frac{\left\{\frac{1+v}{2} \ln \frac{a}{b}+\frac{1-v}{4}\left[1-\left(\frac{b}{a}\right)^{2}\right]\right\}}{\left[1+v+(1-v)\left(\frac{b}{a}\right)^{2}\right]}
$$

Substituting $v=0.3$ into Eq. (A.9) yields 


$$
M_{\mathrm{rb}}=-2 w a \frac{\left\{0.65 \ln \frac{a}{b}+0.175\left[1-\left(\frac{b}{a}\right)^{2}\right]\right\}}{\left[1.3+0.7\left(\frac{b}{a}\right)^{2}\right]}
$$

Substituting $M_{\mathrm{rb}}, r=5 b$, and the numerical values for $\mathrm{F}_{8}$ and $\mathrm{F}_{9}$ into Eq. (A.4) yields the radial moment as a function of $a$ for the fixed inner edge/free outer edge case

$$
M_{r}(r=5 b)=w a\left[1.214-1.328 \frac{\left\{0.65 \ln \frac{a}{b}+0.175\left[1-\left(\frac{b}{a}\right)^{2}\right]\right\}}{\left[1.3+0.7\left(\frac{b}{a}\right)^{2}\right]}\right]
$$

Note that $w$ is the shear load per unit of circumferential area. Since the shear load is applied at $r=a$, wa is the shear load/radian, a constant independent of the outer plate radius. The expression in large brackets in Eq. (A.11) is the nondimensional moment at $r=5 b$ which is plotted in Figure 21 on page 54 as a function of the outer radius $a$.

\section{Case B- Pinned Inner Edge, Free Outer Edge}

This case corresponds to case $1 \mathrm{k}$ of Table 24 . The coefficients are

$$
\begin{aligned}
M_{\mathrm{rb}} & =0 \\
\theta_{b} & =\frac{-w a^{3} C_{9}}{D b C_{7}} \\
Q_{b} & =\frac{w a}{b}
\end{aligned}
$$


where $C_{7}$ is given by

$$
C_{7}=\frac{1}{2}\left(1-v^{2}\right)\left(\frac{a}{b}-\frac{b}{a}\right)
$$

and $\mathrm{C}_{9}$ is defined in Eq. (A.8). Substituting for $\mathrm{C}_{7}$ and $\mathrm{C}_{9}$ into Eq. (A.12) yields

$$
\theta_{b}=\frac{-2 w a^{2}}{D\left(1-v^{2}\right)} \frac{\left\{\frac{1+v}{2} \ln \frac{a}{b}+\frac{1-v}{4}\left[1-\left(\frac{b}{a}\right)^{2}\right]\right\}}{\left(\frac{a}{b}-\frac{b}{a}\right)}
$$

Substituting $\theta_{b}, Q_{b}$, and $v=0.3$ into Eq. (A.4) with the numerical values for $F_{7}$ and $F_{9}$ yields the radial moment equation as a function of $a$ for this case.

$$
M_{r}(r=5 b)=w a\left[1.214-0.960 \frac{\left\{\frac{1+v}{2} \ln \frac{a}{b}+\frac{1-v}{4}\left[1-\left(\frac{b}{a}\right)^{2}\right]\right\}}{\left(1-\left(\frac{b}{a}\right)^{2}\right)}\right]
$$

\section{Case C- Fixed Inner Edge, Guided Outer Edge}

This case corresponds to case $1 \mathrm{j}$ of Table 24 . The coefficients are

$$
\begin{aligned}
\theta_{b} & =0 \\
M_{\mathrm{rb}} & =\frac{-w a}{C_{5}}\left(\frac{a C_{6}}{b}-L_{6}\right) \\
Q_{b} & =\frac{w a}{b}
\end{aligned}
$$


The coefficients $\mathrm{C}_{5}, \mathrm{C}_{6}$, and $\mathrm{L}_{6}$ are defined as

$$
\begin{aligned}
& C_{5}=\frac{1}{2}\left[1-\left(\frac{b}{a}\right)^{2}\right] \\
& C_{6}=\frac{b}{4 a}\left[\left(\frac{b}{a}\right)^{2}-1+2 \ln \frac{b}{a}\right] \\
& L_{6}=\frac{r_{0}}{4 a}\left[\left(\frac{r_{0}}{a}\right)^{2}-1+2 \ln \frac{a}{r_{0}}\right]
\end{aligned}
$$

Since the shear load is applied at $r_{0}=a, \mathrm{~L}_{6}=0$. Substituting into Eq. (A.16) yields for $M_{\mathrm{rb}}$

$$
M_{\mathrm{rb}}=\frac{-w a}{2} \frac{\left[\left(\frac{b}{a}\right)^{2}-1+2 \ln \frac{b}{a}\right]}{\left(1-\left(\frac{b}{a}\right)^{2}\right)}
$$

With the appropriate substitutions, Eq. (A.4) becomes

$$
M_{r}(r=5 b)=w a\left[1.214-0.3320 \frac{\left[\left(\frac{b}{a}\right)^{2}-1+2 \ln \frac{b}{a}\right]}{\left(1-\left(\frac{b}{a}\right)^{2}\right)}\right]
$$

\section{Case D- Pinned Inner Edge, Guided Outer Edge}

This case corresponds to case $1 \mathrm{i}$ of Table 24. The coefficients are 


$$
\begin{aligned}
M_{\mathrm{rb}} & =0 \\
\theta_{b} & =\frac{-w a^{3} C_{6}}{D b C_{4}} \\
Q_{b} & =\frac{w a}{b}
\end{aligned}
$$

where $\mathrm{C}_{4}$ is given by

$$
C_{4}=\frac{1}{2}\left[(1+v) \frac{b}{a}+(1-v) \frac{a}{b}\right]
$$

and $\mathrm{C}_{6}$ is defined in Eq. (A.17). After a little algebra and substitution, Eq. (A.4) yields

$$
M_{r}(r=5 b)=w a\left[1.214-0.2184 \frac{a}{b} \frac{\left[\left(\frac{b}{a}\right)^{2}-1+2 \ln \frac{b}{a}\right]}{\left[(1+v) \frac{b}{a}+(1-v) \frac{a}{b}\right]}\right]
$$

The nondimensional radial moments, $\frac{M_{r}}{w a}$, for these four cases are plotted in Figure 21 on page 54 as a function of the outer plate radius $a$.

The joint preload provides a natural means for scaling and nondimensionalizing the shear loads. However, for the case of the simulated applied radial moment, no natural quantity exists. This author chose to size the applied moment loads in relation to a reference moment obtained as follows. The reference moment is the radial moment at the inner edge of an annular plate fixed at the inner edge, free at the outer edge, and loaded with a shear load on the outer edge equal to the maximum applied shear load 
used in the bolted-joint finite element analysis. The plate has the same dimension as a joint member in the bolted joint studied here.

The moment equations are obtained from Table 24, case 11, of Roark and Young. The reference moment, $M_{R E F}$, is given by

$$
M_{\mathrm{REF}}=\frac{-w a^{2} C_{9}}{b \quad C_{8}}
$$

where $\mathrm{C}_{8}$ and $\mathrm{C}_{9}$ are defined in Eq. (A.8). Taking the joint member geometry a $=5 \mathrm{~b}$ and $v=0.3$, the coefficients have the values

$$
\begin{aligned}
& C_{8}=0.6640 \\
& C_{9}=0.2428
\end{aligned}
$$

The maximum applied shear load is given by

$$
P_{\max }=1.2 F_{i}=2 \pi a w
$$

Using Eq. (A.25) to obtain wa in terms of $F_{i}$ and substituting into Eq. (A.23) with the values from Eq. (A.24), one obtains

$$
M_{\mathrm{REF}}=0.349 F_{i}
$$

A joint preload of $39.3 \mathrm{kN}$ was used in the bolted-joint model. Hence, the reference radial line moment which was used to normalized the applied moment is

$$
M_{\mathrm{REF}}=13.72 \mathrm{kN}
$$




\section{Appendix B}

\section{Theoretical Deflection and Rotation of Joint}

\section{Member}

The objective is to determine the theoretical deflection and rotation of the joint member subject to a hypothetical fixed inner edge, free outer edge, and loaded with a pure shear load on the outer edge. Using the same notation as Figure 21 on page 54, the joint member has an inner radius $b$, and outer radius $a=5 b$, and a thickness $t=2.057 b$. These are the same geometric proportions used for the joint members in the finite element models. The outer edge deflection and rotation of the joint member is compared in Chapter 4 with the results of a linear finite element analysis of the joint member to assess the accuracy of the joint member mesh.

The joint member is analyzed as a thick plate with both bending and shear deflection. Table 24, case 11, and Table 25 of Roark and Young [33] are used to obtained the theoretical outer-edge deflection and rotation of the joint member. From Table 24, case 11 , the deflection and rotation at $r=a$ is given by 


$$
\begin{aligned}
& y_{a}^{b}=\frac{w a^{4}}{b D}\left(\frac{C_{2} C_{9}}{C_{8}}-C_{3}\right) \\
& \theta_{a}=\frac{w a^{3}}{b D}\left(\frac{C_{5} C_{9}}{C_{8}}-C_{6}\right)
\end{aligned}
$$

where the superscript $b$ denotes deflection due to bending, the subscript $a$ denotes the evaluation of the subscripted quantity at the outer edge, and $D$ is the plate rigidity constant given by

$$
D=\frac{E t^{3}}{12\left(1-v^{2}\right)}
$$

Substituting the expression for $D$ from Eq. (B.2) into Eq. (B.1) and rearranging yields

$$
\begin{aligned}
& y_{a}^{b}=K_{a}^{b}\left(\frac{w a}{E t}\right) \\
& \theta_{a}=C_{a}\left(\frac{w}{E t}\right)
\end{aligned}
$$

where

$$
\begin{aligned}
& K_{a}^{b}=\frac{12 a^{3}\left(1-v^{2}\right)}{b t^{2}}\left(\frac{C_{2} C_{9}}{C_{8}}-C_{3}\right) \\
& C_{a}=\frac{12 a^{3}\left(1-v^{2}\right)}{b t^{2}}\left(\frac{C_{5} C_{9}}{C_{8}}-C_{6}\right)
\end{aligned}
$$

The coefficients are defined in Table 24 as 


$$
\begin{aligned}
& C_{2}=\frac{1}{4}\left[1-\left(\frac{b}{a}\right)^{2}\left(1+2 \ln \frac{a}{b}\right)\right] \\
& C_{3}=\frac{1}{4 a}\left\{\left[\left(\frac{b}{a}\right)^{2}+1\right] \ln \frac{a}{b}+\left(\frac{b}{a}\right)^{2}-1\right\} \\
& C_{5}=\frac{1}{2}\left[1-\left(\frac{b}{a}\right)^{2}\right] \\
& C_{6}=\frac{b}{4 a}\left[\left(\frac{b}{a}\right)^{2}-1+2 \ln \frac{a}{b}\right] \\
& C_{8}=\frac{1}{2}\left[1+v+(1-v)\left(\frac{b}{a}\right)^{2}\right] \\
& C_{9}=\frac{b}{a}\left\{\frac{1+v}{2} \ln \frac{a}{b}+\frac{1-v}{4}\left[1-\left(\frac{b}{a}\right)^{2}\right]\right\}
\end{aligned}
$$

Substituting $a=5 b, t=2.057 b, v=0.3$ into Eqs. (B.4) and (B.5) yields

$$
\begin{aligned}
& C_{2}=0.2078 \\
& C_{3}=0.03569 \\
& C_{5}=0.4800 \\
& C_{6}=0.1129 \\
& C_{8}=0.6640 \\
& C_{9}=0.2428 \\
& K_{a}^{b}=13.00 \\
& C_{a}=20.20
\end{aligned}
$$

From Table 25 of Roark and Young, the deflection due to shear is given by

$$
y_{a}^{s}=k_{a}^{s} \frac{w a}{t G}
$$

where $G$ is the shear modulus and the coefficient $k_{a}^{s}$ for this case is given by

$$
k_{a}^{s}=1.2 \frac{r_{0}}{a} \ln \frac{r_{0}}{b}
$$

The shear modulus for the isotropic joint member is given by 


$$
G=\frac{E}{2(1+v)}
$$

Substituting Eqs. (B.8) and (B.9) into Eq. (B.7) yields

$$
y_{a}^{s}=K_{a}^{s}\left(\frac{w a}{E t}\right)
$$

where

$$
K_{a}^{s}=2(1+v) k_{a}^{s}=2.4(1+v) \frac{r_{0}}{a} \ln \frac{r_{0}}{b}
$$

Since the load is applied at $a, r_{0}=a=5 b$, and the shear deflection coefficient $K_{a}^{s}=5.021$. The total deflection due to bending and shear is

$$
\begin{aligned}
y_{a} & =y_{a}^{b}+y_{a}^{s} \\
y_{a} & =\frac{w a}{E t}\left[K_{a}^{b}+K_{a}^{s}\right] \\
y_{a} & =\frac{w a}{E t}(13.00+5.021) \\
& =18.02 \frac{w a}{E t}
\end{aligned}
$$

Note that shear deflection amounts to over $27 \%$ of the total deflection. From Eqs. (B.6) and (B.3) the rotation at the outer edge is given by

$$
\theta_{b}=20.20 \frac{w}{E t}=20.20 \frac{w a}{t a E}
$$

The quantity $w a$ is the shear load/radian applied at $r=a$ and is equal to the total applied shear load $\mathrm{P}$ divided by $2 \pi$. $\mathrm{P}$ was taken as $1.2 \mathrm{~F}_{\mathrm{i}}$, where $\mathrm{F}_{\mathrm{i}}=$ joint preload $=39.3$ kN. Hence, 


$$
\begin{aligned}
w a & =\frac{P}{2 \pi}=\frac{1.2 F_{i}}{2 \pi} \\
& =\frac{(1.2)(39.3)\left(10^{3}\right)}{(2)(3.142)} \\
& =7.51\left(10^{3}\right) \quad \mathrm{N} / \mathrm{rad}
\end{aligned}
$$

Taking $E=2.07\left(10^{11}\right) \mathrm{N} / \mathrm{m}^{2}, a=5 b, t=2.057 b$, and $b=1.0$ for simplicity, one obtains

$$
\begin{aligned}
& y_{a}=0.318\left(10^{-6}\right) \quad m \\
& \theta_{a}=0.711\left(10^{-7}\right) \mathrm{rad}
\end{aligned}
$$

The finite element results gave an average nodal deflection of the joint member edge of $0.312\left(10^{-6}\right) m$ yielding a percent error of +1.89 . Using the nodal radial displacements, an average edge rotation of $0.731\left(10^{-7}\right)$ rad was calculated. The percent error is -2.81 . 


\section{The vita has been removed from the scanned document}

